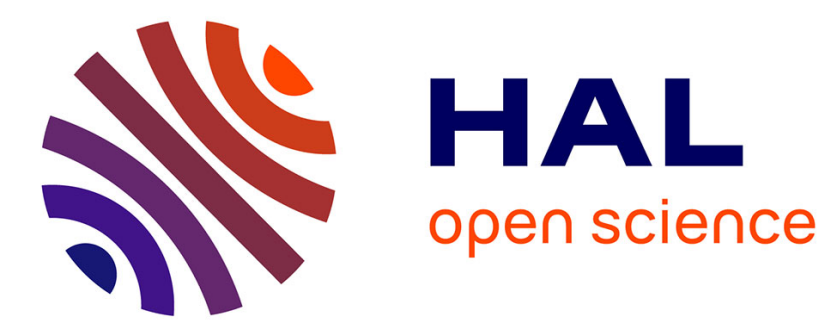

\title{
La chimiothérapie à domicile : complémentarité ou concurrence dans la stratégie des structures hospitalières
}

Valérie Buthion, Anouar Fanidi, Thierry Lagrange

\section{To cite this version:}

Valérie Buthion, Anouar Fanidi, Thierry Lagrange. La chimiothérapie à domicile : complémentarité ou concurrence dans la stratégie des structures hospitalières. 2010. hal-00605864

\section{HAL Id: hal-00605864 \\ https://hal.science/hal-00605864}

Submitted on 4 Jul 2011

HAL is a multi-disciplinary open access archive for the deposit and dissemination of scientific research documents, whether they are published or not. The documents may come from teaching and research institutions in France or abroad, or from public or private research centers.
L'archive ouverte pluridisciplinaire HAL, est destinée au dépôt et à la diffusion de documents scientifiques de niveau recherche, publiés ou non, émanant des établissements d'enseignement et de recherche français ou étrangers, des laboratoires publics ou privés. 

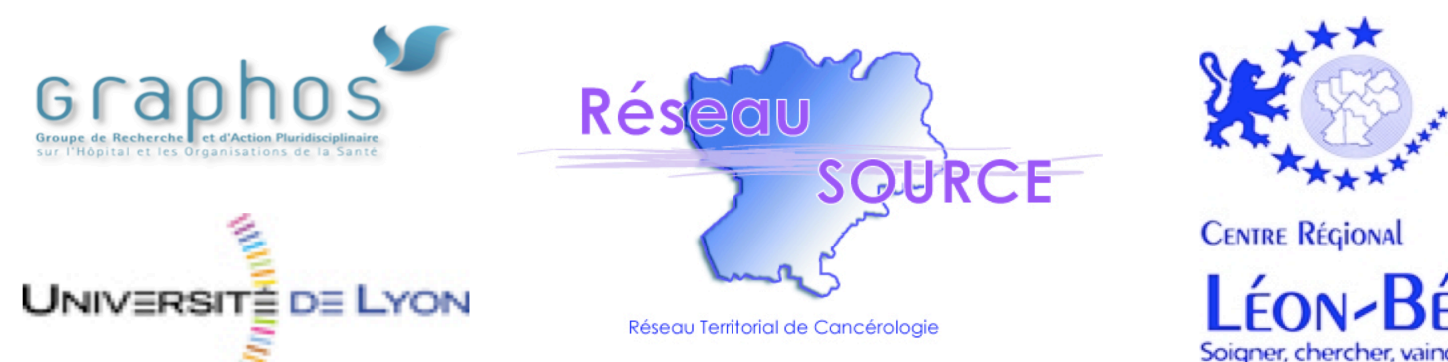

Centre Récional

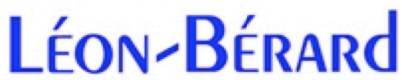

Soigner, chercher, vaincre. Ensemble

\title{
La chimiothérapie à domicile : complémentarité ou concurrence dans la stratégie des structures hospitalières
}

\section{Rapport de Recherche}

\author{
Juillet 2010 \\ ETUDE REALISEÉ AVEC LA PARTICIPATION FINANCIERE DE LA REGION RHONE-ALPES \\ Projet OSAD - Cluster GOSPI
}

Etude réalisée et dirigee par Valérie Buthion (1), avec la participation de Anouar Fanidi (2) et Thierry Lagrange (3)

(1) Maitre de Conferences, universite lyon 2, Graphos (Groupe de Recherche Appliquee Pluridisciplinaire sur l'Hopital et les Organisations de Santé), Chercheur associe a l'axe Economie de la Sante du Centre Léon Berard

(2) Infirmier Diplôme d’Etat, Etudinnt en Master Sante et Population, Universite Lyon 1, Centre Léon Berard

(3) Coordonateur du Reseau Territorial de Cancerologie Source 
Page 2 sur 122 


\section{Table des matières}

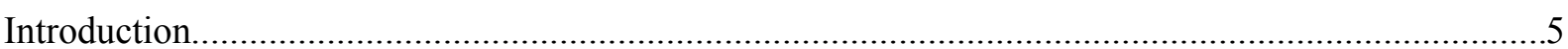

Première Partie : Méthodologie utilisée pour l'analyse organisationnelle...........................................8

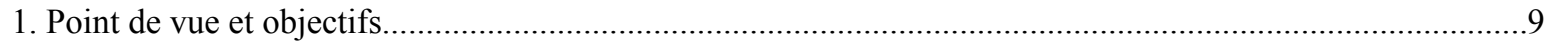

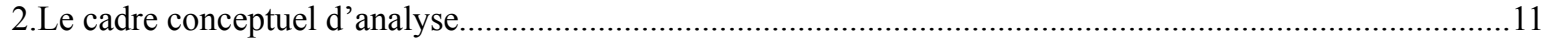

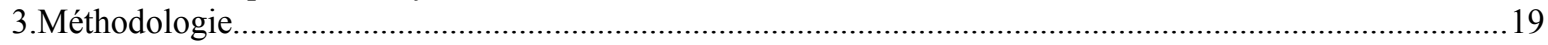

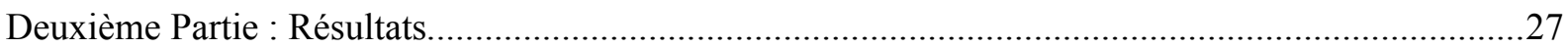

1.Synthèse des opinions : croyances, contradictions, interrogations..............................................................27

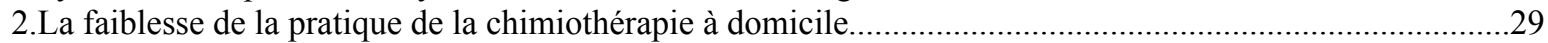

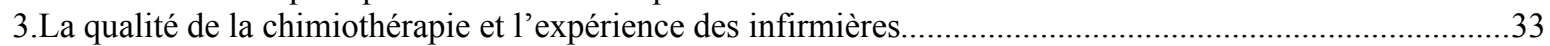

4.L'activité de chimiothérapie à domicile : des infirmières disponibles...........................................................34

5.La question du coût pour l'assurance maladie : une question difficile à trancher..............................................35

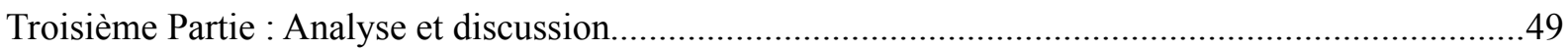

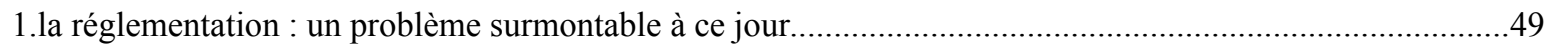

2.Des professionnels de santé disponibles en dehors de l'hôpital...................................................................50

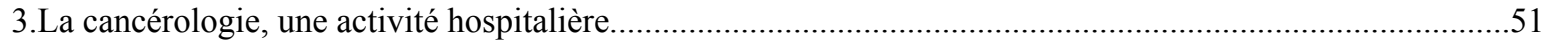

4.Un financement désormais dépendant de l'activité mesurée........................................................................52

5.La chimiothérapie à domicile : une solution avec peu d'avenir ?.............................................................53

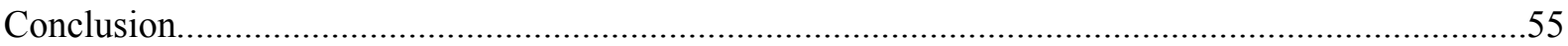

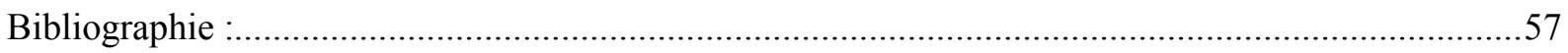

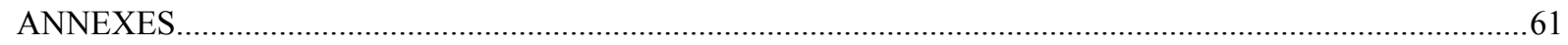


Page 4 sur 122 


\section{Introduction}

Le traitement du cancer requiert l'usage de molécules de chimiothérapie, administrées en plusieurs séquences successives, à ce jour principalement par voie intraveineuse. Il nécessite l'intervention d'un personnel soignant qualifié et est effectué principalement dans les services de jour des hôpitaux et des cliniques qui ont organisé en 2009 près de deux millions de séances. Dans son rapport sur la situation de la chimiothérapie des cancers, l'Institut National du Cancer souligne l'accroissement du volume des chimiothérapies liées à la prévalence du cancer dans la population qui est en augmentation et du plus grand usage qui est fait des molécules de chimiothérapie. La part de la chimiothérapie dans le coût des séjours d'hospitalisation pour cancer s'élevait en 1,58 milliards d' $€$ en 2008, soit près d' $1 / 3$ des coûts de traitements des cancers. Plus de $60 \%$ de ces dépenses sont liées aux molécules innovantes et « onéreuses ». Si le rapport en appelle à un contrôle plus important du « bon usage » des ces molécules, le rapport n'aborde pas la question du coût de leur administration. (INCA, 2009).

L'idée de réaliser les chimiothérapies au domicile des patients, administrées par les infirmières libérales de ville, s'est développée dès les années 90 suite à l'effet combiné de l'augmentation des cancers qui saturaient les plateaux techniques de cancérologie et d'une volonté d'externaliser certaines prestations hospitalières pour réduire les coûts de prise en charge. La mesure $\mathrm{N}^{\circ} 41$ du Plan Cancer 2003 souhaitait «faciliter la chimiothérapie à domicile et plus généralement les soins à domicile» (DGS, 70 mesures Plan Cancer). Côté dépenses de transport des patients, les coûts ont représenté 2,3 mds en 2008 sur quelques 170 Mds d' $€$ de dépenses de la branche maladie de la sécurité sociale, soit environ 1,35\% des dépenses. La cancérologie tient une bonne part de ces dépenses.

Pour assurer la sécurité de cette prestation, l'ANAES ${ }^{1}$ a publié des recommandations sur les critères d'éligibilité des patients aux chimiothérapies anticancéreuses à domicile, à la demande de la $\operatorname{DHOS}^{2}$ (HAS 2005). Dès 2006, les possibilités ont été restreintes à la réalisation dans deux cadres organisationnels bien précis : l'Hospitalisation à Domicile ou les Réseaux de Soins. La première séance, ou toute séance comportant une nouvelle molécule, doit être faite à l'hôpital pour vérifier l'effet toxique immédiat qu'elle peut avoir sur les patients. La molécule est préparée de façon centralisée par une Pharmacie Hospitalière, puis

\footnotetext{
${ }^{1}$ Agence Nationale d'Accréditation et d'Evaluation en Santé

${ }^{2}$ Direction de l'Hospitalisation et de l'Organisation des soins
} 
« revendue » pour être administrée à domicile. Les molécules concernées sont inscrites sur une liste spécifique, dite liste de rétrocession.

Aujourd'hui, de nombreuses molécules de chimiothérapie peuvent être administrées dans le cadre d'une structure d'Hospitalisation à Domicile (HAD) ou à domicile par des libéraux coordonnés par un réseau de soins. Or, en 2009, la chimiothérapie à domicile représente moins de $1 \%$ de près de 2 millions de séances ambulatoires organisées chaque année en France. L'objet de ce rapport est de présenter les résultats d'une étude sur l'organisation de la chimiothérapie ambulatoire et de ses alternatives à domicile, que nous avons mené dans le cadre d'un projet portant sur l'Organisation des Soins à Domicile (Projet OSAD, financé par la région Rhône Alpes). Cette étude investigue les données relatives aux coûts des alternatives, mais également les stratégies des différents acteurs de soins au regard de cette prestation. Partant d'une comparaison approfondie de la structure de coût et de financement de ce soin par l'assurance maladie, nous avons chercher à élucider la faiblesse de la chimiothérapie à domicile.

Se rendre à l'hôpital peut permettre au patient de bénéficier d'autres soins dans la même journée (radiothérapie, examen, autres soins). Cependant, cela ne représente qu'une partie des séjours. Dans les autres cas, le patient vient seulement recevoir sa ou ses molécules de chimiothérapie.

Les études économiques comparatives n'ont pas jusqu'à présent montré de façon probante la supériorité économique de l'une ou de l'autre solution pour l'assurance maladie. Des tarifications mouvantes, des informations incomplètes ou mal renseignées, n'ont pas permis d'avoir une vision claire de la situation. Pourtant, les croyances des acteurs de soins sont assez tranchées. Ceux qui défendent les soins à domicile sont persuadés que l'administration à domicile coûte moins cher qu'à l'hôpital, alors que les détracteurs pensent assez souvent le contraire.

Disposant d'un matériau riche d'entretiens préalablement réalisés auprès d'acteurs des soins à domicile, nous avons exploité en seconde intention plusieurs études antérieures pour en dégager les opinions dominantes chez les différents acteurs, opinions qui font apparaître des croyances dominantes ou des contradictions qu'il convient de documenter. Nous avons réalisé une étude systématique de la littérature, des données statistiques disponibles, des données d'un réseau territorial de cancérologie, le réseau Source, réalisé de façon complémentaire une étude par questionnaire auprès des infirmières libérales. Nous avons également collecté des 
données par entretien et questionnaire auprès des HAD.

Nous présenterons, en mettant en perspective des données financières, des éléments quantitatifs et des éléments qualitatifs qui permettent d'expliquer l'état actuel de l'offre de soins sur cette prestation. Cela nous permet de montrer que ce ne sont pas des raisons financières, mais les modèles d'activité des hôpitaux, qui freinent le développement de la chimiothérapie à l'extérieur de l'hôpital.

La première partie présentera la méthodologie de l'étude. La seconde partie exposera l'essentiels des résultats, qui sont présentés dans leur intégralité en annexe. La troisième partie présentera la synthèse, l'analyse et la discussion. 
Page 8 sur 122 


\section{Première Partie : Méthodologie utilisée pour l'analyse organisationnelle}

Nous présentons dans cette partie le point de vue retenu, le cadre conceptuel que nous avons utilisé pour l'étude, les considérations méthodologiques générales, les modalités de recueil et d'exploitation des données. Les choix méthodologiques que nous avons faits pour développer cette étude s'inscrivent dans les méthodes de Recherche Action-Intervention, pratiquées depuis quasiment un siècle et qui se sont beaucoup structurées dans les années 1980-90 (Whyte, 1989, 1995, Argyris et al 1985). Elles reposent sur une investigation dans un contexte social où nous cherchons à doser deux postures pour la recherche : une posture pour partie « réflexive» rendant compte du questionnement émanant des acteurs et une posture pour partie « dialectique », introduisant la contradiction avec les acteurs avec le dessein de faire émerger de nouvelles solutions. Le but de la Recherche Action-Intervention est ouvertement de faciliter la transformation de l'environnement (Oja, Shulman, 1989).

Pour cela, la méthode d'investigation doit être capable de rendre compte de la « généalogie de l'action collective » étudiée et être à même d'apporter un « soutien à l'invention de nouvelles formes » (Hatchuel, 2008) Nous allons donc nous intéresser à la fois à ce qui se passe au présent, mais également à la manière dont les organisations étudiées se projètent dans le futur.

Dans notre cas, nous n'étudions pas une organisation particulière dispensant des soins, mais

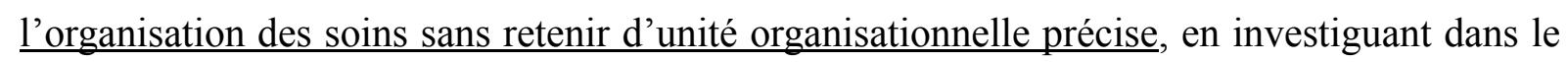
détail les situations de concurrence ou de complémentarité des différentes organisations (ou groupes organisés) ou des différents acteurs (ou individus). La prise en charge d'un ou plusieurs épisodes de soins est parfois à ce point complexe que ce sont souvent plusieurs organisations différentes qui vont intervenir, avec plusieurs personnes de statuts différents,

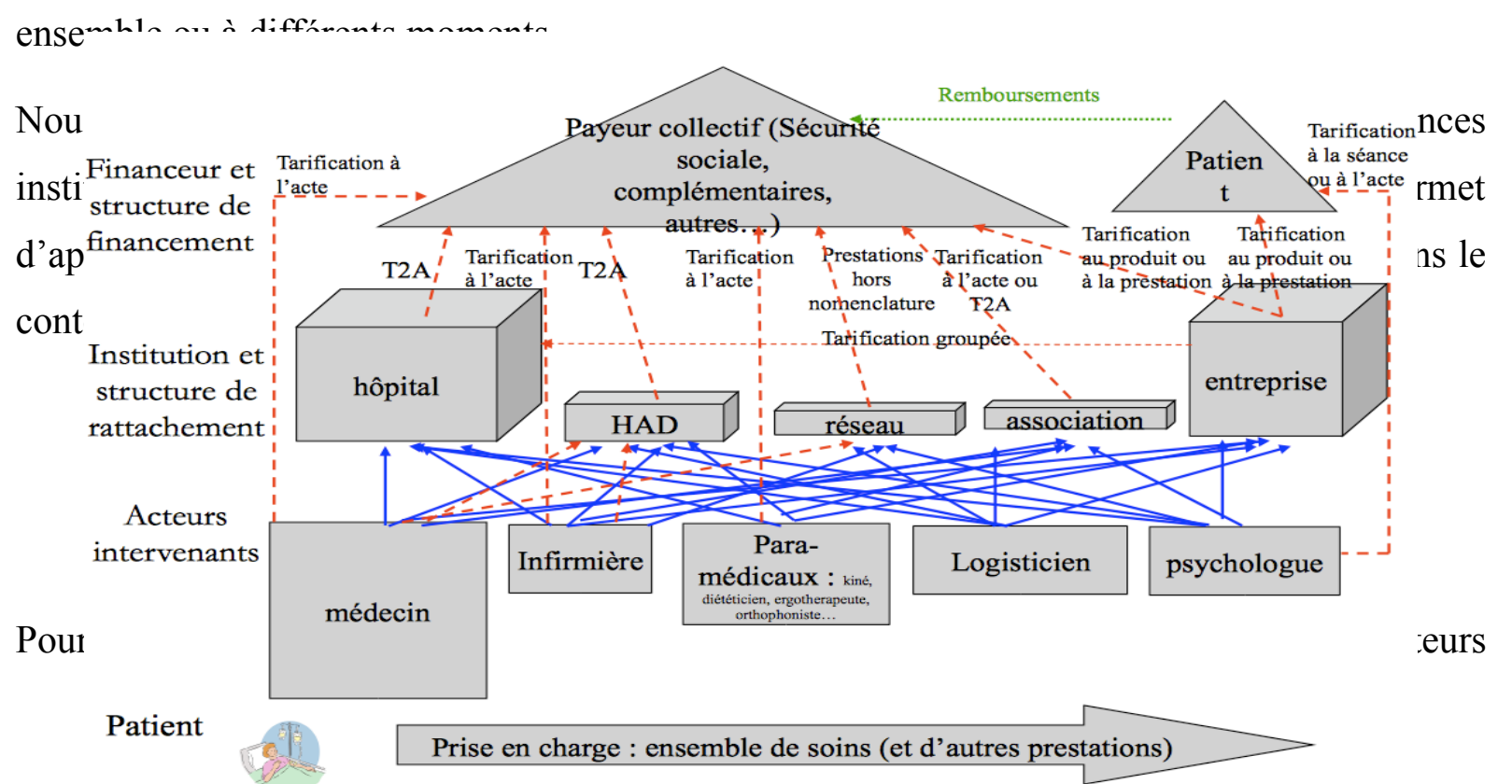


et les institutions représentés n'ont pas le même poids, aussi sont-ils représentés symboliquement avec des tailles légèrement différentes. Du point de vue de la prescription, les médecins sont la catégorie d'acteur dominante, et comme, dans le cas de la cancérologie, ils sont majoritairement hospitaliers, l'hôpital est l'institution dominante. Les entreprises du secteur, particulièrement celle du médicament, sont également en position de force grâce à leur poids économique. Les autres acteurs jouent un rôle faible, non qu'ils ne soient pas importants. Par exemple, les infirmières sont un maillon fondamental de la chaîne de soins , mais ont peu d'influence dans la conception des parcours de soins.

L'abondance des flèches illustre des flux d'allocation de ressources passant par des circuits assez complexes, qui sont parfois aussi illisibles pour les acteurs de soins que le schéma qui les représentent ici. Les mêmes acteurs ont parfois plusieurs façons de pouvoir se faire rémunérer pour leur travail, et cela aura fatalement des incidences sur leur comportement.

\section{Point de vue et objectifs}

A l'origine interrogé sur le coût comparé des différents modes d'administration de la chimiothérapie, dans un contexte ou chaque acteur n'a qu'une vue partielle des coûts réellement supportés par le système de soin, le point de vue retenus pour cette étude a été celui de 1'Assurance Maladie. Nous souhaitons cependant, au-delà des considérations financières, mettre en lumière l'agencement de ces organisations dispensant des soins de santé dans la prise en charge d'un patient pour un soin ou un parcours de soin. L'analyse de l'organisation des parcours de soins que nous tentons de construire cherche à étudier de façon systématique les conditions dans lesquelles les acteurs vont s'organiser. Dans un parcours de soins composé d'un ou de plusieurs épisodes (séjour hospitalier et/ou soins ambulatoires), pris en charge par différents acteurs appartenant à différentes organisations, plusieurs solutions vont émerger. Quelles sont les combinaisons qui semblent les plus appropriées, selon quel(s) critère(s) ou quel point de vue et comment pourraient-elles fonctionner dans l'avenir. Notre analyse ne se focalise pas seulement sur les données économiques, mais prend en compte d'autres paramètres qui conduisent les uns et les autres à faire des arbitrages et de les imposer de façon plus ou moins contraignantes au système de soins.

L'analyse organisationnelle a pour but de documenter le comment et le pourquoi de la formation de l'offre de soin à un instant donné. La tutelle et l'Assurance Maladie utilisent classiquement deux leviers pour contraindre ou inciter les acteurs à faire ou ne pas faire : la réglementation et l'allocation de ressources. Cependant, les expériences passées ont montré 
que ces leviers étaient à eux seuls insuffisants pour expliquer la formation de l'offre. Ainsi, certains textes et financements visant à encourager le développement de telle ou telle forme de prise en charge : les réseaux de soins, les Hospitalisations à Domicile, ou plus récemment les maisons médicales pluridisciplinaires, n’ont pas eu le succès escompté. Nous cherchons donc à étudier, à l'intérieur même du système de soins, les motivations, résistances, intérêts, jeux d'acteurs qui motivent l'offre de soins.

Le système de soin dans son ensemble intègre les différentes contraintes de l'environnement et amène certaines réponses qui sont plus ou moins satisfaisantes.

- Quels sont les dysfonctionnements observés dans la conception ou la mise en œuvre de ces réponses?

- Comment cette organisation répond (ou n'arrive pas à répondre) aux besoins de santé des populations?

- Quelles ressources sont mobilisées?

- Dans quelles conditions cette offre est susceptible de se modifier à court (dans l'année ou les deux années qui viennent) et à moyen terme (à plus de deux ans, de façon prospective) ?

\section{Le cadre conceptuel d'analyse}

Le cadre d'analyse présenté ci-après est un aboutissement des travaux de recherche que nous avons engagé dans le domaine de l'organisation des soins. Il a été finalisé et appliqué pour la première fois dans le cadre d'une étude sur l'organisation de la transfusion sanguine ambulatoire (Buthion, 2009). Il repose sur une modélisation de deux ensembles inventoriant des caractéristiques dont nous jugeons pertinent d'observer les interactions : le modèle « patient » et le modèle « organisation ». Ces deux modèles, et les espaces de décisions qui les portent, vont produire opportunités et contraintes et entrer en interaction dans la production des soins. L'observation des paramètres qui sont actionnés dans l'organisation du soin observer permet d'expliquer l'état de l'organisation actuelle et de révéler les freins à lever ou les forces motrices à actionner pour la modifier de façon significative.

\subsection{Le modèle Patient}

Tout d'abord, il convient de préciser que nous parlons bien de patient, qui souffre et qui nécessite des soins médicaux, et que cette méthode n'a pas été développée pour étudier le bon 
fonctionnement des dispositifs de prévention destinés à la population en général. Les attributs du patient qui nous intéressent sont les caractéristiques listées ci-dessous qui vont constituer autant de contraintes pour l'organisation des soins (Figure 2).

Nous regroupons ces caractéristiques en trois sous-modèles distincts.

- Le modèle clinique vise à identifier les contraintes liées à la pathologie. L'état de santé est, bien entendu, central, et structurera les exigences quant à la complexité des plateaux techniques nécessaires. Aussi, ce modèle est principalement construit sur l'identification de l'influence de l'état de santé, des facteurs pronostics et de l'âge des patients, qui sont les caractéristiques prises en compte dans l'élaboration des programmes thérapeutiques. Nous retiendrons donc dans ce modèle les seuls paramètres cliniques expliquant les décisions prises pour le patient, afin d'en analyser correctement les contraintes quant au lieu et au mode de prise en charge. Il ne nous appartient pas, bien entendu, d'évaluer la pertinence des décisions médicales. En effet, pour déterminer la pertinence d'une contrainte sur le plan médical, nous nous référerons aux avis d'experts, à la littérature médicale et aux recommandations. Par contre, le système de soins doit répondre à des contraintes d'efficacité clinique, par exemple dans le cas de la transfusion de globule rouge de permettre une amélioration de l'état de fatigue générale du patient.

Figure 2: Le modèle « patient »

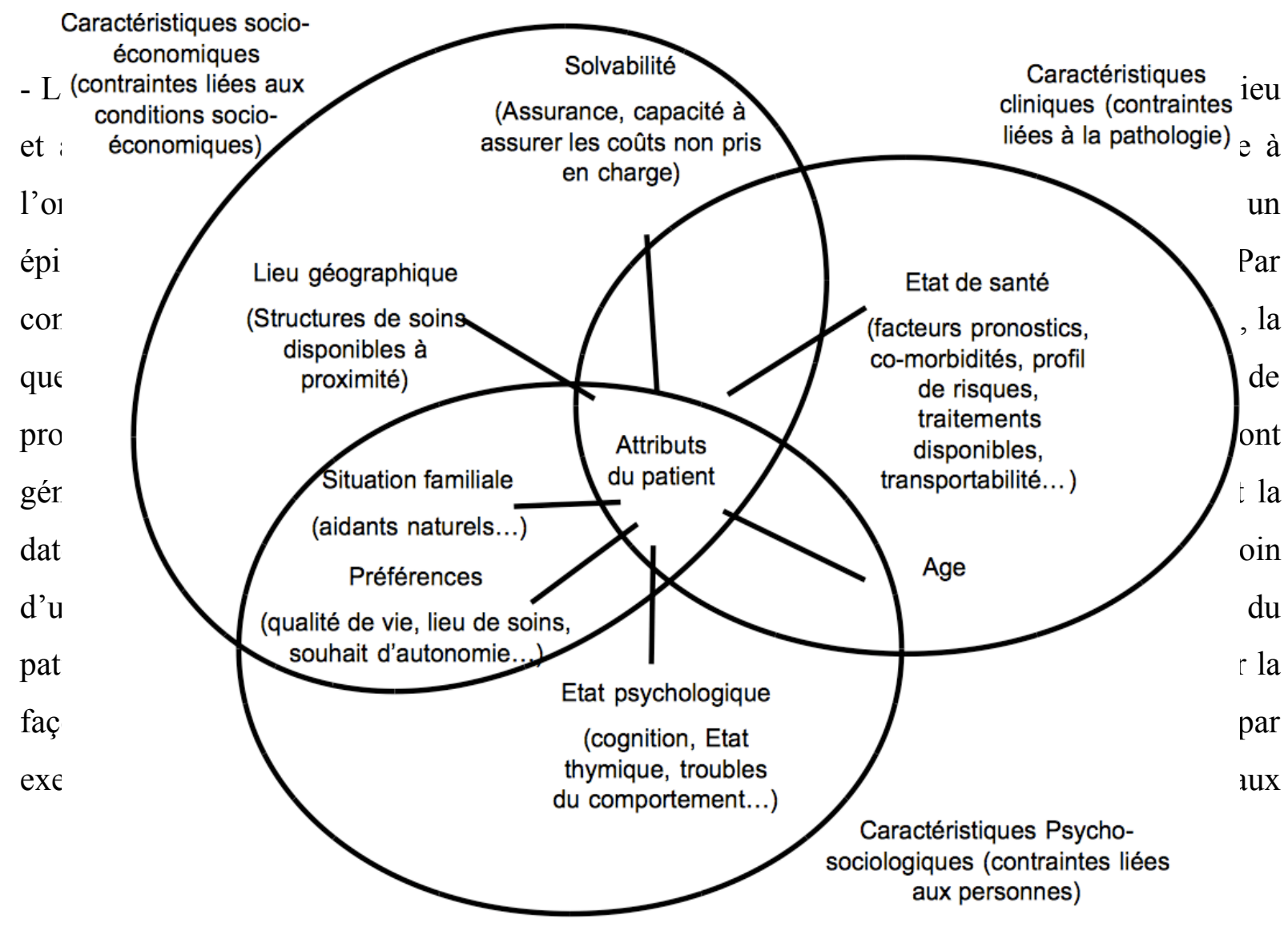


contraintes financières des patients. Il ne s'agit pas dans notre méthode d'investigation de se prononcer sur la pertinence du modèle social, mais d'identifier, parmi les réponses existantes, celle qui est adaptée au déroulement du parcours de soins.

- Le modèle psycho-social s'intéresse aux contraintes créées par les conditions psychologiques dans lesquelles se trouvent le patient et ses accompagnants. Les problèmes cognitifs de certains patients âgés qui ont des incidences sur l'observance de la prise de médicaments ou la situation de détresse psychologique que créent les situations de fin de vie peuvent nécessiter par exemple un accompagnement psychologique spécifique sans lequel certains soins ne peuvent être pris en charge. Les patients peuvent également exprimer plus ou moins ouvertement leurs préférences, pour tel type de traitement, pour tel lieu de soin, voire refuser d'aller dans tel ou tel établissement. La loi de 2002 leur a à cet égard donné quelques droits supplémentaires. Tout comme nous l'évoquions à propos du modèle socio-économique, il ne nous appartient pas de décider dans quelle proportion le système de soin doit assumer ces contraintes, mais nous ne pouvons pas les ignorer a priori.

Les trois sous-modèles sont perméables et sont présentés en tant que tel dans le schéma. De nombreuses études ont montré que l'état de santé était influencé par le niveau de revenu (Jusot, 2003) et l'état psychologique du patient. L'OMS définit d'ailleurs que « La santé est un état de complet bien être physique, mental et social et ne consiste pas seulement en une absence de maladie ou d'infirmité." Cependant, il n'est pas dans les attributions actuelles des organisations dispensant des soins de répondre à toutes ces problématiques avec des soins médicaux. Le champ d'application principal des systèmes de soins que nous étudions est de traiter de la maladie et de sa résorption ou de son accompagnement. Aussi, dans le contexte de l'étude des soins médicaux et infirmiers, le modèle clinique est celui qui imposera le plus de contraintes. Dans l'approche de la prise en charge d'une pathologie, dont les soins peuvent se dérouler sur plusieurs années, voire sur le reste de l'existence de l'individu dans le cas des pathologies chroniques, l'approche doit être élargie aux autres modèles. La maladie d'Alzheimer est un exemple où il est impossible de restreindre l'approche aux paramètres de l'état clinique.

Ce modèle opère selon nos travaux actuels sur au moins 4 espaces. Le déploiement de ses espaces (figure 3) permet de montrer comment les paramètres ou attributs du patient sont pris en compte et conduits à s'influencer les uns les autres. La mise en cuvre d'une chimiothérapie chez un patient atteint de cancer peut avoir à tenir compte notamment, au-delà des contraintes strictement médicales, du lieu d'habitation et de l'éloignement entre le patient 
et le centre de soins, de la présence ou non d'aidant pour les phases inter cures ou de ses choix personnels.

Pendant longtemps condamné à subir les choix du corps médical, quant au traitement ou au lieu de soins, le patient, parfois appelé consommateur de soins pour coller aux modèles microéconomiques, est de plus en plus souvent sollicité pour participer aux décisions de santé qui le concerne. Cette tendance, qui relève en France du mouvement relatif à la démocratie sanitaire, se développe depuis le début des années 90. Des travaux menés au niveau international comme au niveau français montrent une démarche rigoureuse visant à surmonter les difficultés à connaître les préférences de patients qui, plongés dans la situation stressante de la maladie, ne sont pas en position de prendre des décisions de façon sereine. (Coulter et al, 1999, Charles et al, 1997, 1999, Moumjid et al, 2009). Il convient pour ces auteurs d'aider les patients à révéler leurs préférences. Dans le domaine de la cancérologie, où les fins de vie peuvent être longues et douloureuses, cet espace des «décisions personnels » va voir se développer des réflexions et des revendications autour des thèmes de l'acharnement thérapeutique, de la fin de vie, de l'euthanasie. La psychologie, la philosophie, le droit notamment ont alors toute leur place auprès de la dimension médicale, et bien entendu des impacts importants quant à l'organisation des soins.

Figure 3 : Déploiement des attributs du modèle "patient" dans une cartographie des espaces de décision

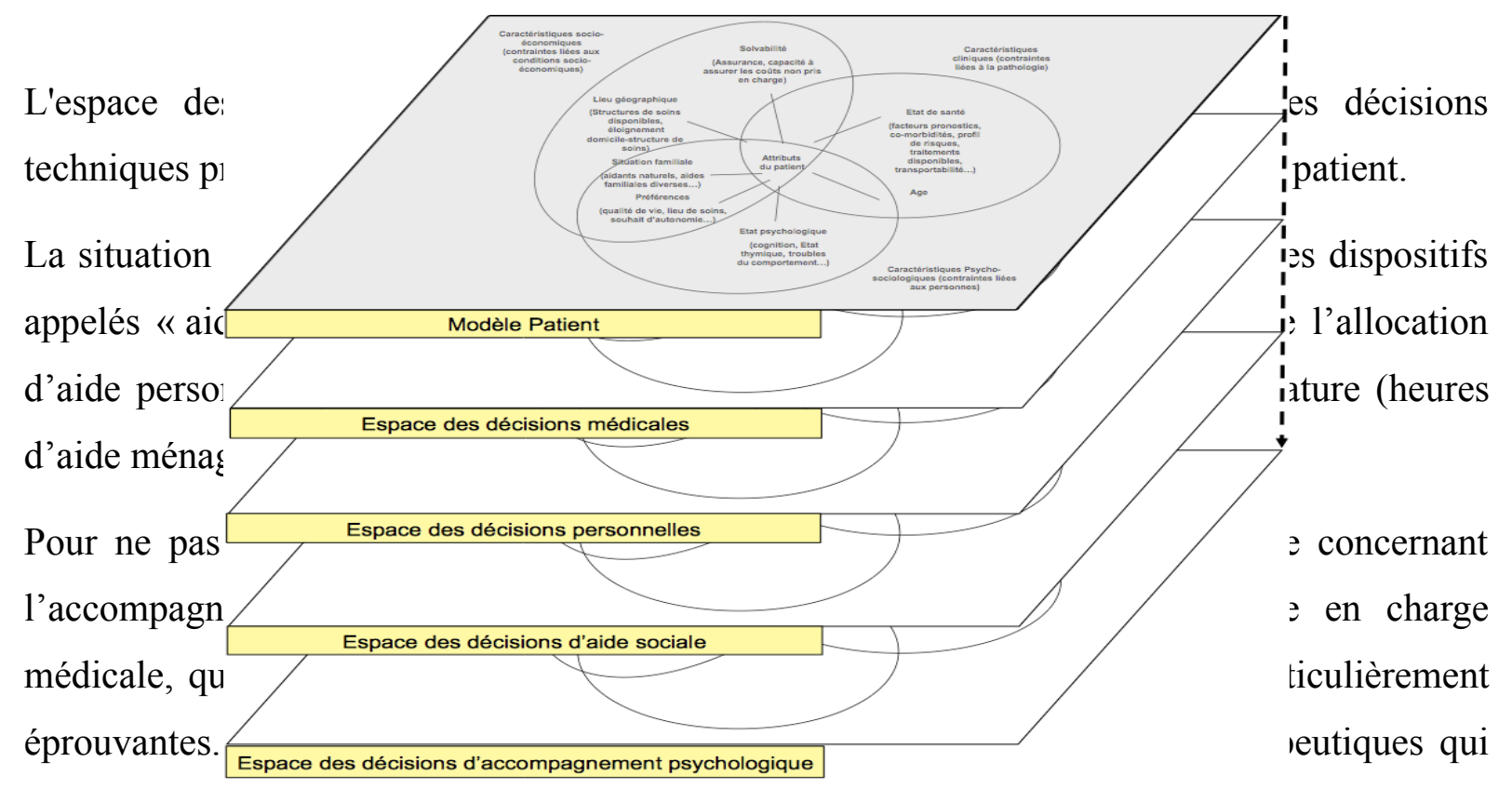

relève de la psychiatrie, l'espace de décision qui est mis en œuvre est celui de décisions médicales. 
Si dans le contexte du système de soins, conçu par nature pour prendre en charge la maladie, l'espace des décisions médicales est logiquement l'espace dominant des décisions définissant la prise en charge, d'autres espaces s'invitent donc de plus en plus fréquemment dans la décision d'organisation des soins. Un espace de décision n'est pas assimilable à une spécialité, mais à une logique de décision. De même, un espace n'est pas assimilable à une personne. Plusieurs personnes peuvent intervenir pour former les décisions dans un espace, comme par exemple la famille dans celui des décisions personnelles.

\subsection{Le modèle « Organisation »}

Le modèle d'analyse que nous avons construit pour mettre ces composantes en perspective est très directement adapté de l'interaction des contraintes économiques et des contraintes stratégiques caractérisant le «business model» tel qu'il est représenté par Henri Bouquin (Bouquin, 2008). Une organisation qui intervient dans le monde de la santé est une structure plus ou moins importante (un hôpital, une clinique, une structure associative à but non lucratif, un réseau, un spécialiste installé en libéral...). Cette organisation poursuit des objectifs plus ou moins hiérarchisés, fixés par les acteurs qui la gouvernent (modèle stratégique). Pour atteindre ces buts, les acteurs doivent se procurer des ressources (modèle économique). Le modèle de fonctionnement est donc le résultat de la combinaison du modèle stratégique et du modèle économique (figure 4). Le modèle « organisation » rassemble les caractéristiques relatives aux intentions stratégiques des acteurs (ce qu'ils souhaitent faire et comment ils le font compte tenu des contraintes de leur environnement immédiat) et aux mécanismes qui leur permettent de se procurer des ressources.

La stratégie doit être pensée comme un ensemble complexe qui, pour exister, nécessite de dépasser la seule équation du profit (Martinet 2006). Une organisation qui ne se définit que par l'intermédiaire de son modèle des flux financiers est une organisation qui fait preuve d'un opportunisme à court terme et se contente de répartir des revenus financiers entre ses différents membres. Chacune des activités développées doit alors dégager un excédent de ressources sur les dépenses qu'elle induit, et il n'y a pas de raison d'utiliser les excédents pour autre chose que de rémunérer les actionnaires (profits), adhérents (baisse des cotisations ou prestations en nature), ou autres parties prenantes (augmentation des salaires, avantages en nature, reversement des excédents au budget de l'Etat). Pas question par exemple de développer des innovations qui ne pourraient être immédiatement rentabilisées. On se contentera donc de copier ce que les autres font, et on les laissera tester les nouveaux 
concepts. À l'opposé, une organisation qui ne se penserait que stratégique, sans se préoccuper de son modèle économique, se retrouverait rapidement avec une somme de projets émiettés pour lesquels elle n'aurait pas les ressources financières suffisantes. La capacité de survie d'une organisation dans le temps dépend donc de la capacité de ses acteurs à maintenir un équilibre sans cesse renouvelé entre l'un et l'autre de ces modèles.

\section{Figure 4 : Le modèle "Organisation"}

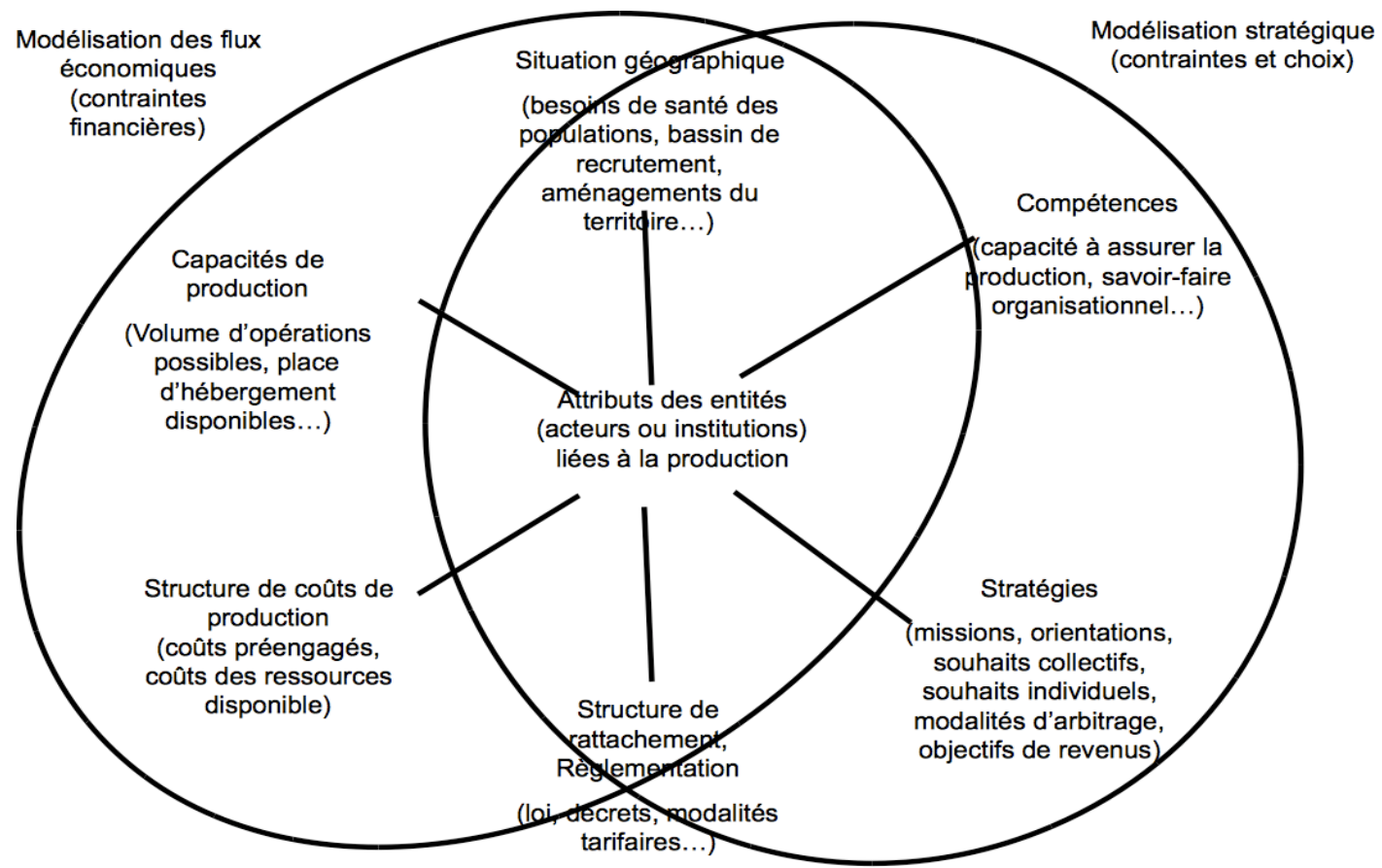

De la même manière, le modèle « organisation » peut être observé à travers au moins quatre espaces de décision intervenant dans la composition de l'offre de soins (figure 5). Plusieurs espaces de décision vont alors intervenir, où chaque acteur animera les contraintes de son propre modèle d'activité.

Le premier est l'espace des décisions des individus qui sont porteurs des compétences nécessaires au fonctionnement du système de soins. La spécialisation des compétences et le cas échéant leur rareté leur confère une capacité à accepter, refuser ou construire les conditions d'exercice de leur profession. 
Figure 5: déploiement des attributs du modèle « organisation » dans une perspective de cartographie des décisions

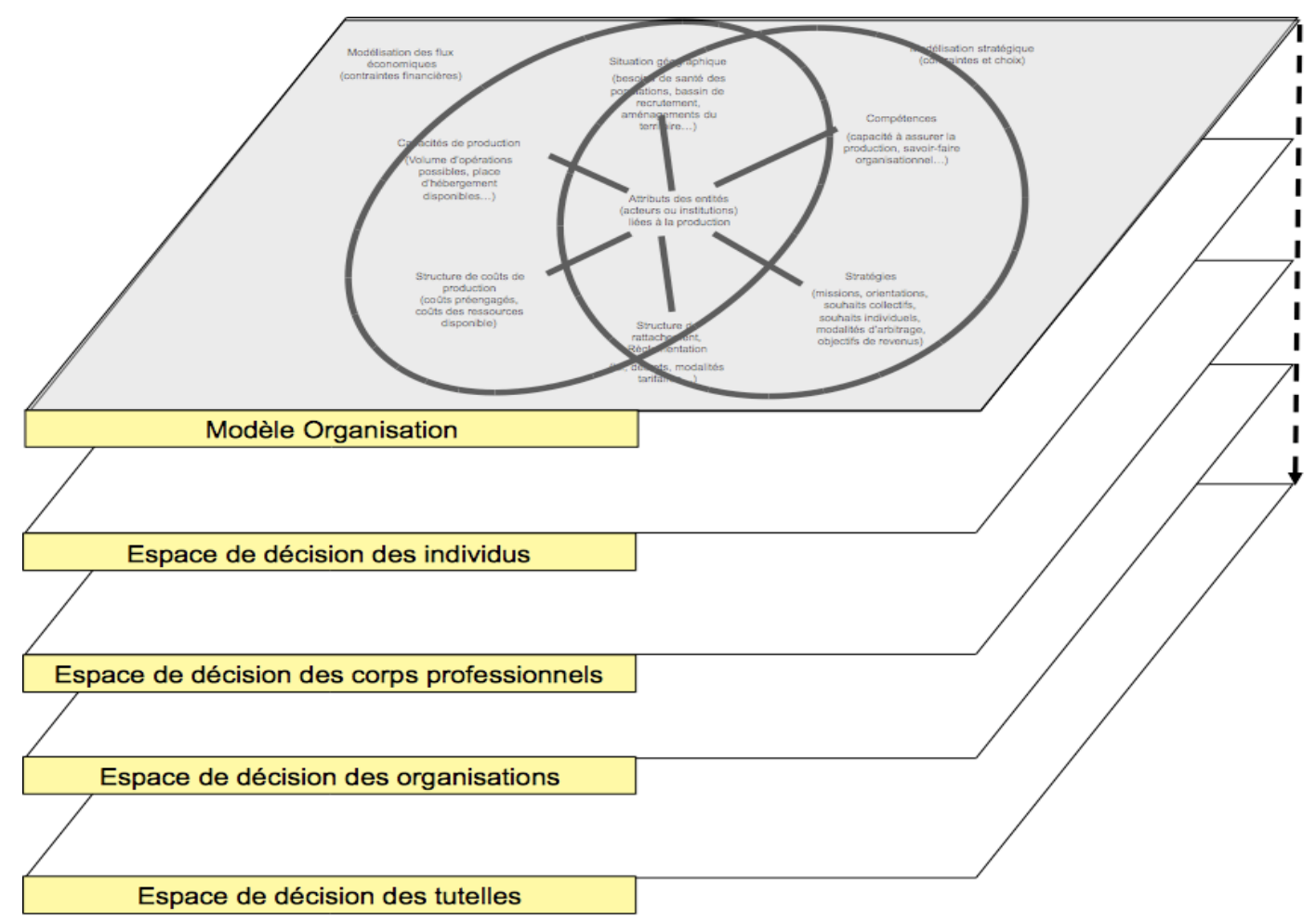

Le second espace est celui des corps professionnels constitués dans le monde de la santé, régis par des ordres (médecins, maintenant infirmiers), des associations de spécialistes, dans un espace qui, si on lui applique le modèle de Mintzberg, apparaît fortement structuré par une standardisation des compétences et une reconnaissance de ces compétences par les pairs (Mintzberg, 1982).

Le troisième espace est celui des organisations agissant dans le domaine de la production de soins : les hôpitaux, cliniques, centres de soins, cabinets médicaux, pharmacies d'officine, industriels du matériel ou du médicament, etc...

Enfin, le dernier espace est celui des tutelles du système de santé, qui y déploient leurs moyens de stimulation du système de soins.

\section{Méthodologie}

Si la nature de l'investigation conduite rapproche majoritairement notre méthode de l'analyse de données qualitatives, nous nous inscrivons cependant dans une approche graduée, les données sont d'abord qualitatives en ce que la qualification d'un phénomène précède toujours 
sa mesure (Campbell, 1974) et peuvent alors être exprimés en nombre (Miles et Huberman, 2003).

\subsection{Considérations générales}

Nous recueillons d'abord des récits narratifs. La mise en scène par les acteurs de leurs actions par l'intermédiaire du récit, artefact présentant pour eux l'apparence de l'objectivité, révèle à la fois la réalité perçue du quotidien des acteurs, la valeur symbolique de certaines choses et de la façon dont les acteurs assument l'incertitude dans la prise de décision (Lorino, 2005). Lorsque les acteurs expriment par exemple le fait que les chimiothérapies embolisent leur hôpital de jour, peu importe que cela soit une réalité ou simplement une perception exacerbée d'un problème mineur. C'est ainsi qu'ils le vivent au quotidien, et cela révèle les priorités symboliques qu'ils accordent à certains soins plutôt qu'à d'autres. Les données acquièrent leur force en se concentrant sur les événements qui surviennent dans le contexte quotidien des personnes concernées. L'ancrage de proximité, l'identification de phénomènes précis et contextualisés leur donne une force et contribue à décrypter la complexité qui pèse sur l'organisation réelle des soins.

Les récits narratif, alors dénommés opinions, vont être soumis à un travail de documentation, d'objectivation, ou de relativisation que ce soit par la mesure ou par la confrontation aux opinions d'autres experts dans une perspective de recherche de preuves scientifiques. Ce travail de confrontation systématique a pour objectif de pouvoir hiérarchiser ce qui relève des faits documentés, des opinions ou des sentiments des uns et des autres.

Cette confrontation entre les opinions exprimées par les acteurs de terrain et la quantification permet au chercheur d'opérer une triangulation, d'approfondir, d'initier de nouvelles façons de penser ou développer l'analyse. La construction d'une dialectique avec les acteurs de terrain permet d'affiner les chaînes de causalité pertinentes qui expliquent l'organisation actuelle et permettront de transformer de façon pertinente l'organisation future. Si la mesure de l'activité réelle révélait que peu de transfusions ont été réalisées dans l'hôpital de jour alors qu'il ressort que les acteurs ont un sentiment d'embolisation, alors la cause ne serait pas dans le nombre des transfusions à réaliser mais liés à d'autres paramètres qu'il conviendrait d'identifier.

\subsection{Nature des données exploitées et modalités de recueil des données}

La phase de recueil de données a consisté en une collection d'observations, d'entretiens et de 
recherche de documents. Observer les acteurs en situation professionnelle, organiser des entretiens avec les acteurs pertinents et examiner les documents de routine, rapports internes aux organisations concernées ou publications scientifiques venant étayer, relativiser ou invalider les opinions exprimées par les acteurs (Wolcott 1992).

Il est important par ailleurs de ne pas s'enfermer dans l'utilisation exclusive d'une source d'alimentation des données recueillies. La question déterminante est de savoir si la donnée recueillie a été obtenue de façon « ouverte », c'est à dire au su de sujets-source (obstrusive) ou « dissimulée », c'est à dire à l'insu des sujets-source (unobstrusive). (Baumard, Ibert, 2003 ) et de recouper les deux sources d'alimentation.

L'usage d'au moins deux sources pour collecter des données permet de s'affranchir, chaque fois que cela est possible, des données trop exclusivement conçues pour servir un intérêt particulier.

L'organisation des soins est un espace d'étude complexe, regroupant une multitude de problématiques éclatées entre différentes méthodologies et différentes contraintes. S'il n'est pas possible d'embrasser en une seule opération toutes les dimensions ayant un impact sur l'organisation des soins, l'analyse approfondie de cette dernière ne peut se concevoir sans l'utilisation des «briques» élaborées par les différentes disciplines dont les champs s'entrecroisent.

\subsection{Le recueil et la synthèse des opinions des acteurs}

La grille de screening que nous utilisons d'ordinaire pour conduire les entretiens semidirectifs (tableau 1) a été utilisée pour procéder à la relecture des résultats des études précedentes et des données supplémentaires. Une enquête réalisée spécifiquement auprès des infirmières libérales a complété l'étude sur certaines questions spécifiques. 
Tableau 1: Grille de screening des opinions des professionnels de santé sur l'organisation des soins

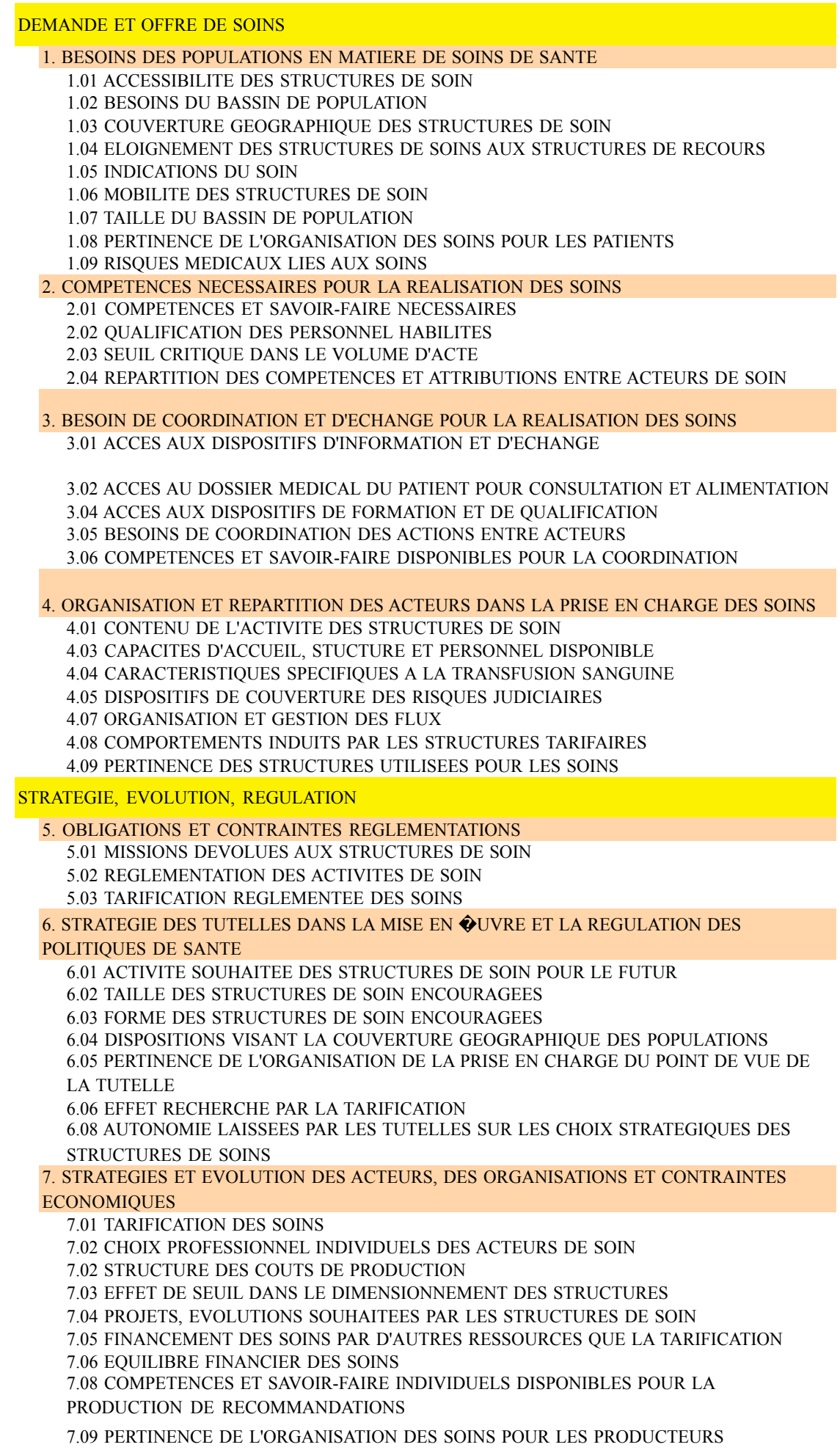

Les opinions des acteurs sont en principe recueillies en organisant des entretiens 
spécifiquement centrés sur la problématique étudiée. Dans cette étude, nous avons procédé un peu différemment. Ayant déjà recueilli à plusieurs reprises les opinions des acteurs concernés par l'organisation des soins à domicile en cancérologie (Buthion 2004, 2007, 2009), nous possédions déjà des recueils en nombre suffisant pour pouvoir dresser un inventaire des principales opinions concernant la chimiothérapie à domicile. Ce matériau permet également un repérage des modifications des opinions dans le temps. Il a été complété par des entretiens réalisés dans le cadre de l'étude OSAD auprès de 12 HAD de la région Rhônes-Alpes. Nous avons donc exploité à nouveau ce matériau pour en extraire une synthèse spécifique à ce soin.Les opinions exprimées ont ensuite été regroupées par proximité de contenu, jusqu'à permettre la rédaction par les chercheurs d'une opinion de synthèse résumant les propos tenus par les personnes interrogées. L'encadré suivant montre la forme du document permettant de synthétiser les opinions des acteurs :

\subsection{De la synthèse d'opinion à l'approche scientifique : identification des problématiques clés et recherche de preuves}

Les données acquièrent leur force en se concentrant sur les événements qui surviennent dans le contexte quotidien des personnes concernées. L'ancrage de proximité, l'identification de phénomènes précis et contextualisés leur donne une force et contribue à décrypter la complexité qui pèse sur l'organisation réelle des soins. Au delà, cela suppose que les opinions exprimées, les faits évoqués, doivent chaque fois que cela est possible être l'objet d'un travail de documentation, d'objectivation, ou de relativisation que ce soit par la mesure ou par la confrontation aux opinions d'autres experts (Figure 4). Ce travail de confrontation systématique a pour objectif de pouvoir hiérarchiser ce qui relève des faits documentés, des opinions ou des sentiments des uns et des autres, et de révéler ce qui ne semble pas spontanément préoccuper les acteurs de soins.

Figure 6: Logique de traitement des données 
Synthèse des opinions exprimées

(1) Quels sont les problématiques qui préoccupent les acteurs de soins ?

(2) Quelles sont les problématiques qui ne préoccupent pas les acteurs de soins?

\section{Documentation des opinions exprimées}

(1) Le problème exposé a-t-il été documenté de façon pertinente dans la littérature scientifique d'une ou plusieurs disciplines ou doit-il l'être de façon approfondie?

(2) La littérature révèle-t-elle des éléments non soulevés par les acteurs ? Que peuton apprendre des différences?

(3) Collecte et analyse de données complémentaires

Impact des opinions exprimées sur l'organisation des soins

(1) Le problème exposé a-t-il un impact sur l'organisation des soins et comment peut-on le modéliser?

(2) Existe-t-il des problèmes d'organisation qui ne préoccupe pas les acteurs ?

La conséquence de cette analyse est de rendre pertinente une décomposition/recomposition des assemblages préexistants, par une série.

\section{5. Étude de coûts : données économiques et financières}

Le cadre théorique de départ que nous avons utilisé pour appréhender l'analyse économique et financière est celui de la comptabilité de gestion (Bouquin, 2008). Il rend compte des consommations de ressources financières des processus de production, et celui qui en découle du contrôle de gestion, qui cherche à rapprocher ces éléments de comptabilité financière de la stratégie des organisations pour permettre de prendre des décisions qui ne soient pas strictement comptables (minimisation des coûts). La perspective adoptée est celle du financeur des dépenses de soins (nous n'avons pas retenu la perspective sociale, ni la perspective macro-économique).

Ce cadre conceptuel peut être utilisé comme un révélateur du mode de fonctionnement et des problématiques bloquant ou faisant évoluer les organisations. Lorsque l'on entre dans le monde de la santé par l'approche économique dominante, ou par la pratique des acteurs de soins, on se rend compte que le modèle qui fonctionne est souvent un modèle marginaliste. Dans une logique budgétaire de comptabilité publique, où l'investissement constitue une dépense comme une autre, l'équipement est financé en une fois. L'amortissement est une abstraction comptable et le coût qui est perçu par celui qui l'utilise est un coût marginal 
d'utilisation, celui qui va peser sur le budget du service à court terme. Il est révélateur des horizons décisionnels qui ont prévalu pendant longtemps pour les acteurs de terrain, et du peu de compte que ces derniers devaient alors rendre sur l'utilisation des équipements. Le passage à la T2A étant récent, on ne sait pas encore comment la prise en compte de l'amortissement dans la tarification conduira à modifier ce mode de gestion.

La mesure des coûts dans une approche classique de micro-costing classique, consiste souvent à considérer qu'il existerait un «coût» positif, fusse-t-il moyen, qu'il conviendrait de découvrir. Nous utilisons pour notre part le calcul de coût comme moyen de révéler le fonctionnement des organisations et la structuration de l'action collective. Le cadre d'analyse en coûts complets et en coûts variables peut devenir un révélateur du surdimensionnement des capacités mises en œuvre. L'analyse en coûts d'activité révèle les phénomènes de subventionnement, les actes gratuits, les coopérations des acteurs ou la véritable cause des surcoûts liés au rattachement de certaines structures de soin à telle ou telle institution ou tutelle (à l'hôpital, on se comporte comme si chaque séjour devait " payer » la structure de réanimation, même s'il n'en a pas l'usage). Le « target costing » devient un moyen de montrer comment la conception de certains plateaux techniques va peser sur le coût des soins pour plusieurs années, par l'effet clapet que va provoquer l'investissement dans une certaine forme de structure de soins (un hôpital de jour flambant neuf doit ensuite être rempli, on garde donc les patients plutôt que de les envoyer dans les réseaux de soins). L'analyse des marges financières révèle les logiques de subventionnement et les priorités stratégiques des acteurs. L'analyse des coûts liés aux dysfonctionnements révèle la complexité des processus dans ce secteur et l'analyse des écarts montre l'importance de l'incertitude dans le processus de transformation.

Nous avons donc privilégié une analyse de type fonction à assurer et coût complet de l'activité correspondante, pour tenter d'établir dans quelles conditions d'allocation de ressources une organisation peut prendre en charge des transfusions ambulatoires, avec un niveau de qualité défini (le même pour tous) et de façon stable dans le temps (limitant les coûts non financés, mais prévisibles). La perspective retenue, celle du financeur des soins, engage à tenir compte des dépenses qui, quel que soit le moment où elles devront être engagées, seront prises en charge par le financeur. Ainsi, lorsqu'un soin peut nécessiter le recours à des structures d'urgence comme le Samu, il est important dans la structure de coût du soin, que le recours possible à ce service soit considéré comme un élément de coût, même si les financements sont séparés et qu'aucun échange financier n'a lieu entre le SAMU et la 
structure de soins. Le recours au Samu ne rentrera alors pas dans le tarif payé à la structure dispensant le soin, mais elle fait partie de l'analyse économique du financeur lorsqu'on souhaite éclairer les différents modes d'organisation des coûts qu'elles génèrent pour le financeur.

Nous avons, pour construire ce raisonnement, utilisé les informations fournies par les différents acteurs de soin et comparer les approches des différentes structures. Nous aboutissons à des résultats que nous nommons "estimation», afin de bien les distinguer d'une donnée positive qui révélerait une réalité immuable.

\subsection{Discussion et recommandations}

Nous discuterons de chacun des résultats de l'étude et des perspectives qu'elle présente, ainsi que de notre propre point de vue de chercheur, forgé au fil de l'analyse.

Il est de coutume de présenter la discussion séparément de l'exposé des données de l'études dans le classique plan [Matériel et Méthode-Résultat-Discussion]. Le respect de cette progression chronologique a pour objectif de laisser au lecteur le temps de se faire une opinion avant de lire les conclusions des auteurs de l'étude, qui doivent, par honnêteté intellectuelle et scientifique, exposer les limites des investigations qu'ils ont menées.

Cette chronologie sera partiellement bouleversée. La recherche en sciences de gestion, telle que nous la concevons, a une visée "prescriptive», au sens où elle cherche à fonder une modification de l'action collective. Il est donc d'autant plus fondamental, dans la mesure où cette posture conduit à émettre des opinions sur les zones d'ombre ou l'analyse scientifique ne permet pas d'apporter de preuves incontestées (à défaut de n'être jamais véritablement incontestables). L'explicitation de toutes les hypothèses formulées doit permettre au lecteur de faire la part des choses entre la collecte et l'analyse des données, et l'usage que le chercheur en fait pour formuler des conclusions. La discussion est donc intégrée à chaque résultat présenté. L'ensemble du matériau utilisé est présenté et détaillé dans l'annexe. 
Page 25 sur 122 


\section{Deuxième Partie : Résultats}

Nous présentons dans cette partie l'ensemble des résultats de l'étude. Le lecteur trouvera les annexes référencées dans lesquelles tout le détail des données et de leur traitement figure.

\section{Synthèse des opinions : croyances, contradictions, interrogations}

Nous présentons ici les principales idées qui se dégagent de la synthèse des opinions des acteurs, et ont constitué les hypothèses soumises à documentation. L'intégralité de la synthèse figure en annexe 1 .

$\underline{\text { Les acteurs des soins à domicile souhaiteraient pratiquer plus de chimiothérapies à domicile, }}$ mais les demandes stagnent, voire régressent au fil des années. De l'avis des acteurs des soins à domicile, cette activité est très difficile à développer, alors qu'elle a beaucoup fait parler en cancérologie. Nous avons été amenés à nous interroger sur l'ampleur du phénomène, alors qu'au début de nos travaux sur les soins à domicile, cette tendance à l'externalisation était considérée comme une tendance de fond. Par ailleurs, lorsque le patient se rend à l'hôpital, il est possible de regrouper visites et soins dans la même journée, et cela permet de mutualiser des frais de transport élevés, mais incontournables pour certaines prestations. Nous avons donc réalisé un dénombrement aussi exhaustif que possible des chimiothérapies réalisées en ambulatoire dans les structures hospitalières, qui ont été réalisées sans autres actes de soins que l'administration de la molécule de chimiothérapie et donc potentiellement externalisables à domicile (point 2).

$\underline{\text { La résistance des médecins prescripteurs, la réglementation et le passage à la tarification à }}$ $\underline{\text { l'activité sont les deux raisons invoquées pour expliquer l'absence de véritable demande de la }}$ part des hôpitaux. Dans les arguments invoqués par les médecins figurent en bonne place la crainte de ne pas avoir une qualité de soins équivalente. Nous avons investigué la littérature sur la chimiothérapie à domicile pour vérifier si ces craintes avaient un quelconque fondement dans la littérature médicale. Nous nous sommes également interrogés sur les risques potentiels de perte de compétences que pouvait induire la faiblesse de l'activité à domicile (point 3).

Une chimiothérapie peut être d'une durée très variable, allant d'une simple injection à une perfusion de plusieurs heures. Les infirmières libérales ne semblent pas toujours prêtes à $\underline{\text { rester mobiliser de longues heures au chevet du patient à domicile, les chimiothérapies qui }}$ 
leur sont proposées ne dépassent en général pas 1h30. Dans certaines zones géographique cependant, mobiliser une infirmière libérale pendant plus d'une heure pour un seul patient relève de l'exploit tant ces dernières sont saturées par l'activité de soins. Du côté des infirmières libérales, la situation est également différente selon les zones géographiques. Du côté des infirmières libérales, certaines déplorent de ne pas être plus sollicitées pour prendre en charge des chimiothérapies complètes à domicile et trouvent peu valorisant de se voir reléguées à venir débrancher des perfusions posées à l'hôpital. Pourtant, beaucoup de libéraux (médecins, infirmières) apprécient d'être impliqués dans la prise en charge du cancer de patients dont ils se sont occupés dans le passé, et dont ils devront assurer les suites de la prise en charge, après le cancer ou pendant la phase de dégradation et de fin de vie. Sur certaines zones géographiques, certaines n'ont jamais cherché à se faire habiliter pour la chimiothérapie, faute de n'avoir jamais été sollicitées. Nous nous sommes donc interrogés sur le frein que pouvait constituer le refus des infirmières de monter en charge sur ces activités. Un questionnaire administré auprès d'infirmières libérales nous a permis de vérifier la disponibilité des infirmières libérales du département du Rhône, où la chimiothérapie à domicile est pratiquée depuis plusieurs années (point 4).

Il existe des croyances très ancrées sur le coût de la chimiothérapie à domicile, soit pour dire qu'elle coûte moins cher qu'à l'hôpital, soit pour affirmer le contraire. Les deux composantes évoquées pour faire pencher la balance d'un côté ou de l'autre sont les coûts des transports d'une part, et le temps passé et non mutualisé par les infirmières libérales qui interviennent au domicile des patients, comparés aux hôpitaux de jour. Aucune étude sérieuse et actualisée ne permet à ce jour d'affirmer que l'une ou l'autre de ces hypothèses soit valides. Nous avons donc mené une investigation approfondie des données de prix et de coûts des différentes alternatives (point 5).

Selon les acteurs des réseaux et des $\mathrm{HAD}$, les patients sont demandeurs de soins à domicile, surtout quand ils demeurent très loin de l'hôpital. Selon les médecins des hôpitaux, les patients préfèrent le côté rassurant de l'hôpital. Une étude reposant sur les méthodes de révélation des préférences des patients et la méthodologie de la disposition à payer est en cours sur les soins à domicile dans le même périmètre que celui que nous avons étudié. Ces résultats devraient être disponibles fin 2010. La tendance sur les 115 premiers patients ayant répondu au questionnaire ne montre pas de tendance générale très tranchée. Les souhaits des patients sont très variables selon le patient, son entourage, le type de soins à recevoir et l'expérience qu'ils ont déjà eu de soins à domicile. 
Enfin, la chimiothérapie évolue et les laboratoires pharmaceutiques mettent de plus en plus souvent sur le marché des formulations « orales », qui peuvent être administrées par le patient lui-même, sans recours à un personnel soignant. Les impacts de cette formulation sur l'organisation des chimiothérapies et l'effet potentiel sur l'activité des hôpitaux de jour sont actuellement inconnus et impossible à évaluer.

\section{La faiblesse de la pratique de la chimiothérapie à domicile}

Il existe un GHM unique, le [28Z07Z chimiothérapie pour tumeur, en séances], qui permet de rémunérer la séance, classé dans la CMD des séjours moins 24h). Avant 2005, le motif chimiothérapie était inclus dans le GHM 24Z02Z dans les CMD* «séances et séjours de moins 2 jours ». De 2006 à 2009, ces séjours sont passés de 1677711 à 1925 656, soit une augmentation de près de $15 \%$. L'intégralité des données de base et de leur analyse sont présentées dans l'annexe 2 .

Le tableau 2 donne une idée de la répartition 2009 par type d'hôpitaux de la base publique et PSPH. Ces données confirment que se sont essentiellement les structures techniques les plus grosses qui prennent en charge pour l'essentiel cette prestation. Les gros $\mathrm{CH}$, les $\mathrm{CHR} / \mathrm{U}$ et les CLCC en assurent $85 \%$.

Tableau 2 : Répartition des séjours en 2009 de la base public.

\begin{tabular}{|l|r|r|}
\hline \multicolumn{1}{|c|}{ Opérateurs } & \multicolumn{2}{|c|}{2009} \\
\hline CH supérieur à 16 000 séjours & 426783 & $35,17 \%$ \\
\hline CHR/U & 368684 & $30,38 \%$ \\
\hline CLCC & 251684 & $20,74 \%$ \\
\hline PSPH & 92579 & $7,63 \%$ \\
\hline CH de 10 001 à 16 000 séjours & 46610 & $3,84 \%$ \\
\hline CH de 5501 à 10 000 séjours & 21940 & $1,81 \%$ \\
\hline CHR de moins de 5500 séjours & 5214 & $0,43 \%$ \\
\hline TOTAL & 1213494 & $100,00 \%$ \\
\hline
\end{tabular}

Nous observons également que les CLCC sont placés en troisième position en ce qui concerne le nombre de séances, pour des raisons de nombre et de taille de ces structures. On dénombre actuellement seulement 20 CLCC contre plus de $450 \mathrm{CH}$ (Fédération Hospitalière Française).

A l'hôpital, le séjour sera classé dans ce GHM pour toutes les chimiothérapies réalisées en hôpital de jour, quelle que soit leur durée, et compte tenu de l'existence d'autres soins et

\footnotetext{
${ }^{*}$ CMD : Catégorie Majeur de Diagnostic
} 
examens qui peuvent être réalisés concomitamment. Se rendre à l'hôpital peut permettre au patient de bénéficer des soins dans la même journée. Nous avons procédé à une analyse systématique des actes classants et non classants des bases ATIH, en classant les actes relevant de la chimiothérapie elle-mêmes (injections de produits anticancéreux) et les autres.

Nous avons comptabilisé tous les actes qui n'étaient pas des chimiothérapies, et gommé l'effet de dénombrement des actes qui constituent des compléments à d'autre, comme par exemple une anesthésie concomitante à une intervention chirurgicale, une reconstitution tridimensionnelle d'image en radiologie. Compte tenu de la modification du GHM intervenue à partir de 2006, nous avons mené l'analyse sur les années 2006-2009. Nous ne connaissons pas la répartition moyenne des actes réalisés en plus de la chimiothérapie, et avons donc arbitrairement considéré qu'ils étaient répartis sur différents séjours. Cela nous permet de déterminer a minima le nombre de séance où le patient n'est venu que pour recevoir sa ou ses molécules de chimiothérapie.

Par ailleurs, la visite au cancérologue, lorsqu'elle est réalisée lors de la journée d'hospitalisation de jour pour chimiothérapie, ne fait pas l'objet d'une facturation supplémentaire. La tracer dans les données de l'ATIH n'est donc pas possible. Cependant, l'observation de certaines situation nous donnent à penser que cette visite serait reportée par rapport à la réalisation de la chimiothérapies afin de pouvoir être facturée en sus, mais nous n'avons pas pu mesurer l'ampleur réelle de cette pratique.

Tableau 3 : dénombrement des séances réalisées pour seul motif de chimiothérapie

\begin{tabular}{|c|c|c|c|c|c|c|c|c|}
\hline & \multicolumn{4}{|c|}{ Public et PSPH } & \multicolumn{4}{|c|}{ Privé } \\
\hline & $\begin{array}{c}\text { Nombre de } \\
\text { séjour dans la } \\
\text { base ATIH }\end{array}$ & \begin{tabular}{|c|} 
Actes autres \\
que \\
chimiothérapie \\
réalisés dans \\
les séjours
\end{tabular} & \begin{tabular}{|c|} 
Nombre \\
d'actes \\
rapportés au \\
nombre de \\
séjours
\end{tabular} & $\begin{array}{l}\text { Nombre de } \\
\text { séjour pour } \\
\text { seule chimio }\end{array}$ & $\begin{array}{c}\text { Nombre de } \\
\text { séjour dans la } \\
\text { base ATIH }\end{array}$ & \begin{tabular}{|c|} 
Actes autres \\
que \\
chimiothérapie \\
réalisés dans \\
les séjours
\end{tabular} & $\begin{array}{l}\text { Nombre } \\
\text { d'actes } \\
\text { rapportés au } \\
\text { nombre de } \\
\text { séjours }\end{array}$ & $\begin{array}{l}\text { Nombre de } \\
\text { séjour pour } \\
\text { seule chimio }\end{array}$ \\
\hline 2006 & 972026 & 191890 & $19,74 \%$ & 780136 & 705685 & \begin{tabular}{r|}
18903 \\
\end{tabular} & $2,68 \%$ & 686782 \\
\hline 2007 & 1017031 & 186212 & $18,31 \%$ & 830819 & 707236 & 20480 & $2,90 \%$ & 686756 \\
\hline 2008 & 1120087 & 174056 & $15,54 \%$ & 946031 & 719685 & 19618 & $2,73 \%$ & 700067 \\
\hline 2009 & 1213658 & 167518 & $13,80 \%$ & 1046140 & 711998 & 19176 & $2,69 \%$ & 692822 \\
\hline
\end{tabular}

On constate une présence beaucoup plus forte d'autres actes dans les séjours du secteur public et PSPH que dans le secteur privé. Cependant, si le ratio du nombre d'autres actes sur nombre de séjour est resté stable dans le secteur privé, il décroît fortement dans le secteur public. On y voit sans doute les traces d'une préoccupation quant à la gestion des séjours facturables. Rémunérées forfaitairement quels que soient les autres actes réalisés, les structures hospitalières n'ont pas intérêt de regrouper des prestations de soins qui ne modifient pas la 
rémunération du séjour. Le regroupement est donc réservé aux patients qui ont de longs déplacements depuis leur domicile.

Cette estimation permet de déterminer que la grande majorité des séjours (en 2009 86,2\% dans le secteur public et PSPH et 97,31\% dans le secteur privé) ne sont réalisés que pour administrer les molécules.

Du côté des soins à domicile, deux acteurs sont identifiés : les HAD et les réseaux de soins. Bien qu'ils puissent apparaître comme concurrent dans la prise en charge, leur rôle est très différents. Les HAD ont été crée pour représenter une alternative aux hospitalisations longues, et pas aux hospitalisations de jour. La chimiothérapie à domicile ne pouvant être effectuée que dans le cadre d'un réseau de soins ou d'une HAD, ces dernières sont intervenues assez naturellement, et se trouve parfois en position de prendre en charge, à la place de l'hôpital de jour, des séances en ambulatoire. Cette tâche devrait logiquement être dévolue aux réseaux, mais ces derniers sont peu nombreux sur le territoire. Cependant, la longueur des hospitalisations à domicile pour seul motif de chimiothérapie (3,8 jours en 2009) peut également laisser penser que ce ne sont pas les mêmes profils de patients que ceux qui sont pris en charge dans les réseaux ou dans les hôpitaux de jour.

En HAD la chimiothérapie peut être réalisée à titre de prise en charge principale, associée ou non à d'autres soins, ou à titre de prise en charge associée. $\mathrm{K}$ indique l'indice de Karnosky, qui mesure l'état de dépendance de la personne. Un indice de 70 indique une personne qui peut assurer ses soins, mais incapable d'exécuter une activité normale ou de faire un travail actif. Un indice de 50 indique une personne dans une dépendance totale, exigeant une assistance considérable et un soin médical fréquent. On trouvera dans cette catégorie des patients dans un état de santé dégradé, qui sont entrés dans une logique de prise en charge à visée plus palliative, où la chimiothérapie est associée à d'autres soins.

Les patients susceptibles de recevoir leurs chimiothérapies en hôpital de jour, et de ne s'y rendre que pour cela, sont les patients inclus en HAD pour seul motif de chimiothérapie. Malgré un doublement du nombre de séjours (de 6497 à 12422) sur les cinq années étudiées, la chimiothérapie à domicile pratiquée par les HAD est très faible par rapport au 1,6 millions identifiées comme potentiellement externalisables dans le paragraphe précédent.

Le second intervenant potentiel pour la chimiothérapie à domicile est le réseau territorial de cancérologie. Il n'existe pas de bases statistiques centralisées, aussi est-il difficile de connaître les volumes de chimiothérapie qui y sont réalisées. En 2005, un rapport de la Haute Autorité 
de Santé faisait état de 4 réseaux pratiquant la chimiothérapie à domicile. Nous avons cherché les informations sur internet, "réseaux territoriaux de cancérologie ou oncologie», puis contacté ceux qui faisait état d'expérience de chimiothérapie à domicile. En sus du réseau Source à Lyon, nous en avons contacté 5. Les informations que nous avons pu collecter concernant les chimiothérapies complètes (administration des molécules intégralement à domicile) sont rassemblées dans le tableau 4.

Tableau 4 : Chimiothérapies à domicile identifiée dans les réseaux

\begin{tabular}{|l|r|r|}
\cline { 2 - 3 } \multicolumn{1}{c|}{} & $\mathbf{2 0 0 8}$ & $\mathbf{2 0 0 9}$ \\
\hline Source (Lyon) & 35 & 532 \\
\hline OncoLoire (Saint & & \\
Nazaire, St Herlin)) & 357 & 464 \\
\hline Onco Loire Estuaire & & 1229 \\
\hline TOTAL & $\mathbf{3 9 2}$ & $\mathbf{2 2 2 5}$ \\
\hline
\end{tabular}

La pratique de la chimiothérapie via les réseaux de soins est très faiblement développée. Lorsqu'elle se développe, elle reste assez artisanale en volume.

Nous récapitulons dans le tableau 5 le dénombrement des séances de chimiothérapie « simples », c'est-à-dire non accompagnées d'autres soins réalisés de façon concomittante.

Tableau 5 : récapitulatif des séances de chimiothérapie ambulatoire « simples » réalisées par les différents opérateurs

\begin{tabular}{|l|r|r|r|r|r|r|r|r|}
\hline & \multicolumn{2}{|c|}{2006} & \multicolumn{2}{c|}{2007} & \multicolumn{2}{c|}{2008} & \multicolumn{2}{c|}{2009} \\
\hline $\begin{array}{l}\text { Hôpitaux } \\
\text { Publics et } \\
\text { PSPH }\end{array}$ & 780136 & $52,8 \%$ & 830819 & $54,4 \%$ & 946031 & $57,1 \%$ & 1046140 & $59,7 \%$ \\
\hline $\begin{array}{l}\text { Hôpitaux } \\
\text { privés }\end{array}$ & 686782 & $46,5 \%$ & 686756 & $45,0 \%$ & 700067 & $42,3 \%$ & 692822 & $39,5 \%$ \\
\hline HAD & 9330 & $0,6 \%$ & 8400 & $0,6 \%$ & 8993 & $0,5 \%$ & 12423 & $0,7 \%$ \\
\hline $\begin{array}{l}\text { Réseaux de } \\
\text { Cancérologie }\end{array}$ & & & & & 392 & $0,0 \%$ & 2225 & $0,1 \%$ \\
\hline TOTAL & 1476248 & $100 \%$ & 1525975 & $100 \%$ & 1655483 & $100 \%$ & 1753610 & $100 \%$ \\
\hline
\end{tabular}

La chimiothérapie à domicile représentait en 2009 moins de 1\% des prises en charge. Les réseaux ont pour le moment une place insignifiante. Leur émergence tardive dans le paysage sanitaire peut expliquer leur faible place, mais ce qui se passe dans les lieux où ils sont présents tendrait à montrer que leur existence ne suffit à provoquer une externalisation importante. Ce sont les hôpitaux publics et PSPH qui montent fortement en charge sur cette 
prestation.

\section{La qualité de la chimiothérapie et l'expérience des infirmières}

La littérature confirme que le traitement à domicile peut être administré de façon sûre et n'entraînent pas d'accidents graves, à condition qu'il y ait un critère de sélection des patients et qu'une bonne organisation des soins soit garantie. La plupart des auteurs évoquent une meilleure qualité de vie pour le patient dans les soins de chimiothérapie à domicile. Les études sur la qualité de vie sont cependant faiblement documentées. L'intégralité de l'étude de littérature figure en annexe 3.

La chimiothérapie à domicile fait intervenir plusieurs acteurs. Son organisation complexe et demandant une rigueur irréprochable pour garantir le maximum de sécurité au patient, nécessite une qualification particulière. Face à la diversité et à la durée des traitements anticancéreux, ainsi qu'aux différentes compétences des infirmiers, nous avons voulu explorer la pertinence de la notion de seuil critique en terme de pratique de chimiothérapie par infirmière en fonction du temps. Au delà du processus de qualification, faut-il s'assurer que l'infirmier(ère) pratique de façon régulière ou, peut-on laissé l'IDE (Infirmier Diplômé d'Etat) exercer seulement avec une qualification validée sans mise à jour des connaissances et/ou compétences ? De manière plus générale, ce travail a pour but de faire un état des lieux de l'effet du volume des pratiques sur la sécurité des chimiothérapies, compte tenu de la faiblesse des volumes de chimiothérapie à domicile. L'intégralité de notre investigation figure en annexe 4.

Le Réseau Territorial de Cancérologie Source à Lyon a réalisé 532 séances de chimiothérapie à domicile pendant l'année 2009, réparties sur 69 patients, mobilisant donc au maximum 69 infirmières, soit une moyenne de 7,7 injections par infirmière. Le Centre Léon Bérard à Lyon a réalisé 17866 séjours pour chimiothérapies en séance (Source ATIH) dans son hôpital de jour pour un effectif moyen de 24 infirmières soit environ 744 séances par infirmière et par an. Dès lors, le nombre moyen d'actes effectués par les infirmiers (ères) libéraux (ales) est-il suffisant pour ne pas être déqualifié?

Dans notre enquête réalisée auprès des infirmières -, une majorité $(75 \%)$ des infirmières accréditées n'a jamais vécu d'incidents pendant leur pratique. Une seule infirmière (sur 37) a vécu une complication grave (choc anaphylactique). Les autres accidents sont minimes et sans complications majeures pour le patient. Ces résultats montrent que la pratique actuelle n'a pas de raison d'être source d'inquiétude de la part des infirmières libérales. Dans l'étude réalisée 
auprès des infirmières, la moyenne du nombre d'années d'exercice sans incidents est a peu près de 7 années $(n=28)$. Nous constatons alors que la proportion d'infirmières $(n=9)$ qui ont vécu un incident $(24,3 \%)$ pendant leur pratique n'est pas très significative; il aurait été intéressant d'avoir un plus grand échantillon. Cependant, au regard du nombre important d'effets secondaires des produits cytotoxiques, avoir eu un incident non grave (sans complications majeurs pour le patient) en dix années de pratique par exemple ne semble pas alarmant. (cf annexe 5)

Dans la littérature, la notion de « seuil critique » (c'est à dire le nombre d'acte en dessous duquel le professionnel perd en qualification) dans la pratique de la chimiothérapie à domicile n'est pas évoquée. Nos investigations sur les notions de seuil critique nous amènent à conclure que la pratique régulière de la chimiothérapie à domicile pourrait être moins nécessaire qu'en milieu hospitalier du fait de la simplification des exigences .

Tous ne sont pas admissibles en fonction de leurs antécédents, de la molécule et de leur état clinique. La première cure de chimiothérapie est faite à l'hôpital et pour les autres cures qui seront faites à domicile, la molécule reste la même. Cela permet de déceler d'éventuels risques et de vérifier la tolérance au traitement. La préparation sous contrôle des molécules anticancéreuses est faite par une pharmacie hospitalière. L'infirmier ne manipule aucune préparation des traitements cytotoxique, ce qui réduit considérablement le risque d'accident. Enfin, son rattachement obligatoire à une HAD ou un réseau de soins pour administrer des chimiothérapies le met de fait en contact permanent avec des infirmières de coordination.

La pratique faible et irrégulière dans l'administration de traitement cytotoxique chez les infirmiers libéraux par rapport aux infirmiers en milieu hospitalier, ne semble pas être un frein pour continuer d'exercer, ni un problème majeur pour la qualité des soins. L'IDE qui n'a pas suivi de mise à jour de ses connaissances ou de formation continue depuis son habilitation (formation spécifique ou diplôme post 1992), n'est pas interdite de pratiquer la chimiothérapie à domicile, les informations nécessaires pouvant être amenées par la coordination des soins de la structure de rattachement qui la sollicite.

Il n'y a donc pas véritablement de fondements aux inquiétudes des médecins

\section{L'activité de chimiothérapie à domicile : des infirmières disponibles}

Nous avons interrogé une cinquantaine d'infirmières dans le département du Rhône. Nous constatons à l'unanimité des infirmières réalisant la chimiothérapie à domicile, le souhait 
d'augmenter cette activité. Il ressort d'une manière générale une réelle envie de s'investir et de pratiquer plus, surtout d'avoir une prise en charge globale. Cette pratique pourrait se développer autant en milieu rural qu'en milieu urbain. Le nombre de chimiothérapie ne dépend pas du milieu d'exercice. Les résultats semblent indiquer une quantité à peu près similaire $(43,5 \%$ contre $56,5 \%)$ concernant les actes de chimiothérapie réalisés respectivement en milieu urbain et en milieu rural. Ainsi, le fait de travailler en milieu rural ne favorise pas l'augmentation du volume d'actes. La densité de population en milieu rurale peut être à l'origine de ce phénomène, mais cela montre également que les soins à domicile sont tout autant proposés aux patients qui habitent à proximité des Etablissements de Soins qu'à ceux qui en sont éloignés, ce qui relativise quelque peu les discours sur les économies potentielles de frais de transport.

Environ $70 \%$ considèrent que le nombre de séances de chimiothérapie a diminué ou stagné ces dernières années. Certaines infirmières interrogées dénoncent une logique économique. Elles indiquent que l'instauration de la T2A en 2005 a changé le fonctionnement des établissements hospitaliers en termes d'activité. Elles évoquent le fait que les hôpitaux ou cliniques ne " jouent pas le jeu » et " gardent les patients pour eux». Ceci tend également à monter que l'encombrement des structures hospitalières ne semble pas aussi important que cela a pu être évoqué par certains acteurs, ou que ces derniers souhaitent se décharger d'autres choses que de cette prestation.

Un terme récurrent, celui du manque de coordination entre les différents acteurs de soins, est évoqué par la plupart des infirmières. Elles soulignent le manque de communication entre le milieu hospitalier et le milieu libéral. Elles ajoutent vouloir plus de relation et de disponibilité de la part des prestataires et des médecins. Les actions de coordination sont dans l'ensemble jugées moyennes voire insuffisantes. Face à un discours parfois dur sur les libéraux qui sont très peu disponibles, ceci tend à nous interroger sur un manque de compétence en coordination ou un manque d'intéressement et d'investissement de la part des différents acteurs à l'hôpital ou aux interfaces. La position de sous-traitant du travail hospitalier, à qui on confie les tâches peu intéressantes comme les suivi et les débranchements de perfusions posées à l'hôpital, n'est pas toujours appréciée.

\section{La question du coût pour l'assurance maladie : une question difficile à trancher}

Le cancérologue, qui est en général rattaché à une structure hospitalière, détermine le traitement à administrer, et lui seul interviendra pour modifier les molécules et les dosages. Le 
patient le rencontrera régulièrement pour faire le point sur l'ensemble de son traitement et le traitement des effets secondaires liés à la toxicité des molécules. Le traitement va comporter plusieurs injections appelées «cures ». Une fois l'ordonnance réalisée, la première cure aura lieu à l'hôpital, à proximité de l'oncologue prescripteur, les séances suivantes pouvant ensuite être réalisées à domicile. Le patient ne devra pas systématiquement revoir son cancérologue à chaque cure.

Quel que soit le lieu d'administration, la cure se déroulera selon le même protocole organisationnel. La veille ou l'avant-veille de la cure, le patient se rend au laboratoire d'analyse médicale pour une prise de sang, les résultats sont communiqués aux équipes médicales qui le suivent et un médecin doit, au regard de paramètres biologiques et (si besoin) de l'examen du patient, donner le feu vert pour le déroulement de la cure. Seule une molécule, l'Herceptine, ne nécessite pas de feu vert du médecin. La (ou les ) molécule(s) est (sont) alors commandée(s) à une Pharmacie Hospitalière. Le patient se rend à l'hôpital de jour, ou l'équipe de soin à domicile (HAD ou Réseau) organise la venue de l'infirmière et l'acheminement de la molécule chez le patient. Le produit est administré par l'infirmière libéral. Le dossier du patient est mis à jour après retour de l'information sur le bon déroulement de l'administration du produit par l'IDE libérale.

L'injection d'une chimiothérapie peut être courte (une injection par seringue) ou longue (certaines chimiothérapies se diffusent sur $24 \mathrm{~h}$ ou plus). Les chimiothérapies intraveineuses qui sont réalisées à domicile sont des injections ou des perfusions qui ne dépassent pas une heure et demi. Lorsque les chimiothérapies sont très longues, il arrive de plus en plus que la perfusion soit posée à l'hôpital et que le patient rentre chez lui pour être débranché lendemain ou surlendemain par une infirmière libérale.

\subsection{La séance de chimiothérapie : somme des facturations pour l'assurance maladie}

En 2006, les possibilités ont été restreintes à la réalisation dans trois cadres organisationnels bien précis : l'Etablissement de Soins public ou privé, l'Hospitalisation à Domicile ou les Réseaux de Soins. La molécule est préparée de façon centralisée par une Pharmacie Hospitalière, puis « revendue » pour être administrée à domicile. En 2006, la chimiothérapie réalisée en ambulatoire dans les hôpitaux de jour a été isolée dans le PMSI dans un GHM unique, le [28Z07Z Chimiothérapie en séance]. Ce dernier fourni depuis une base d'information permettant une certaine continuité dans l'analyse, le passage progressif à la T2A depuis 2005 obligeant les hôpitaux à renseigner le système d'information de façon plus 
fiable. L'Echelle Nationale des Coûts hospitaliers pour 2007, désormais disponible, permet de questionner les charges engagées pour ce séjour, et de les comparer aux autres modes d'administration.

Les différentes modalités de tarification des quatre solutions existant pour l'administration des chimiothérapies sont présentées dans le tableau 6. Les tarifs hospitaliers retenus sont les tarifs de référence nationaux pour la T2A 2010. Certains ajustements sont spécifiquement apportés comme le correcteur géographique, particulièrement en région parisienne. Cependant, notre réseau comparateur est un réseau lyonnais. Il peut donc être comparé au tarif de référence national. Le détail du calcul figurant dans le tableau est donné dans le point suivant.

Tableau $6:$ structure de tarification des chimiothérapies

\begin{tabular}{|c|c|c|c|c|c|}
\hline $\begin{array}{l}\text { Mode d'organisation de } \\
\text { hp rise en charge }\end{array}$ & $\begin{array}{c}\text { Structure tarifaire vish le pour l'assurance } \\
\text { mahdie }\end{array}$ & \multicolumn{2}{|c|}{$\begin{array}{l}\text { Total de la } \\
\text { prestation }\end{array}$} & \multicolumn{2}{|c|}{$\begin{array}{c}\text { Total de la } \\
\text { prestation avec } \\
\text { analyse de sang si } \\
\text { non inc huse dans le } \\
\text { totalp récédent }\end{array}$} \\
\hline $\begin{array}{l}\text { A. Structure hospitalière } \\
\text { type CHU, CH, Hôpital de } \\
\text { cancérologie }\end{array}$ & $\begin{array}{l}\text { T2A } 2010: 379,14 € \text { la séance en ambula toire } \\
\text { (hôpital de jour) }\end{array}$ & $379,14 €$ & $379,14 €$ & $405,60 €$ & $448,80 €$ \\
\hline $\begin{array}{l}\text { B. Structure hospitaliè re } \\
\text { puivée (clinique) } \\
\text { (proximité du patient de } \\
30 \mathrm{~km} \text { ) }\end{array}$ & $\begin{array}{l}\text { T2A } 2010 \text { : } 286,67 €+\text { éve ntuellement des } \\
\text { honoraires médicaux de } 23 € \text { pour une } \\
\text { consultation de spécialiste }\end{array}$ & $286,67 €$ & $309,67 €$ & $313,13 €$ & $356,33 €$ \\
\hline C. $\mathrm{HAD}$ & $\begin{array}{l}\text { Pour une prise en charge pour se ul motif de } \\
\text { chimiothérapie, théoriquement, } 181,28 € \text { pour } \\
\text { une joumée sur la base d'un patient avec un IK } \\
\text { de } 70 \text { (moyenne des IK des patients en } \\
\text { chimiothérapie à domicile). Durée moyenne } \\
\text { des séjours en HAD pour seul motif } \\
\text { d'hospitalisation de } 3,8 \text { jours. }\end{array}$ & $181,28 €$ & $688,86 €$ & $362,56 €$ & $688,86 €$ \\
\hline $\begin{array}{l}\text { D. Domicile coordonné } \\
\text { par un rése au te rnitorial de } \\
\text { cancérologie }\end{array}$ & $\begin{array}{l}\text { de } 174,38 € \text { à } 228,38 € \text { pour l' administration et } \\
\text { coordination hors moléc ule, pour un patient } \\
\text { habitant à moins de } 10 \mathrm{~km} \text { de l'infirmière }\end{array}$ & $174,38 €$ & $228,38 €$ & $200,84 €$ & $298,04 €$ \\
\hline
\end{tabular}

A l'hôpital, la tarification du séjour est forfaitaire, quels que soient leur durée, et rémunère également les autres soins et examens qui peuvent être réalisés concomitamment. De nombreuses molécules (mais pas toutes) sont facturées en sus (molécules dites sur la liste en sus). La plupart du temps, l'analyse de sang est faite en ville par le patient et facturée en plus à l'assurance maladie. Il arrive cependant dans certains cas qu'elle soit réalisée lors de l'arrivée à l'hôpital. 
Le cas de l'Hospitalisation à Domicile est assez particulier et l'analyse difficile en raison de l'absence de détails sur les actes pratiqués. Sur la base des statistiques PMSI 2009 les séjours pour seul motif de chimiothérapie ont une moyenne de 3,8 jours, ce qui représente une tarification moyenne assez élevée. La tarification inclut tout autres soins réalisés et tous les médicaments délivrés pendant le séjour. Suivant les HAD, la prise en sang est incluse (le séjour commence la veille et le service envoie quelqu'un au domicile du patient pour effectuer la prise de sang). D'autres ne l'inclus pas, (le patient va au laboratoire, l'HAD n'hospitalise qu'une journée et n'intervient qu'un jour pour la chimiothérapie). Les cures de plusieurs injections sur plusieurs journées successives vont sans doute générer des hospitalisations à domicile en continues sur toute la durée de la cure.

La chimiothérapie à domicile ne pouvant être effectuée que dans le cadre d'un réseau de soins ou d'une HAD, ces dernières interviennent par défaut en l'absence de réseau, ces derniers étant peu nombreux sur le territoire. Elles devraient normalement dans l'avenir leur laisser la place pour tous les cas ou la chimiothérapie ne nécessite pas une hospitalisation de plusieurs jours et conserver la réalisation des chimiothérapies au sein des prises en charge motivées par d'autres considérations.

La tarification est d'ailleurs parfaitement inadaptée pour ce type de soins. Actuellement, une journée d'hospitalisation en HAD pour seule motif de chimiothérapie, pour un patient parfaitement valide, pourrait être facturée en 2010 à 149,33€, soit moins que ce que coûte la plus courte des chimiothérapie en réseau. Or l'HAD est un établissement de santé, avec une structure de fonctionnement plus lourde qu'un réseau. Nous ne concevrons donc pas l'HAD comme une modalités organisationnelle à comparer au réseau de soins.

La comparaison des structures tarifaires existant ne permet pas de se prononcer en raison de l'hétérogénéité des prestations qui sont incluses dans les tarifications hospitalières. Il est possible d'approcher des éléments de comparaison plus pertinents en utilisant l'échelle nationale des coûts hospitaliers 2007 publié sur le site de l'ATIH.

\subsection{La décomposition des coûts de la chimiothérapie en réseau}

Le réseau Source, Réseau Territorial de Cancérologie, créée en 2007, a pour vocation de prendre en charge tout patient atteint de cancer du début à la fin de la maladie, en partenariat avec les autres acteurs de santé. Le réseau peut assurer la coordination de soins depuis le diagnostic, en passant par les traitements curatifs comme la chimiothérapie, les soins de support et/ou les soins palliatifs. Le projet de ce réseau a été porté par le Centre Léon Bérard, 
Centre de Lutte Contre le Cancer de Lyon et un certain nombre de professionnels libéraux (médecins, infirmiers(ères), pharmaciens, biologistes) implanté sur les territoires 7, 8, 9 et 10 du SROS III de la région Rhône-Alpes. Ce territoire représente un bassin de population de près de 2 millions d'habitants dans des zones hétérogènes : urbaines (Lyon) et rurales (Monts du Lyonnais, Nord Isère et Ouest de l'Ain...). Le Réseau Source a été agréé en décembre 2007, il a commencé son activité en juin 2008 et les premiers patients ont été pris en charge à partir de septembre 2007. Dès le mois de novembre 2007, des chimiothérapies « entièrement réalisées à domicile » ont été réalisées à titre expérimental. Devant la demande importante des patients, la décision a été prise de faire de cette activité une priorité de développement pour l'année 2009. Ainsi, après avoir réalisé 35 séances de chimiothérapie en 2008, l'activité en 2009 s'est élevée à 532 séances de chimiothérapie réparties sur 69 patients, pour un total de 547 patients pris en charge au total. L'équipe comportait 8 salariés à temps plein fin 2009 .

Le réseau reçoit un budget de la part de l'Urcam (Union Régionale des Caisse d'Assurance Maladie) sur la dotation FIQCS (Fond d'Intervention pour la Qualité et la Coordination des Soins). Sur ce budget, le réseau doit payer l'ensemble de ses salariés, dont les infirmières qui coordonnent les soins, la préparation par une pharmacie hospitalière et la livraison des molécules de chimiothérapie. La ressource de base de l'activité du réseau est donc l'heure de coordination infirmière. Seront facturées directement à l'assurance maladie l'analyse de sang de contrôle préalable, la consultation du médecin traitant qui donnera le feu vert, les médicaments nécessaires dont les molécules de chimiothérapie, la prestation de l'infirmière qui administrera le médicament ainsi que ses frais de déplacement et le kit stérile de matériel permettant la perfusion.

2009 était la première année de plein exercice du réseau. Sur cette première année de montée en charge, le réseau a bénéficié de 31 mois équivalent temps plein d'infirmière, ce qui représente, une fois déduit les congés et les jours fériés chômés, 4065 h disponible pour la coordination des soins. Ce temps de coordination a permis de prendre en charge 547 patients tous soins confondus, dont 69 qui ont reçu en tout 532 chimiothérapies à domicile, soit une moyenne de 7,7 chimiothérapies par patient.

Le bilan financier fait état d'un total de dépenses de fonctionnement de $489466 €$. Sur ces dépenses, une première somme (49 $864 €$ ) peut être directement affectée aux chimiothérapies: le transport des médicaments (assuré uniquement pour molécules de chimiothérapies, les autres médicaments étant fournis par la pharmacie de proximité du patient qui va les récupérer lui-même) et la fabrication de la molécule de chimiothérapie. Une 
seconde somme (72 $683 €$ ) peut être considérée comme une charge à affecter à une sousactivité. La structure de direction du réseau est calibrée pour supporter une montée en charge et la direction du réseau estime que ce niveau de charge devrait supporter une activité double de celle de 2009. Aussi avons-nous utilisé, pour les charges de structures qui répondait à ce critère, un coefficient d'imputation rationnelle de 0,5. Enfin, la permanence médicale oncologique, ainsi que les locaux, qui sont actuellement fourni gracieusement par le Centre Léon Bérard, constituent-ils des charges supplétives de $22708 €$ qu'il convient d'ajouter.

\section{Tableau 7 : Coût d'une heure de coordination}

\begin{tabular}{|lr|}
\hline Total des dépenses comptables & $489466 €$ \\
Charges directement liées aux & \\
chimiothérapies & $-49864 €$ \\
Charges de direction soumise à & $-72683 €$ \\
imputation rationelle & $22708 €$ \\
Charges supplétives & $389627 €$ \\
Total des charges à répartir sur & $4065 \mathrm{~h}$ \\
'ensemble des soins $(\mathrm{A})$ & $95,85 €$ \\
Heures de coordination infirmière $(\mathrm{B})$ & \\
Coût d'une heure de coordination $(\mathrm{A}) /(\mathrm{B})$ &
\end{tabular}

L'heure de coordination infirmière a donc été « produite » à un coût complet de pleine activité de $95,85 €$.

La montée en charge se poursuit en 2010. Un effectif total de 4 infirmières, dont certaines à temps partiel, donne une disponibilité totale de 41 mois équivalent temps plein, qui permettent une présence moyenne de 2,5 infirmières par jour d'ouverture. Sur la base d'un mix des soins équivalent à 2009, cela devrait permettre de prendre en charge 724 patients tous soins confondus, dont 91 qui pourront recevoir en tout 704 chimiothérapies à domicile. Cette hypothèse de constance du mix patient et de proportionnalité des volumes de prise en charge va nous permettre d'estimer le coût complet de coordination d'une séance de chimiothérapie organisée par le réseau.

Les infirmières du réseau ont participé à l'élaboration d'un standard des temps passés. Ont été identifiés dans ce travail les temps passés associés à différents inducteurs que sont les patients pris en charge, le processus de gestion des chimiothérapies, les patients pris en charge pour les chimiothérapies, les séances de chimiothérapies. 
Tableau 8: Inventaire des temps passés à affecter aux chimiothérapies

\begin{tabular}{|c|c|c|c|c|c|c|}
\hline & & & & & & \\
\hline & & & \begin{tabular}{|c|} 
Pour \\
l'organisatio \\
n générale \\
des soins
\end{tabular} & \begin{tabular}{|c|} 
Pour \\
l'organisation \\
générale des \\
chimiothérapie \\
$\mathbf{s}$
\end{tabular} & $\begin{array}{c}\text { Par patient } \\
\text { recevant des } \\
\text { chimiothérapie }\end{array}$ & $\begin{array}{c}\text { Par séance de } \\
\text { chimiothérapi } \\
\text { e }\end{array}$ \\
\hline $\begin{array}{l}\text { Chaque fois qu'un nouveau patient est } \\
\text { pris en charge pour des } \\
\text { chimiothérapies à domicile }\end{array}$ & $65 \mathrm{mn}$ & $1,08 \mathrm{~h}$ & & & $1,08 \mathrm{~h}$ & \\
\hline A chaque séance de chim othérapie & $60 \mathrm{mn}$ & $0,33 \mathrm{~h}$ & & & & $0,33 \mathrm{~h}$ \\
\hline $\begin{array}{l}\text { A chaque incident pendant la séance, } \\
\text { avec une fréquence en } 2009 \text { de } 10 \\
\text { incident pour } 532 \text { chimiothérapies, } \\
\text { soit pour } 2010,10 / 532^{*} 704=13,23 \\
\text { incidents }\end{array}$ & $20 \mathrm{mn}$ & $0,33 \mathrm{~h}$ & & $4,41 \mathrm{~h}$ & & \\
\hline $\begin{array}{l}\text { Chaque semaine, pour l'organisation } \\
\text { générale des chimiothérapies }\end{array}$ & $40 \mathrm{mn}$ & $0,67 \mathrm{~h}$ & & $34,67 \mathrm{~h}$ & & \\
\hline $\begin{array}{l}\text { Par an, pour le fonctionnement } \\
\text { général de l'équipe de coordination }\end{array}$ & & $889,08 \mathrm{~h}$ & $889,08 \mathrm{~h}$ & & & \\
\hline TOTAL PAR CATEGORIE & & & $889,08 \mathrm{~h}$ & $39,08 \mathrm{~h}$ & $1,08 \mathrm{~h}$ & $0,33 \mathrm{~h}$ \\
\hline \multirow[b]{2}{*}{ Base de la mutualisation } & & & 724 patients & 704 séances & 7,7 séance/Pat & \\
\hline & & & séance/Pat & & & \\
\hline Coefficient de mutualisation & & & 0,000179 & 0,0014 & 0,1297 & 1,0000 \\
\hline Temps réparti par chimiothérapie & & $0,6887 \mathrm{~h}$ & $0,1594 \mathrm{~h}$ & $0,0555 \mathrm{~h}$ & $0,1405 \mathrm{~h}$ & $0,3333 \mathrm{~h}$ \\
\hline
\end{tabular}

La coordination d'une chimiothérapie à domicile en réseau est réalisée à un coût moyen estimé à $66,01 €(0,6887 \mathrm{~h} \times 95,85 €)$.

\section{Tableau 9: Coût complet d'une chimiothérapie réalisée en réseau}

\begin{tabular}{|c|c|c|c|}
\hline Coûts de coordination & Chimio courte & $\begin{array}{l}\text { Chimio longue } \\
\text { (1h30 max) }\end{array}$ & $\begin{array}{c}\text { Herceptine } \\
\text { (pas de feu } \\
\text { vert } \\
\text { médecin } \\
\text { nécessaire) }\end{array}$ \\
\hline $\begin{array}{l}\text { organisation, coordination, mise à jour } \\
\text { dossier patient } \\
\text { Coûts directs }\end{array}$ & $66,01 €$ & $66,01 €$ & $66,01 €$ \\
\hline 1. Préparation de la chimiothérapie & $35,00 €$ & $35,00 €$ & $35,00 €$ \\
\hline 2. Livraison de la chimiothérapie & $27,72 €$ & $27,72 €$ & $27,72 €$ \\
\hline 3. Feu vert médecin & $23,00 €$ & $23,00 €$ & \\
\hline 4. Injection par l'infirmière & $4,73 €$ & $47,25 €$ & $31,50 €$ \\
\hline $\begin{array}{l}\text { 5. Déplacement IDE (habite au maximum à } \\
10 \mathrm{~km} \text { du patient) }\end{array}$ & $12,30 €$ & $12,30 €$ & $12,30 €$ \\
\hline 6. Kit de perfusion & $15,35 €$ & $15,35 €$ & $15,35 €$ \\
\hline COUT TOTAL POUR UNE CHIMIO & $184,11 €$ & $226,63 €$ & $187,88 €$ \\
\hline
\end{tabular}

Le transport des chimiothérapies a été estimé, sur la base d'une étude réalisée sur les six premiers mois de fonctionnement du service de livraison du réseau, à 27,72€ par livraison en moyenne. La préparation de chimiothérapie est facturée par la PUI $35 €$ par chimiothérapie. L'injection par l'infirmière varie en fonction de la durée d'administration de la molécule. Le 
feu vert d'un médecin est nécessaire quelle que soit la molécule, sauf l'Herceptine. Le coût complet d'une chimiothérapie pour l'assurance maladie varie donc, selon notre estimation, de $174,38 €$ à $228,38 €$ hors molécule.

\subsection{L’analyse de l'échelle nationale des coûts hospitaliers}

Pour rendre les données comparables, nous avons utilisé l'Echelle Nationale de Coût 2007 mise en ligne par l'ATIH. L'Echelle Nationale de coûts disponible sur le site de l'Agence Technique de l'Information Hospitalière présente une estimation des coûts des séjours hospitaliers par groupe homogène de malade. L'Etude Nationale des Coûts a été constituée à partir des données de coûts collectées par 55 établissements ex-DGF (secteur public et PSPH) et 44 établissements ex-OQN (secteur privé) et jugées de qualité suffisante.

Le coût complet est obtenu après plusieurs corrections qui permettent d'homogénéiser les conditions de calcul nationales : (1) abattements liés aux MERRI (mission d'enseignement et de recherche) qui conduisent de fait à allouer entre 9 et $17 \%$ de ressources supplémentaires à certains établissements (2) neutralisation du coefficient géographique, qui conduit à allouer plus de ressources à certaines zones (environs $7 \%$ en île de France) (3) élimination des séjours extrêmes en durée et en coût.

Lorsque le nombre de séjour est supérieur à 30, le coût moyen affiché est la moyenne des établissements sélectionnés. Le coût complet peut varier d'un établissement à l'autre en fonction des techniques employées. Le calcul d'un intervalle de confiance permet de situer que le coût réel a statistiquement $95 \%$ de chance de se trouver dans l'intervalle entre une borne haute et une borne basse ce qui permet de donner une indication sur la précision de la moyenne ainsi calculée.

Dans le cas des séjours ambulatoires de chimiothérapie que nous étudions, le nombre de séjours analysés : 207766 pour le secteur public et PSPH (20,43\% des séjours) et 89721 pour le secteur privé (12,69\% des séjours). La moyenne du coût du GHM pour le secteur ex DGF se situe à $1021,70 €$ avec un intervalle de confiance situé entre 969,68 $€$ et 1 073,70€. Soit une variation de 5\% autour du coût moyen. Cette estimation est donc assez précise. La moyenne du coût du GHM pour le secteur ex OQN se situe à $886,17 €$ avec un intervalle de confiance situé entre $658,71 €$ et 1 182,56 $€$. Soit une variation de $33,45 \%$ autour du coût moyen. Cette estimation est donc beaucoup moins précise. 


\section{Tableau 10: Analyse de l'Echelle Nationale de coûts pour les séjours hospitaliers [28Z07Z chimiothérapie en séance]}

\begin{tabular}{|c|c|c|c|c|c|}
\hline Postes de coûts coût complet & $\begin{array}{c}\text { Public et } \\
\text { PSPH }\end{array}$ & Privé & Inclus & Exclus & Commentaires \\
\hline Amortissement location clinique (hors SI SC réa) & $1,22 €$ & $1,49 €$ & $\mathrm{X}$ & & \multirow{5}{*}{$\begin{array}{l}\text { Un partie des coûts cliniques est déclenchée par d'autres soins } \\
\text { que la chimiothérapie, aussi conviendrait-il de retraiter ce poste } \\
\text { avant comparaison pour estimer la part de la seule } \\
\text { chimiothérapie. Dans le cadre d'un réseau de soins, toute autre } \\
\text { prestation sera tarifée à part et représentera un coût } \\
\text { supplémentaire. }\end{array}$} \\
\hline Entretien maintenance clinique (hors SI SC réa) & $0,34 €$ & $0,32 €$ & $\mathrm{X}$ & & \\
\hline Personnel autre clinique (hors SI SC réa) & $24,77 €$ & $4,93 €$ & $\mathrm{X}$ & & \\
\hline Personnel médical clinique (hors SI SC réa) & $32,38 €$ & $0,00 €$ & $\mathrm{X}$ & & \\
\hline Personnel soignant clinique (hors SI SC réa) & $73,34 €$ & $32,07 €$ & $\mathrm{X}$ & & \\
\hline Dépenses de Surveillance Continue & $0,06 €$ & $0,00 €$ & & $\mathrm{X}$ & \multirow{3}{*}{$\begin{array}{l}\text { Le transfert d'un patient en réanimation ou dans un service de } \\
\text { soins intensif serait facturé en sus pour une prise en charge en } \\
\text { réseau. }\end{array}$} \\
\hline Dépenses Soins Intensifs & $0,10 €$ & $0,00 €$ & & $\mathrm{X}$ & \\
\hline Dépenses Réanimation & $0,00 €$ & $0,00 €$ & & $\mathrm{X}$ & \\
\hline Dépenses totales Anesthésie & $0,23 €$ & $0,00 €$ & & $\mathrm{X}$ & \multirow{10}{*}{$\begin{array}{l}\text { Les dépenses correspondantes attestent de la réalisation d'autres } \\
\text { actes que la chimiothérapie, qui dans le cadre du réseau seraient } \\
\text { facturées en sus. Elles sont donc exclues pour la comparaison. }\end{array}$} \\
\hline Dépenses totales Bloc & $1,22 €$ & $0,10 €$ & & $\mathrm{X}$ & \\
\hline Dépenses totales Bloc Obstétrique & $0,00 €$ & $0,00 €$ & & $\mathrm{X}$ & \\
\hline Dépenses totales Dialyse & $0,00 €$ & $0,00 €$ & & $\mathrm{X}$ & \\
\hline Dépenses totales Exploration fonctionnelle & $1,28 €$ & $0,00 €$ & & $\mathrm{X}$ & \\
\hline Dépenses totales Imagerie & $5,18 €$ & $0,01 €$ & & $\mathrm{X}$ & \\
\hline Dépenses totales Imagerie interventionnelle & $0,04 €$ & $0,00 €$ & & $\mathrm{X}$ & \\
\hline Dépenses totales Laboratoire & $6,39 €$ & $0,00 €$ & & $\mathrm{X}$ & \\
\hline Dépenses totales Laboratoire Hors nomenclature & $1,25 €$ & $0,00 €$ & & $\mathrm{X}$ & \\
\hline Dépenses totales Radiothérapie & $1,60 €$ & $0,00 €$ & & $\mathrm{X}$ & \\
\hline Dépenses totales SMUR terrestre & $0,03 €$ & $0,00 €$ & & $\mathrm{X}$ & \multirow{3}{*}{$\begin{array}{l}\text { Les dépenses de SMUR et d'Urgence représenteraient pour le } \\
\text { réseau, l'utilisation de structures de repli. Elles sont exclus de } \\
\text { notre comparaison. }\end{array}$} \\
\hline Dépenses totales SMUR aérien & $0,00 €$ & $0,00 €$ & & $\mathrm{X}$ & \\
\hline Dépenses totales Urgences & $0,01 €$ & $0,01 €$ & & $\mathrm{X}$ & \\
\hline Dépenses totales Autres Medico-technique & $0,42 €$ & $0,00 €$ & & $\mathrm{X}$ & Seraient facturées en sus dans la prise en charge en réseau \\
\hline L GG (Logistique Générale) Blanchisserie & $3,20 €$ & $2,88 €$ & $\mathrm{X}$ & & \multirow{9}{*}{$\begin{array}{l}\text { Un partie des coût de logistique générale et médicale sont } \\
\text { déclenchés par d'autres soins que la chimiothérapie, aussi } \\
\text { conviendrait-il de retraiter ce poste avant comparaison pour } \\
\text { estimer la part de la seule chimiothérapie. Dans le cadre d'un } \\
\text { réseau de soins, toute autre prestation sera tarifée à part et } \\
\text { représentera un coût supplémentaire. }\end{array}$} \\
\hline LGG Restauration & $4,85 €$ & $4,41 €$ & $\mathrm{X}$ & & \\
\hline LGG Accueil et gestion des malades & $5,02 €$ & $6,32 €$ & $\mathrm{X}$ & & \\
\hline LGG Services administratifs à caractère général & $24,77 €$ & $25,68 €$ & $\mathrm{X}$ & & \\
\hline LGG Services administratifs liés au personnel & $10,87 €$ & $1,44 €$ & $\mathrm{X}$ & & \\
\hline LGG DIM & $3,04 €$ & $1,78 €$ & $\mathrm{X}$ & & \\
\hline LGG DSIO & $7,43 €$ & $2,69 €$ & $\mathrm{X}$ & & \\
\hline LGG Entretien maintenance & $9,91 €$ & $7,95 €$ & $\mathrm{X}$ & & \\
\hline LGG Services hôteliers & $17,01 €$ & $9,57 €$ & $\mathrm{X}$ & & \\
\hline Do Bralleardage et transpont pedestre des & $2,50 €$ & $1,09 €$ & & $\mathrm{X}$ & La moyenne des Indices de Karnosky en reseau est de 80. Le \\
\hline LGG Transport motorisé des patients (hors SMUR) & $1,72 €$ & $0,00 €$ & & $\mathrm{X}$ & $\begin{array}{l}\text { patient est donc autonome. Ioute autre examen necessitant } \\
\text { dénlacement serait facturé en sus. }\end{array}$ \\
\hline LM (Logistique Médicale) Pharmacie & $49,09 €$ & $26,72 €$ & $\mathrm{X}$ & & \multirow{6}{*}{$\begin{array}{l}\text { Une partie des coûts correspondants peuvent être générés par } \\
\text { d'autres soins pris en charge en même temps que la } \\
\text { chimiothérapie, aussi conviendrait-il de retraiter les coûts pour } \\
\text { en tenir compte. }\end{array}$} \\
\hline LM Stérilisation & $0,76 €$ & $0,04 €$ & $\mathrm{X}$ & & \\
\hline LM Génie Biomédical & $0,46 €$ & $0,08 €$ & $\mathrm{X}$ & & \\
\hline LM Hygiène et Vigilance & $7,36 €$ & $1,88 €$ & $\mathrm{X}$ & & \\
\hline Autre LM & $0,80 €$ & $0,00 €$ & $\mathrm{X}$ & & \\
\hline Coûts Directs (CD) Autres consommables & $12,82 €$ & $7,95 €$ & $\mathrm{X}$ & & \\
\hline CD DMI non facturables en sus & $0,84 €$ & $0,19 €$ & & $\mathrm{X}$ & \multirow{4}{*}{ Seraient facturées en sus dans la prise en charge en réseau } \\
\hline CD DMI facturables en sus & $0,20 €$ & $0,05 €$ & & $\mathrm{X}$ & \\
\hline CD Médicaments ATU & $5,68 €$ & $0,00 €$ & & $\mathrm{X}$ & \\
\hline CD Sang & $6,49 €$ & $0,93 €$ & & $\mathrm{X}$ & \\
\hline $\begin{array}{l}\text { CD Spécialités pharmaceutiques non facturables en } \\
\text { sus }\end{array}$ & $24,16 €$ & $22,32 €$ & & $\mathrm{X}$ & \multirow{2}{*}{$\begin{array}{l}\text { Toutes les molécules seraient facturées en sus dans la prise en } \\
\text { charge en réseau }\end{array}$} \\
\hline CD Spécialités pharmaceutiques facturables en sus & $668,41 €$ & $721,07 €$ & & $\mathrm{X}$ & \\
\hline CD Sous-traitance SMUR & $0,03 €$ & $0,00 €$ & & $\mathrm{X}$ & Non pris en compte en l'état (voir SMUR ci-avant) \\
\hline CD Sous-traitance Imagerie & $0,26 €$ & $0,00 €$ & & $\mathrm{X}$ & \multirow{13}{*}{ Seraient facturées en sus dans la prise en charge en réseau } \\
\hline CD Sous-traitance Laboratoire & $2,19 €$ & $0,00 €$ & & $\mathrm{X}$ & \\
\hline CD Sous-traitance Laboratoire Hors nomenclature & $0,62 €$ & $0,00 €$ & & $\mathrm{X}$ & \\
\hline CD Sous-traitance Hospitalisation à l'extérieur & $0,01 €$ & $0,09 €$ & & $\mathrm{X}$ & \\
\hline CD Sous-traitance Autre & $0,68 €$ & $2,11 €$ & & $\mathrm{X}$ & \\
\hline CD Honoraires des $\mathrm{PH}$ & $0,15 €$ & & & $\mathrm{X}$ & \\
\hline CD Rémunération à l'acte du personnel & $0,52 €$ & & & $\mathrm{X}$ & \\
\hline $\begin{array}{l}\text { Honoraires médicaux - Laboratoire d'anatomie } \\
\text { pathologie }\end{array}$ & & $0,01 €$ & & $\mathrm{X}$ & \\
\hline Honoraires médicaux - Laboratoires autres & & $0,67 €$ & & $\mathrm{X}$ & \\
\hline Honoraires médicaux - Imagerie & & $0,33 €$ & & $\mathrm{X}$ & \\
\hline Honoraires médicaux - Anesthésie & & $0,00 €$ & & $\mathrm{X}$ & \\
\hline Honoraires médicaux - Obstétrique & & $0,00 €$ & & $\mathrm{X}$ & \\
\hline Honoraires médicaux - Chirurgie & & $0,02 €$ & & $\mathrm{X}$ & \\
\hline Honoraires médicaux - Autres actes médicaux & & $29,94 €$ & $\mathrm{X}$ & & $\begin{array}{l}\text { PrIs en comple a nauteur de zJE raentme dans la structure } \\
\text { tarifaire }\end{array}$ \\
\hline Honoraires soignants & & $0,02 €$ & & $\mathrm{X}$ & \multirow{2}{*}{ Seraient facturées en sus dans la prise en charge en réseau } \\
\hline Honoraires autres & & $0,00 €$ & & $\mathrm{X}$ & \\
\hline Structure Immobilier & $7,12 €$ & $2,79 €$ & $\mathrm{X}$ & & \multirow{2}{*}{$\begin{array}{l}\text { Pris en compte au titre des couts Immobiliers et des besoins } \\
\text { financiers liés, pour partie liés aux autres soins prodigués dans } \\
\text { les mêmes séiours }\end{array}$} \\
\hline Structure financier & $16,97 €$ & $28,79 €$ & $\mathrm{X}$ & & \\
\hline
\end{tabular}


Le coût complet est présenté hors frais de structure, défini dans l'ENC en deux postes de dépenses. D’une part, les frais immobiliers sont constitués par les charges liées au patrimoine immobilier des établissements : charges locatives et de copropriétés, entretien et réparation des biens immobiliers, taxe foncière et autres impôts locaux, dotations aux amortissements des constructions, des agencements et aménagements de terrains. D'autre part, les frais financiers constitués par les charges financières incorporables dans l'ENC : intérêts des emprunts et dettes, intérêts des crédits bail retraités et intérêts des comptes courants créditeurs.

Dans la perspective de la comparaison des coûts de réalisation de la chimiothérapie en Etablissement de Santé versus un réseau de santé, il convient de retraiter cette base des coûts hospitaliers. Certaines charges sont liées à des prestations qui, si elles devaient être organisées par le réseau de santé, viendrait augmenter les coûts de la prise en charge. Nous allons donc détailler le retraitement poste par poste et les raisons de nos choix dans le tableau ci-après.

Après avoir déterminé quels étaient les postes de dépenses qui permettaient de rendre comparables la chimiothérapie réalisée en réseau et la même prestation réalisée en hôpital de jour, il nous a fallu estimer ce que pouvait représenter les autres soins dans cette base de coûts. Nous nous sommes livrés à une approximation à partir des données disponibles sur les bases de l'ATIH. Nous avons utilisé le dénombrement de l'intégralité des actes enregistrés dans les séjours de la base pour l'année 2007, année qui a servi de base à l'ENC, et qui n'étaient pas des injections de produits anticancéreux. Les actes enregistrés ne sont sans doute pas exhaustif, mais cela donne une première approche. Nous avons arbitrairement considéré le ratio entre actes autres que chimiothérapie et nombre de séjours $(13,80 \%$ pour le public et $2,69 \%$ pour le privé) pour corriger les postes de charge impactés par la présence d'autres actes dans le séjour.

Tableau 11 : Estimation corrigée du coût de la chimiothérapie en Etablissement Hospitalier

\begin{tabular}{|c|c|c|c|c|c|c|}
\hline \multirow{2}{*}{ Fostes de coîts coît complet } & \multirow{2}{*}{$\underset{\text { FubFH }}{\text { Furic et }}$} & \multirow{2}{*}{ Frivé } & \multicolumn{2}{|c|}{$\begin{array}{c}\text { Coefificint de } \\
\text { prise en campte }\end{array}$} & \multirow{2}{*}{$\begin{array}{c}\text { Fublic et } \\
\text { PSFH }\end{array}$} & \multirow{2}{*}{ Frivé } \\
\hline & & & $\underset{\text { Fubsic }}{\text { et PSPH }}$ & Frivé & & \\
\hline 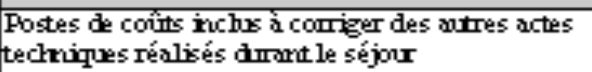 & $313,54 €$ & $109,79 €$ & $8169 \%$ & $97,10 \%$ & $256,13 €$ & $164,87 €$ \\
\hline Hanasires méticanx - intres actes méticaux & & $2994 €$ & & $23,00 €$ & & $23,00 €$ \\
\hline \multicolumn{5}{|l|}{ Tataldes coûtsretratiés } & $256,13 €$ & $187,87 €$ \\
\hline
\end{tabular}


La différence entre les coûts retraités structure public et structure privée est plus faible que lorsque l'on compare les tarifs, mais elle reste cependant significative. On peut alors remarqué que le coût en réseau se situe dans une fourchette inférieure aux coûts hospitaliers, mais ne couvre que des chimiothérapies courtes (Tableau 7).

Tableau 12 : Coût comparé de l'injection d'une chimiothérapie, hors molécule

\begin{tabular}{|lcl|}
\hline Réseau de soins & de $174,38 €$ à & $228,38 €$ pour une durée maximum d'1,30 heure \\
\hline Hôpital de jour public & $256,13 €$ & toute durée \\
\hline Hôpital de jour privé & $187,87 €$ & toute durée \\
\hline
\end{tabular}

Il convient pourtant de ne pas trop s'y attarder sans études plus approfondies. Les structures hospitalières accueillent par ailleurs des chimiothérapies de durées très variables, qui vont de l'injection simple à la perfusion de plusieurs heures, alors que celles réalisées en réseau ne dépassent pas une heure trente. Les CHU et les CLCC qui accueillent souvent les patients les plus complexes à traiter ont sans doute des durées plus longues que les structures privées, mais nous ne disposons pas des données nécessaires pour en estimer l'impact.

Cette estimation permet cependant de donner du sens aux comparaisons en mettant en perspective des éléments plus comparables que les données brutes. Les fourchettes de coûts sont proches et compte tenu des hypothèses et remarques formulés, les éléments analysés ne permettent pas de discriminer un mode d'administration sur la base de son coût de fonctionnement. Bien que cela ne soit pas parfaitement démontré, il semblerait qu'à soins et durée égale, les coûts d'administration soient relativement proches.

Il reste cependant un élément non négligeable du coût des chimiothérapies pour être complet sur l'analyse comparée, celui du transport des patients.

\subsection{Le problème particulier du transport des patients}

Le coût de transport des patients est une donnée extrêmement variable en fonction de l'éloignement de ces derniers du lieu où la chimiothérapie doit leur être administrée, et du moyen de transport qu'ils vont utiliser. Ils peuvent bénéficier d'un transport sanitaire, qui sera pris en charge par la sécurité sociale lorsqu'il est prescrit par un médecin. Le cancer est considéré comme une affection longue maladie, et les patients atteints de cancer sont pris en charge à $100 \%$. Les centres de traitement des cancers sont des centres urbains, et la cancérologie va être un consommateur important de transports sanitaires pour acheminer les 
patients jusqu'aux centres de traitement.

Nous allons utiliser la grille de remboursement de 1'assurance maladie pour intégrer les transports à notre raisonnement. Les remboursements admis par la sécurité sociale pour les trois solutions admises sont présentées dans le tableau 8.

Tableau 13: Coût de transport des patients, données URCAM

\begin{tabular}{|l|r|r|r|}
\cline { 2 - 4 } \multicolumn{1}{c|}{} & \multicolumn{1}{c|}{ Fixe } & \multicolumn{1}{c|}{ Variable } \\
\hline & $\begin{array}{c}\text { Fixe } \\
\text { minimum }\end{array}$ & $\begin{array}{c}\text { Fixe } \\
\text { maximum }\end{array}$ & $\begin{array}{c}\text { Prix du km } \\
\text { (max })\end{array}$ \\
\hline Sans prise en charge & $0,00 €$ & $0,00 €$ & $0,35 €$ \\
\hline Voiture personnelle & $11,48 €$ & $13,28 €$ & $0,83 €$ \\
\hline VSL & $51,30 €$ & $57,37 €$ & $2,12 €$ \\
\hline Ambulance & \multicolumn{2}{c}{} \\
\hline
\end{tabular}

L'administration de la chimiothérapie nécessite soit que le patient se rende dans un hôpital de jour, soit que l'on achemine la (ou les) molécules de chimiothérapie chez lui, pour que l'infirmière libérale de proximité puisse lui les administrer.

Le schéma ci-après présente nos différentes alternatives pour la chimiothérapie ambulatoire, assorties ou non de coûts de transport selon les différentes modalités possibles et en fonction de la distance qui sépare le patient et la structure de soin hospitalière. Pour permettre cette simulation, le coût de transport des chimiothérapies organisées par le réseau, qui avait été calculé de manière forfaitaire a été rendu variable sur la base des kilomètres qui ont nécessaires à la livraison des patients en 2009. Les dépenses totales se montaient à $31160 €$ pour $44796 \mathrm{~km}$ environ, soit $0,70 €$ par $\mathrm{km}$. Ce coût au kilomètre tient compte de la mutualisation permise par l'organisation de tournée et les livraisons groupées (une livraison par semaine lorsqu'il y a plusieurs chimio dans la même semaine). Nous n'avons pas retenu d'hypothèse de mutualisation concernant le transport du patient.

A ce jour, les chimiothérapies étant préparées dans les pharmacies hospitalières, la distance à parcourir par le patient pour aller de chez lui à l'hôpital est la même que la distance que doit parcourir la chimiothérapie pour aller de l'hôpital a chez lui. La figure 1 présente les coûts des différentes alternatives présentées ci-avant, en fonction de la distance entre le patient et la structure de soins, qui à ce jour est également le lieu de préparation de la molécule administrée à domicile. 
Figure 7 : Simulation du coût pour l'assurance maladie des solutions organisationnelles en fonction de l'éloignement des patients

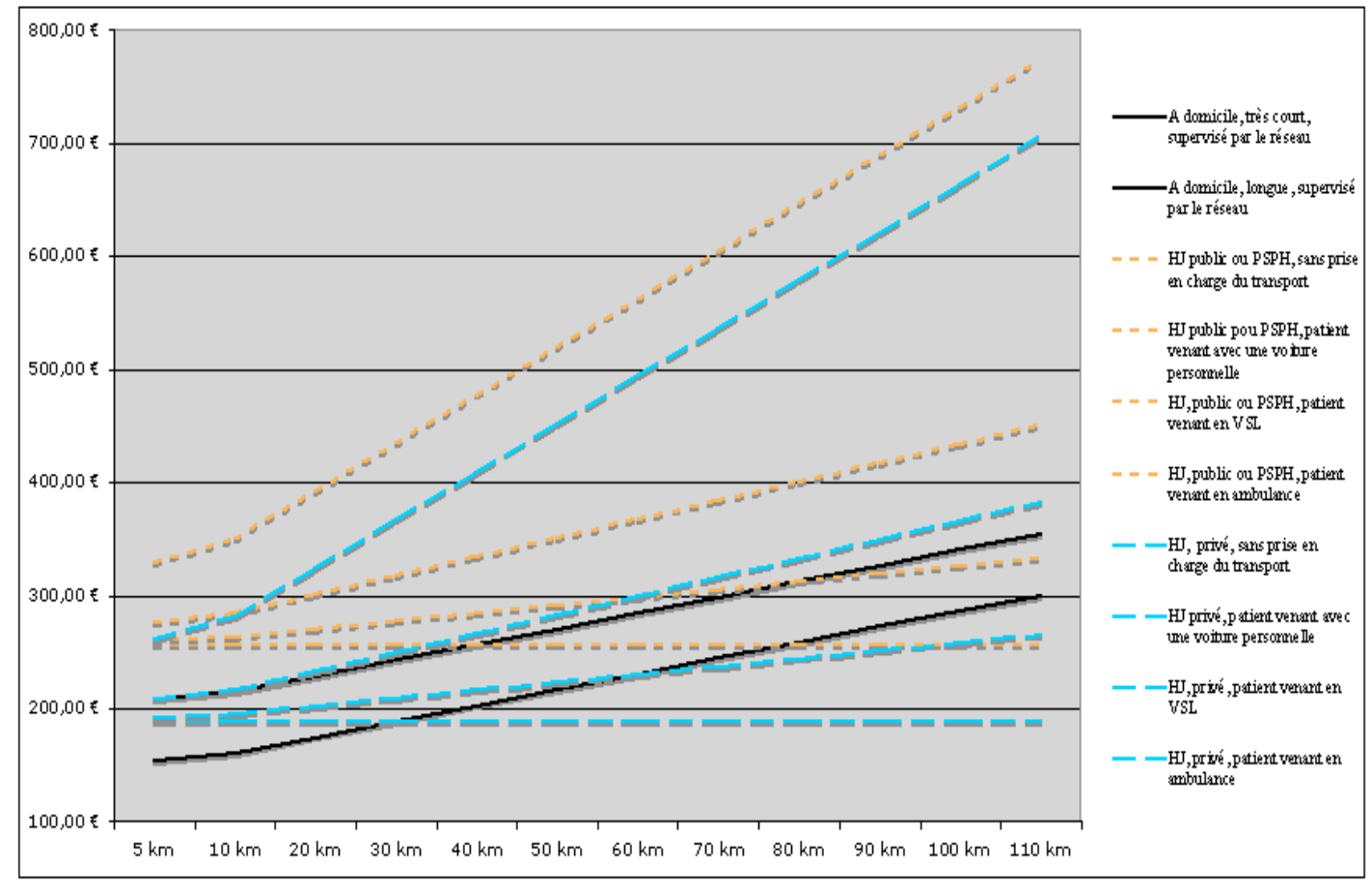

Cette simulation met en évidence de façon claire l'impact des coûts de transport sur le coût global de la séance de chimiothérapie. Sur notre schéma, on peut par exemple lire que lorsque la séance consiste en une injection et que le patient réside à moins d'environ $30 \mathrm{~km}$ d'une structure privée qui peut le prendre en charge, alors le réseau est moins coûteux. S'il habite à plus de $30 \mathrm{~km}$ et si son déplacement ne fait pas l'objet d'une prise en charge financière, une structure hospitalière privée peut être moins coûteuse. Cette dernière sera moins coûteuse si la chimiothérapie dure 1 heure et demi, que le patient habite à $40 \mathrm{~km}$ vient avec son véhicule personnel, et ce même s'il demande un remboursement kilométrique. Enfin, lorsque le patient utilise une ambulance pour recevoir une simple chimiothérapie, le réseau devient bien plus performant que toute autre solution.

Notre simulation montre la sensibilité de la fonction de coût à un paramètre qui n'est pas médical, mais souligne également qu'il n'existe pas une réponse unique quant à la solution la plus intéressante pour l'assurance maladie. Cette dernière est fonction de l'éloignement du patient, de la durée de la séance, mais également de la possibilité d'organiser le même jour des actes qui, ne pouvant se dérouler à domicile comme la radiothérapie ou les examens de radiologie, mutualiseront le frais de transport. 
Ce chiffrage montre que l'argument des coûts de transport recouvre une certaine réalité, mais ne doit pas masqué que ce paramètre est sans doute «surreprésenté » dans l'esprit des tenants des soins à domicile. Tous d'abord tous les patients n'utilisent pas des transports sanitaires d'une part et d'autre part tous les patients ne résident pas à plus de $40 \mathrm{~km}$ de leur lieu de soins. L'étude infirmière montre que la pratique de la chimiothérapie n'est pas plus importante en zone rurale, loin des établissements de santé, qu'en zone urbaines, à proximité de ceux-ci (cf annexe 2). La possibilité de réaliser des soins concomitant est elle sur-représentée dans l'esprit des défenseurs de l'hôpital de jour. 


\section{Troisième Partie : Analyse et discussion}

Notre analyse des données de coût montre que la solution à domicile n'est pas forcément plus coûteuse qu'une solution en structure hospitalière, particulièrement lorsque le patient est éloigné géographiquement. D'autant qu'il serait possible dans l'avenir, si le patient est très éloigné du lieu de soin, de trouver une pharmacie hospitalières plus proche de son domicile qui pourrait préparer les molécules, réduisant ainsi les kilomètre à parcourir par les livreurs. Les infirmières libérales, sur la zone géographique où nous les avons interrogées, sont prêtes à prendre en charge plus de chimiothérapies à domicile. Les conditions de réalisation sont suffisamment encadrées pour que la prestation se réalise dans des conditions de qualité et de sécurité suffisante.

Pourtant, il n'est pas certain que les conditions actuelles permettent véritablement le développement de ces alternatives à domicile. L'analyse des modèles d'activité des acteurs est susceptible de montrer comment s'agencent les éléments que nous avons identifié dans les différents espaces de décision des acteurs de soins.

\section{1. la réglementation : un problème surmontable à ce jour}

Le fait que la chimiothérapie à domicile n'augmente pas réellement ces dernières années est également mis en cause par les contraintes des cadres réglementaires. « En France, le cadre réglementaire de la T2A doit tenir compte d'autres cadres réglementaires comme ceux du médicament définis par la HAS mais aussi par l'Afssaps, du règlement lié à l'hospitalisation (tenant compte de la sécurité hospitalière, du fonctionnement, du financement de l'établissement hospitalier) et de l'activité de la médecine de ville. L'accumulation de ces cadres réglementaires spécifiques accroît les difficultés notamment dans le champ de l'Oncologie et en particulier de la prise en charge à domicile » note le Dr Michèle Brami [Le Nouveau Cancérologue]. La différence d'exigences entre le domicile et l'hôpital s'avère donc être une difficulté rencontrée par de nombreux praticiens. Le fait de pratiquer des chimiothérapies à distances semble engendrer de nombreuses contraintes d'organisation, de temps nécessaire, de responsabilité... .

Le problème du cadre réglementaire et la lourdeur qu'il engendre pourrait-elle expliquer la faiblesse des «entrepreneurs» pour mettre en place des réseaux de soins? Car si un cancérologue souhaite permettre à son patient d'accéder à une prise en charge à domicile, 
encore faut-il qu'il puisse trouver une structure pour organiser cette prise en charge. Ceci est vrai à la mise en place de l'organisation de la chimiothérapie à domicile, qui est un parcours du combattant pour des réseaux sensés être portés par des libéraux peu rompus à ce type de complexité. Ceci explique pourquoi ce sont souvent à partir des structures hospitalières que ces réseaux susceptibles de prendre en charge des chimiothérapies se sont développées, les entrepreneurs « hospitaliers » étant mieux dotés pour franchir les obstacles.

Mais une fois cette difficulté surmontée, cela n'explique pas que cette prestation ne se développe pas véritablement dans des proportions plus importantes.

\section{Des professionnels de santé disponibles en dehors de l'hôpital}

De nombreuses infirmières libérales ont reçu l'accréditation de chimiothérapie nécessaires à la prise en charge des chimiothérapies intraveineuses à domicile. Plusieurs déplorent que la formation qu'elle ont suivie pour cela ne soit pas suivie de plus de sollicitations. Pour celle dont le diplôme est trop ancien pour avoir reçu la formation à la chimiothérapie, la formation est un investissement qui n'est pas suivi d'un amortissement suffisant.

A l'extérieur de l'hôpital, les infirmières ne sont pas les seules à vouloir être impliquées dans ces soins. Dans les années 90, lorsque les HAD telles qu'elles existent aujourd'hui se sont mises en place et dotées de médecins, prendre en charge des chimiothérapies étaient un moyen de montrer un positionnement de la structure dans une activité médicale hospitalière. Par la suite, l'absence de réseau territorial de soins en cancérologie sur certaines zones géographiques, comme c'est le cas sur les départements de la Savoie, de la Haute Savoie, ou encore le développement d'un réseau de soins exclusivement orienté sur les soins palliatifs, comme c'est le cas dans le département de la Loire, a amené les HAD à intervenir sur ce créneau de la chimiothérapie à domicile. Aujourd'hui, sur le département du Rhône, l'existence d'un réseau territorial de cancérologie et d'une HAD généraliste «historique » crée quelques petites frictions de concurrence, alors qu'elle ne devraient théoriquement pas se trouver en concurrence sur les mêmes patients.

Les médecins libéraux, qui dans le cadre de la chimiothérapie sont mobilisés pour donner le feu vert à l'administration des cures, sont également intéressés par cette possibilité qui leur est offerte de ne pas perdre le contact avec des patients qu'ils ont suivi souvent pendant des années, et qui pendant le traitement anticancéreux «disparaissent» dans les couloirs hospitaliers, pour ne réapparaître que dans les épisodes inter-cures, sans qu'ils soient toujours au courant de leur traitement et de leur dossier hospitalier. Lorsque le traitement est un échec, 
les aspects médicaux de la fin de vie sont renvoyés sur le généraliste, qui à défaut d'être présent en continu sur la prise en charge du patient, a le sentiment de ne faire que le «sale boulot ».

«Monter» en soins techniques est une revendication que nous avons beaucoup entendu à la fois chez les libéraux, ou encore chez les représentants de certains hôpitaux « locaux », qui se verraient bien prendre le relais des structures hospitalières urbaines très techniques pour ce genre de prestations. L'idée qu'ils ne souhaitent pas être confinés aux activités que ces hôpitaux ne veulent pas prendre en charge est assez fréquemment exprimée.

Cependant, si les expériences actuelles sont volontiers prises en relais par les infirmières libérales et certains médecins généralistes, il n'est pas certain que le personnel soit uniformément disponible sur tout le territoire. Dans notre étude sur les HAD, il est apparu clairement que le recours aux infirmières libérales étaient difficile en raison de leur saturation sur certaines zones géographiques. La Région Rhône-Alpes est pour l'essentiel assez bien dotée en personnel soignant, ce qui n'est pas le cas dans certaines régions où le relais ne peut être pris pas un personnel soignant déjà trop peu nombreux pour assurer les opérations qui lui sont déjà dévolues.

\section{La cancérologie, une activité hospitalière}

Contrairement à d'autres soins comme la transfusion sanguine, que les acteurs hospitaliers de la cancérologie délégueraient volontiers à d'autres (Buthion 2009), la chimiothérapie fait partie du cœur de métier de la cancérologie. C'est un acte technique marqueur d'une activité oncologique, et à ce titre, elle appartient au champ que la cancérologie souhaite garder dans leur champs d'activité. Or la cancérologie est à ce jour une spécialité excerçée quasiment exclusivement dans les centres hospitaliers. Apparemment, le fait de garder la maitrise de la prescription ne leur suffit pas, la présence physique du patient en traitement dans les lieux qu'ils « maîtrisent » est tout aussi importante. Le corps professionnel des cancérologues ne se départit pas volontiers de ses patients pour les confier aux soins de ville.

Traditionnellement, l'activité hospitalière se mesure en volume de patients pris en charge. Le Dr Jean-Marie Tigaud explique que le problème fondamental est de savoir si les institutions, c'est-à-dire les hôpitaux, ont intérêt de sortir de l'activité [Le Nouveau Cancérologue]. Il cite : « notre "poids" dans l'Institution hospitalière est intimement lié à notre activité : plus on a de l'activité, plus le nombre d'infirmières et de médecins est important». Son argumentaire expose l'idée d'une non valorisation de la prescription de la chimiothérapie à domicile. 
On constate une résistance forte à « faire sortir ». L'Institut de Cancérologie de la Loire, qui possédait une autorisation pour quatre lits d'Hospitalisation à Domicile, notamment à des fins de réaliser des chimiothérapies, ne les a à ce jour jamais utilisés. Le réseau Onco69, mise en place pour prendre en charge les patients des Hospices Civils de Lyon, a renoncé à faire sortir des patients pour des chimiothérapies à domicile faute de demande de la part des cancérologues des établissements partenaires.

\section{Un financement désormais dépendant de l'activité mesurée}

Nos entretiens avec les HAD nous ont par ailleurs permis d'identifier la fluctuation de ces activités pour ces structures à domicile qui ont précédé les réseaux, et qui interviennent encore en leur absence dans la prise en charge des chimiothérapies ambulatoires à domicile. Un directeur d'HAD nous en a résumé l'historique ainsi : «Dans les années 90, les hôpitaux qui fonctionnaient en budget global avait les ressources pour réaliser les chimiothérapies, mais manquaient de personnel. Il leur arrivait donc de fournir les molécules aux structures HAD pour qu'elles les administrent à domicile à leurs patients. Ayant ouvert de nombreuses places d'hôpitaux de jour, le besoin de remplir ces structures a diminué leur intérêt pour la réalisation de ces prestations à domicile. L'arrivée de la T2A depuis 2006, et la possibilité de facturer en sus les molécules onéreuses, ont ramené les patients à l'hôpital de jour, permettant ainsi de générer de l'activité à moindre cô̂t. " La direction des Hopsices Civils de Lyon, confrontée à un déficit financier consécutif au passage à la T2A, ne fait sûrement pas de l'externalisation des soins une priorité.

Une stratégie de regroupement des soins, qui en mutualisant les frais de transport pourrait servir les intérêts de la tutelle ne sert pas ceux des structures hospitalières. La T2A inciterait plutôt à faire revenir pour pouvoir facturer. Dans les bases ATIH que nous avons analysées, le ratio entre les actes qui ne sont pas des chimiothérapies et le nombre de séjour, s'il est resté stable entre 2006 et 2009 dans le secteur privé (2,69\%), a baissé sur la même période dans le secteur public (de 19,74\% à 13,80\%). Un établissement de soins en situation précaire du point de vue de l'équilibre financier a tout intérêt à optimiser non pas les séjours des patients, mais son dispositif de facturation. Une consultation auprès d'un cancérologue sera incluse dans la prestation de chimiothérapie si elle est réalisée le même jour, mais sera facturée à part si elle est réalisé un autre jour que la chimiothérapie. La structure même de la tarification met les intérêts de la tutelle en contradiction avec les intérêts des structures hospitalières.

Mais également, étant soumis à une tarification à l'activité unique quels que soient le contenu 
du séjour ambulatoire de chimiothérapie, les gestionnaires hospitaliers doivent pratiquer le maximum de séances de chimiothérapies de toutes durée, et bien sûr si possible simple et courte, pour assurer l'équilibre économique du financement de ce GHM. La dotation attribuée par la T2A concernant les chimiothérapie en séance est un prix forfaitaire, attribué quel que soit les autres acte qui peuvent lui être associés, et quel que soit la durée de l'injection. Les coûts sont lissés sur la totalité des séjours. Les séances qui sont susceptibles d'être réalisées en ambulatoire sont les séances avec les injections les plus courtes et sans soins concomitants. Ce sont donc celles qui tire le coût moyen à la baisse. Un hôpital qui jouerait le jeu d'une externalisation excessive de ses chimiothérapies simples et courtes et ne conserverait que les chimiothérapies longues et/ou associées à d'autres actes, obtiendrait certainement un coût moyen bien supérieur au prix, et donc mettrait en péril son équilibre économique. D’un point de vue comptable, l'administration du traitement anticancéreux à domicile est à même de pénaliser l'institution qui «externalise » les chimiothérapies courtes, c'est à dire celles qui sont le plus intéressantes à faire sortir pour les tutelles de soins.

\section{La chimiothérapie à domicile : une solution avec peu d'avenir ?}

Dans ce contexte, la chimiothérapie à domicile en réseau tend à devenir une solution d'ajustement de l'activité de l'hôpital de jour, sollicitée en cas d'encombrement, ignorée si l'hôpital de jour a besoin d'être rempli. Or, les prescripteurs sont des médecins hospitaliers. Il n'existe donc pas de relation de concurrence, mais plutôt une relation de dépendance. D'autant que les molécules de chimiothérapie, qui se présentent de plus en plus souvent sous forme orale, sont un produit (ou plus exactement une prestation) de substitution qui pourrait limiter le mode d'administration intraveineuse et donc modifier le besoin en structure hospitalière. Or, l'équation économique d'un réseau de soins repose sur une volumétrie suffisante et une régularité des prestations confiées. Ces derniers, qui dépendent de leur partenaires "prescripteur», ne peuvent construire des stratégies optimum sur le plan des dépenses à engager qu'à la condition de pouvoir mutualiser ces dépenses sur plusieurs patients. Ainsi par exemple, trouver des solutions pour coordonner à plus de $100 \mathrm{~km}$ des prises en charge de chimiothérapies à domicile pour des patients en minimisant des coûts de transport suppose de pouvoir négocier avec des pharmacies hospitalières locales, qui n'ont pas forcément de service de cancérologie, mais disposent des compétences pour préparer les molécules, ainsi qu'avec des livreurs locaux (prestataires de service ou taxis), sur des bases qui ne soient pas ponctuelles. 
S'il est clair que la solution d'aller administrer la molécule au domicile du patient lorsque celui-ci habite à quelques pas du centre de cancérologie qui le prend en charge est une solution bien trop coûteuse pour la collectivité, administrer les molécules de chimiothérapies au plus près du lieu de résidence du patient, lorsque ce dernier est éloigné des centres de cancérologie, est malgré tout une solution intéressante sur le plan des coûts d'administration de ces molécules. Les coûts de transport peuvent devenir très importants, le traitement pénible pour le patient. Il existe donc selon nous une place pour des chimiothérapies organisées à proximité du lieu de vie des patients. Si le domicile est une solution résolvant la question de l'éparpillement des malades sur le territoire, des solutions de proximité plus collectives (hôpitaux locaux, centres de soins, maisons pluridisciplinaires) sont à approfondir pour les zones à plus forte densité de population. 


\section{Conclusion}

L'activité de chimiothérapie à domicile ne s'est donc pas vraiment développée, malgré la mesure 41 du plan cancer. La principale raison semble être liée aux modèles d'activité des hôpitaux. La chimiothérapie, qui représente une part importante de l'arsenal thérapeutique de lutte contre le cancer, fait partie du cœur de métier de la cancérologie. Or la cancérologie est une spécialité médicale dont l'exercice est principalement hospitalier. On ne se départit pas facilement de quelque chose qui est autant au cœur de son métier.

Par ailleurs, cette posture est renforcée par les modalités d'allocations de ressources associées à la T2A, qui sont relativement dissuasives pour les hôpitaux. Ces derniers n'ont pas grand intérêt, sauf à avoir un hôpital de jour véritablement saturé, à faire appel à des solutions à domicile pour administrer la chimiothérapie. La solution du domicile devient alors plutôt une variable d'ajustement de la stratégie hospitalière plutôt qu'une solution pérenne, et cela ne permet pas à des structures de réseau stables d'émerger sur cette activité.

La chimiothérapie à domicile, qui est une prestation de soins qui a fait couler beaucoup d'encre, ne s'est développée que faiblement, par absence de promoteurs, probablement (mais sans doute pas seulement) peu stimulée compte tenu de la faiblesse de la demande des hospitaliers pour «sortir» ces prestations de l'hôpital. Les récentes précisions quant à la définition des missions des HAD (loi Hôpital Patient Santé Territoire de 2009) les ont confirmés dans leur mission de construire des alternatives aux hospitalisations classiques et donc infirmés leur positionnement concurrents aux hôpitaux de jour et aux réseaux. Ils ne devraient donc organiser des chimiothérapies à domicile que dans les cas où elles devraient, sans leur participation, faire l'objet d'une hospitalisation de plusieurs jours.

La chimiothérapie à domicile est-elle sur le point de s'éteindre au profit d'une stratégie hospitalière de récupération des activités ? On comprend bien que l'action combinée de la T2A, assortie de l'apparition de molécules administrables par voie orales, délivrées par les pharmacies de ville et pouvant être prises de manière autonome par le patient à son domicile, peut résoudre le problème de l'encombrement des hôpitaux de jour et qu'externaliser ces prestations sur des réseaux de soins ou tout autre solutions extérieurs auraient pour effet de réduire le besoin de place en hôpital de jour.

Pourtant, compte tenu des contraintes liées aux transports des patients, et sous réserve d'une volumétrie permettant de mutualiser un certain nombre de charge, la chimiothérapie à 
domicile coordonnée par un réseau de santé est une solution tout à fait compétitive du point de vue des tutelles sur le plan économique. La condition est qu'elle doit être intégrée dans une gestion globale de la prise en charge, seule à même d'optimiser les parcours. Il faut qu'un modèle se développe sur la base d'une coopération, seule à même de répartir au mieux les patients au regard des contraintes des uns et des autres. La mise en compétition pour l'allocation des ressources, que créé la structure actuelle de la T2A sur cette prestation, n'est pas de nature à favoriser cette coopération. 


\section{Bibliographie :}

INCA (2009), Situation de la chimiothérapie des cancers en 2009, publication disponible sur www.e-cancer.fr.

DGS Direction générale de la Santé (2003) les 70 mesures du Plan Cancer.

HAS Haute Autorités de Santé (2005) Analyse comparée de la chimiothérapie anticancéreuse administrée à l'hôpital ou prise en charge à domicile: aspects économiques et organisationnels,rapport, Juin.

WHYTE W.F. (1989) “Advancing Scientific Knowledge through Participatory Action Research”, Sociological Forum, 4:3, 367-385

WHYTE W.F.(1995) "Encounters with Participatory Action” Qualitive sociology, Fall, vol 18, issue 3, 289-300

ARGYRIS C, PUTMAN R, MACCLAIN SMITH D (1985) “Action Science : Concepts, Methods and Skills for Reasearch and Intervention", San Francisco, Jossey-Bass

OJA, S., \& SMULYAN, L. (1989) Collaborative action research: A developmental approach (Social Research Educational Studies Series, 7). London : Falmer.

HATCHUEL (2008) "Quel horizon pour les sciences de gestion ? Vers une théorie de l'action collective.", in "Les nouvelles foundations des sciences de gestion”, 2ème edition, FNEGE, Vuibert, 2008

BUTHION V, DENECHAUD C, REMONNAY R (2009) «Organisation de la transfusion Sanguine Thérapeutique : Etude des modalités alternatives de la transfusion sanguine thérapeutique organisable en ambulatoire ", Buthion Valérie, Remonnay Raphaël, Denechaud Clémence, Etude réalisé avec le financement de la fondation Alberici-Octalfa, décembre

BAUMARD, P. IBERT J (2003) “Quelles approches avec quelles données”, in "Méthodes de Recherche en Management", Dunod

BOUQUIN H (2008) « Comptablité de gestion », Economica $5^{\text {ème }}$ édition

BUTHION V, DAVID S. (2004) Alternatives à la transfusion sanguine à l'hôpital : pratiques actuelles et perspectives. Gestion Hospitalière; 437 :468-472.

BUTHION V. (2004) Pour une ingénierie de la conception et du fonctionnement des réseaux de soins, propos d'étape d'une analyse de l'externalisation des soins hospitaliers. Revue Gestion 2000 ; 3-4 :53-70.

BUTHION V. (2006) Impact économique d'une cellule de coordination du retour à domicile sur la gestion des flux hospitaliers et l'équilibre financier de l'hôpital. Journal d'Economie Médicale ;2(24) :101-113.

DEGAS Renaud (2009) « Une maison pluridisciplinaire pou reredynamiser l'hôpital local » bulletin du 22 décembre 2009

EDDY D. M.,(2005), « Evidence-Based Medecine : A Unified Approach » Health Affairs, Vol 24 (1) pp 9-17

JUSOT F (2003) « Revenu et Mortalité : Analyse Economique des Inégalités Sociales de Santé en France », Thèse de doctorat en sciences économiques de l'Ecole des Hautes Etudes en Sciences Sociales

COULTER A (1999) “Embracing patient partnership” British Médical Journal, 319:719-94.

CHARLES C., GAFNI A., WHELAN T. (1997) "Shared decision-making in the medical encounter : what does it mean? (or it takes at least two to tango)". Social Science and Medicine. March; 44(5) : 681-92.

CHARLES C., GAFNI A., WHELAN T. (1999) "Decision-making in the physician-patient encounter : revisiting the shared treatment decision-making model". Social Science and Medicine. September ; 49(5) : 651-61.

MOUMJID et al (2009) La prise de décision partagée dans la rencontre médecin-patient : évolution récente et état des lieux dans le cancer du sein en France. Journal d'Economie Médicale. Mai, vol 27, n³:134-145.

MARTINET A.C. (2006) “Stratégie et pensée complexe”, Revue Française de Gestion, №160, pp 31-45

MINTZBERG H (1982) “Structure et dynamique des organisations” Editions des organisations, 434p

LORINO P, (2005) « Contrôle de gestion et mise en intrigue de l'action collective », Revue Française de Gestion, Vol 31, Issue 159, pp 189-209)

WOLCOTT, H. F. (1992) Data Management. In M. C. Alkin (Ed.), Encyclopedia of educational research, Volume 1 (6th ed., pp. 293-299). New York : Macmillan. 
Page 57 sur 122 


\section{Liste des tableaux}

Tableau 1 : Grille de screening des opinions des professionnels de santé sur l'organisation des soins. 18

Tableau 2 : Répartition des séjours en 2009 de la base public. .25

Tableau 3 : dénombrement des séances réalisées pour seul motif de chimiothérapie............................26

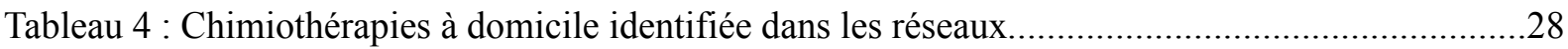

Tableau 5 : récapitulatif des séances de chimiothérapie ambulatoire « simples » réalisées par les

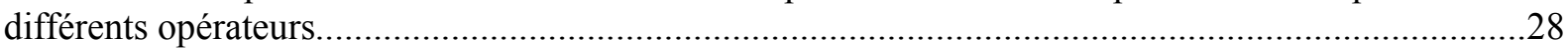

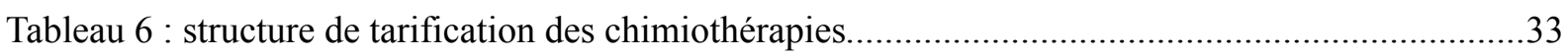

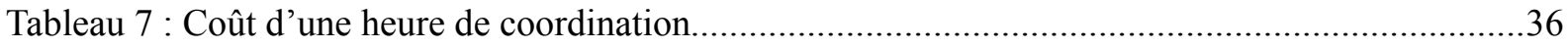

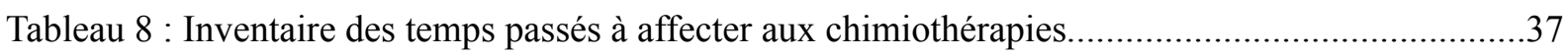

Tableau 9 : Coût complet d'une chimiothérapie réalisée en réseau........................................................37

Tableau 10 : Analyse de l'Echelle Nationale de coûts pour les séjours hospitaliers

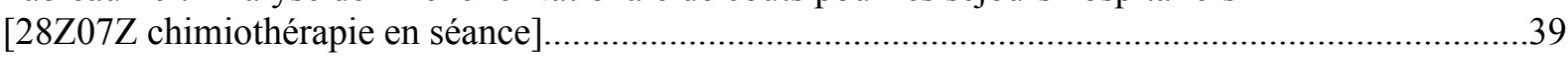

Tableau 11 : Estimation corrigée du coût de la chimiothérapie en Etablissement Hospitalier................40

Tableau 12 : Coût comparé de l'injection d'une chimiothérapie, hors molécule.....................................41

Tableau 13 : Coût de transport des patients, données URCAM..........................................................

\section{Liste des figures}

Figure 1 : Le champ de la diversité des organisations prise en compte dans le cas d'une pathologie

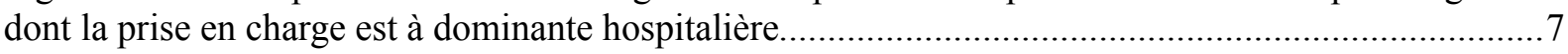

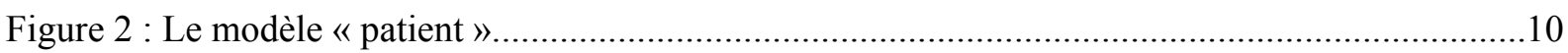

Figure 3 : Déploiement des attributs du modèle "patient" dans une cartographie des espaces de

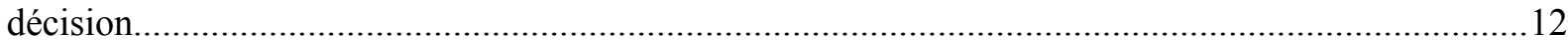

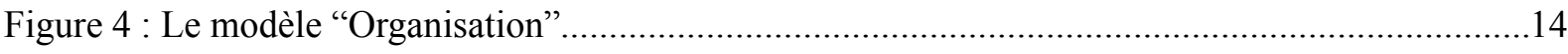

Figure 5 : déploiement des attributs du modèle « organisation » dans une perspective de cartographie

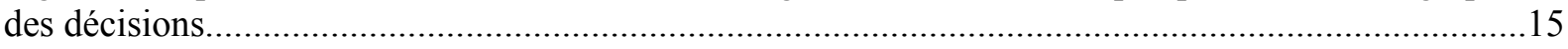

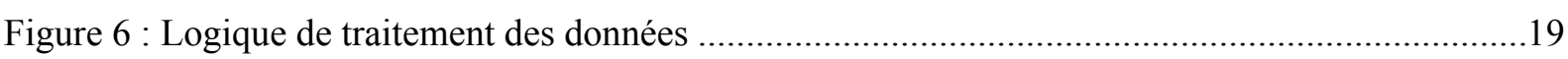

Figure 7 : Simulation du coût pour l'assurance maladie des solutions organisationnelles en fonction de l'éloignement des patients. 
Page 59 sur 122 
ANNEXE 1 :

SYNTHESE DES OPINIONS

ANNEXE 2 :

VOLUMÉTRIE DES SÉANCES DE CHIMIOTHÉRAPIES RÉALISÉES EN AMBULATOIRE.

ANNEXE 3 :

NOTE DE SYNTHÈSE SUR LA QUALITÉ ET LA SÉCURITÉ DES CHIMIOTHÉRAPIES A DOMICILE.

ANNEXE 4 :

RECHERCHE DE LITTÉRATURE SUR LES EFFETS DE SEUIL CRITIQUE DES VOLUMES DE

CHIMIOTHÉRAPIE PRATIQUÉES PAR LES INFIRMIÈRES.

ANNEXE 5 :

ENQUÊTE AUPRÈS DES INFIRMIÈRES LIBERALES SUR LA PRATIQUE DE LA

CHIMIOTHERAPIE A DOMICILE..

ANNEXE 6 :

QUESTIONNAIRE A DESTINATION DES INFIRMIÈRES LIBERALES.

.99

ANNEXE 7 :

COÛT POUR L'ASSURANCE MALADIE DE L'ADMINISTRATION DE LA CHIMIOTHÉRAPIE EN AMBULATOIRE 
Page 61 sur 122 


\section{ANNEXE 1: \\ SYNTHESE DES OPINIONS}

Selon les acteurs des réseaux et des HAD, les patients sont demandeurs de soins à domicile, surtout quand ils demeurent très loin de l'hôpital. Selon les médecins des hôpitaux, les patients préfèrent le côté rassurant de l'hôpital.

Les avis sont assez tranchés entre les partisans des soins à domicile et les partisans de l'activité hospitalière, et il est assez rare de trouver des avis mesurés sur la question.

Pour les patients résidant loin des établissements de soins, les acteurs évoquent la qualité de vie lorsque l'on évite un déplacement. Pour tous, les soignants évoquent l'attente à l'hôpital, et le confort offert aux patients souvent très éprouvés par leur maladie et leur traitement de pouvoir rester chez eux.

Les partisans de l'hôpital évoquent quant à eux la qualité des soins qui y sont dispensés, le côté rassurant pour le patient de pouvoir bénéficier de la présence de l'équipe médicale le cas échéant, et la possibilité, pendant qu'il se trouve à l'hôpital de pouvoir le voir en consultation, de réaliser d'autres soins ou d'autres examens médicaux complémentaires.

L'avis des patients n'est pas collecté de manière fiable. Les opinions des soignants sur ce que pensent les patients est à l'image de ce que pensent les médecins des soins à domicile. Leurs patients sont, selon eux, contents selon eux d'être là où ils sont, quel que soit le lieu.

Il apparaît surtout que, dans une situation où la maladie est particulièrement anxiogène, les patients sont rassurés quand ils se sentent pris en charge sans rupture, par des professionnels coordonnés et sans avoir le sentiment que l'information n'est pas passé entre les acteurs de soins.

Les acteurs des soins à domicile, notamment les infirmières libérales et les promoteurs de réseaux ou certaines HAD, souhaiteraient pratiquer plus de chimiothérapies à domicile, mais les demandes stagnent, voire régressent au fil des années.

La chimiothérapie à domicile est une activité que les acteurs des soins à domicile considèrent comme valorisante. Pour des structures consacrées principalement à des soins dont les hôpitaux se déchargent, et souvent palliatifs, la chimiothérapie donne le sentiment aux soignants d'être capable de coordonner de « vrais » soins. C'est une activité qui a été évoquée comme « noble » par certaines directions d'HAD. 
En 2004, lors de notre première étude sur les soins à domicile, le discours dominant des acteurs des soins à domicile portait sur l'embolisation des hôpitaux de jour et la nécessité de pouvoir décharger les hôpitaux d'une partie de son activité, notamment ambulatoire, afin de permettre de prendre en charge les nouveaux patients qui s'accumulaient à l'entrée. Actuellement, de l'avis des mêmes acteurs, les médecins hospitaliers ne jouent pas le jeu et ne font pas «sortir» les patients. Cette opinion est confirmée par les infirmières libérales formées à la chimiothérapie, qui jugent ne pas pouvoir pratiquer suffisamment faute de patients.

La chimiothérapie à domicile est devenue, après un emballement dans les discours de la fin des années 1990, une pratique rare. Nos interlocuteurs de l'HAD vont même parfois jusqu'à prétendre être quasiment les seuls à en faire encore en France. Beaucoup de projets n'ont pas vu le jour. Par exemple, après avoir installé une équipe pour préparer la mise en œuvre des chimiothérapies à domicile des patients des Hospices Civils de Lyon dans le cadre d'un réseau appelé Onco69, la direction des mêmes hospices à décider fin 2009 d'abandonner ce projet. L'Institut de Cancérologie de la Loire, après avoir obtenu 4 lits d'HAD pour faire de la chimiothérapie à domicile, ne les a jamais utilisés.

La résistance des médecins prescripteurs, la réglementation et le passage à la tarification à l'activité sont les trois raisons invoquées pour expliquer l'absence de véritable demande de la part des hôpitaux. Par ailleurs, lorsque le patient se rend à l'hôpital, il est possible de regrouper visites et soins dans la même journée, et cela permet de mutualiser des frais de transport élevés, mais incontournables pour certaines prestations qui nécessitent les plateaux techniques hospitaliers.

En 2004, la principale cause de résistance invoquée était la crainte des médecins de voir leur patient leur "échapper », plus ou moins masquée par un manque de confiance quant à la sécurité de l'administration à domicile. Cette crainte quant à la qualité des soins persistent chez certains médecins, au point d'utiliser des arguments parfois chocs face au patient pour le dissuader de demander à recevoir sa chimiothérapie à domicile. Une patiente, mère de deux enfants et exaspérée d'attendre pour recevoir ses injections, s'est entendue répondre : «Pas vous, on va vous guérir, on ne va pas vous envoyer vous faire tuer à domicile. » Cela relève d'une méconnaissance de l'organisation mise en place, selon les acteurs de soins à domicile. La chimiothérapie à domicile est aujourd'hui suffisamment encadrée pour que cela ne pose aucun problème de sécurité. 
La réglementation mise en place en 2006 est devenue très contraignante, obligeant la pratique à être encadrée soit pas une HAD, soit par un réseau de soins. Les réseaux de soins se sont très faiblement développés. Certaines zones géographiques ne sont pas couvertes pour les activités de cancérologie. Les HAD n'ont pas vocation a suppléer à l'activité des hôpitaux de jours, puisqu'ils ont pour vocation d'assurer des séjours équivalents aux séjours hospitaliers. Aussi est-ce un peu compliqué pour eux de mettre en place autre chose qu'une activité de dépannage en cas de saturation des hôpitaux de jour, ou pour assurer des chimiothérapies palliatives pour des patients déjà pris en charge par ailleurs.

Actuellement, en plus de ces résistances, le passage à la tarification à l'activité est invoqué comme étant la principale cause de rétention de l'hôpital. Certains établissements ayant augmenté la taille de leur hôpital de jour, il est à présent nécessaire de le remplir pour l'amortir.

Une chimiothérapie peut être d'une durée très variable, allant d'une simple injection à une perfusion de plusieurs heures. Les infirmières libérales ne semblent pas toujours prêtes à rester mobiliser de longues heures au chevet du patient à domicile, les chimiothérapies qui leur sont proposées ne dépassent en général pas 1h30. Dans certaines zones géographique cependant, mobiliser une infirmière libérale pendant plus d'une heure pour un seul patient relève de l'exploit.

Pour certains acteurs hospitaliers, la population des infirmières libérales est considérée comme une ressource disponible et inépuisable. Pour d'autres les infirmières libérales manquent, ou sont fortement saturées par leur activité. La disponibilité des infirmières libérales est très variable selon les zones géographiques.

De plus, certaines infirmières libérales ne sont pas satisfaites de la rémunération de la prestation, notant des contradictions dans la tarification entre une perfusion standard cotée AMI12 et une perfusion de chimiothérapie de moins d'une heure cotée AMI10.

Pourtant, beaucoup de libéraux (médecins, infirmières) apprécient d'être impliqués dans la prise en charge du cancer de patients dont ils se sont occupés dans le passé, et dont ils devront assurer les suites de la prise en charge, après le cancer ou pendant la phase de dégradation et de fin de vie.

Les médecins libéraux se sentent souvent dépossédés lorsque leur patient est pris en charge pour un cancer. Traitement relativement long, le patient peut « disparaitre » dans les couloirs hospitaliers et ne réapparaître que ponctuellement, sans que le généraliste ne connaissent 
véritablement le dossier oncologique, ou au moment où le traitement étant un échec, il faut envisager la prise en charge des derniers mois et la fin de vie. Les généralistes ont alors le sentiment qu'après avoir été ignorés, on leur laisse le «sale boulot». Ceci donne parfois au généraliste un sentiment de rejet vis à vis des patients atteints de cancer, renvoyés pour leur prise en charge à ceux qui possèdent désormais l'essentiel de leur dossier médical, l'hôpital.

Du côté des infirmières libérales, la situation est également différente selon les zones géographiques. Certaines déplorent de ne pas être plus sollicitées pour prendre en charge des chimiothérapies complètes à domicile et trouvent peu valorisant de se voir reléguer à venir débrancher des perfusions posées à l'hôpital. Sur certaines zones géographiques, certaines n'ont jamais cherché à se faire habiliter pour la chimiothérapie, faute de n'avoir jamais été sollicitées pour en réaliser.

Sur le département du Rhône par exemple, beaucoup déplorent de ne pas être plus sollicitées pour prendre en charge des chimiothérapies à domicile. Sur le département de la Loire, il est difficile de trouver une infirmière habilitée, car ces dernières n'ont jamais été sollicitées pour ce genre de prestations.

Dans le Rhône, de nombreuses infirmières ont suivi les formations destinées à leur permettre de prendre en charge les chimiothérapies à domicile. Ces formations sont payantes, et les infirmières trouvent que l'activité n'a pas été au rendez-vous.

C'est l'acte complet d'administration qui est jugé valorisant. La chimiothérapie à domicile est une appellation qui recouvre à la fois cette administration de l'intégralité de la cure, mais également la surveillance ou le débranchement des pompes installées à l'hôpital et avec laquelle le patient rentre à domicile. Les infirmières libérales déplorent que ces demandes soient en croissance alors que celle des actes complets régresse. (cf annexe 2).

Il existe des croyances très ancrées sur le coût de la chimiothérapies à domicile, soit pour dire qu'elle coûte moins cher qu'à l'hôpital, soit pour affirmer le contraire. Les deux composantes évoquées pour faire pencher la balance d'un côté ou de l'autre sont les coûts des transports d'une part, et le temps passé et non mutualisé par les infirmières libérales qui interviennent au domicile des patients, comparés aux hôpitaux de jour.

Les acteurs ont des opinions très tranchées sur les coûts de réalisation de la chimiothérapie. Les tenants des soins à domicile affirment assez facilement que la prestation «coûte » moins cher à domicile, notamment lorsque le patient réside loin de l'hôpital. Le transport en ambulance est évoqué à l'appui de cette affirmation. Les détracteurs affirment le contraire, 
allant parfois jusqu'à dire que les coûts de transport compensent la mobilisation d'une infirmière pour un seul patient. Aucun d'entre eux n'est capable de citer une quelconque étude fiabilisant leurs affirmations.

La chimiothérapie évolue et les laboratoires pharmaceutiques mettent de plus en plus souvent sur le marché des formulations « orales", qui peuvent être administrées par le patient lui-même, sans recours à un personnel soignant. Les impacts de cette formulation sur l'organisation des chimiothérapies et l'effet potentiel sur l'activité des hôpitaux de jour sont actuellement inconnus.

L'administration d'une chimiothérapie orale ne nécessite pas la présence d'un personnel qualifié, le patient pouvant prendre ses cachets de manière autonome. Cette concurrence à l'activité des hôpitaux de jour est pour le moment perçue comme une menace potentielle lointaine.

Certains cancérologues estiment nécessaire d'organiser une supervision spécifique de la chimiothérapie orale pour s'assurer de la compliance du patient. D'autres considèrent que le patient, dont la survie est menacée par le cancer, sera suffisamment concerné pour être autonome. Quoi qu'il en soit, des réflexions se développent sur cet accompagnement. Les HAD en ont été officiellement exclues, les tutelles ayant estimé que cette prestation ne relevait pas de la compétence de l'hospitalisation à domicile. Les réseaux de soins ont été sollicités, mais ils sont peu nombreux et la réflexion est encore embryonnaire.

Enfin, tous les hôpitaux n'ont pas le sentiment d'être saturés, et les investissements récents pour améliorer ou agrandir des hôpitaux de jour rendent l'externalisation de l'activité impossible sur le plan économique.

Si certains hôpitaux ont, ou ont eu, le sentiment d'être saturés par l'activité de chimiothérapie, ce n'est pas le cas de tous. Des investissements d'agrandissement des hôpitaux de jour, des réaménagement ont été réalisés. Or, les hôpitaux de jour en cancérologie ont principalement pour vocation de réaliser des chimiothérapies. Leur externalisation massive pourrait compromettre l'existence même de ces structures. 
Page 67 sur 122 


\section{ANNEXE 2 : \\ VOLUMÉTRIE DES SÉANCES DE CHIMIOTHÉRAPIES RÉALISÉES EN AMBULATOIRE}

L'objectif de cette note est d'identifier quel est le volume réel des séances de chimiothérapies réalisées en ambulatoire, c'est à dire dans un épisode court (sans hébergement) et indépendamment d'autres soins, et quelle est la part des séances réalisées à domicile. Nous allons notamment regarder comment ces chiffres ont évolués compte tenu des mesures relatives au plan Cancer et de l'arrivé de la T2A en 2005. Nous évaluerons le potentiel des séances susceptibles d'être en dehors des plateaux techniques hospitaliers de cancérologie. Les séances de chimiothérapies ambulatoires à l'hôpital

Il existe un GHM unique, le [28Z07Z chimiothérapie pour tumeur, en séances], qui permet de rémunérer la séance, classé dans la CMD des séjours de moins de 24h). Avant 2005, le motif chimiothérapie était inclus dans le GHM 24Z02Z dans les CMD* "séances et séjours de moins 2 jours ».

Dans ces séances, certaines comportent d'autres soins, principalement des radiothérapies, des transfusions sanguines, l'administration d'autres médicaments, et des examens d'imagerie médicale. Nous avons tenté d'en déterminer la proportion afin de dégager le volume des séances ne comportant que l'injection de la molécule anticancéreuse.

\section{La chimiothérapie à l'hôpital}

\subsection{Le dénombrement des séances dans les bases de l'ATIH}

Les données disponibles dans les bases de l'ATIH donne la volumétrie présentée dans le tableau 1.

Tableau 1: Volume des séjours pour chimiothérapie en séance

\begin{tabular}{|l|r|r|r|r|r|}
\cline { 2 - 6 } \multicolumn{1}{c|}{} & \multicolumn{1}{|c|}{2005} & \multicolumn{1}{c|}{2006} & \multicolumn{1}{c|}{2007} & \multicolumn{1}{c|}{2009} \\
\hline $\begin{array}{l}\text { Tous les } \\
\text { établissements }\end{array}$ & 1604608 & 1677711 & 1724267 & 1839772 & 1925656 \\
\hline Public et PSPH & 890157 & 972026 & 1017031 & 1120087 & 1213658 \\
\hline Privé & 714451 & 705685 & 707236 & 719685 & 711998 \\
\hline
\end{tabular}

La figure 1 montre la répartition et met en évidence l'augmentation des séances, $(+20 \%$ en 5 ans), croissance absorbée principalement par le secteur public et PSPH $(+36,34 \%)$, alors que le volume des séances réalisées dans le secteur privé est resté stable).

\footnotetext{
* CMD : Catégorie Majeur de Diagnostic
} 


\section{Figure 1: Répartition et évolution du volume des séances de chimiothérapies réalisées en ambulatoires}

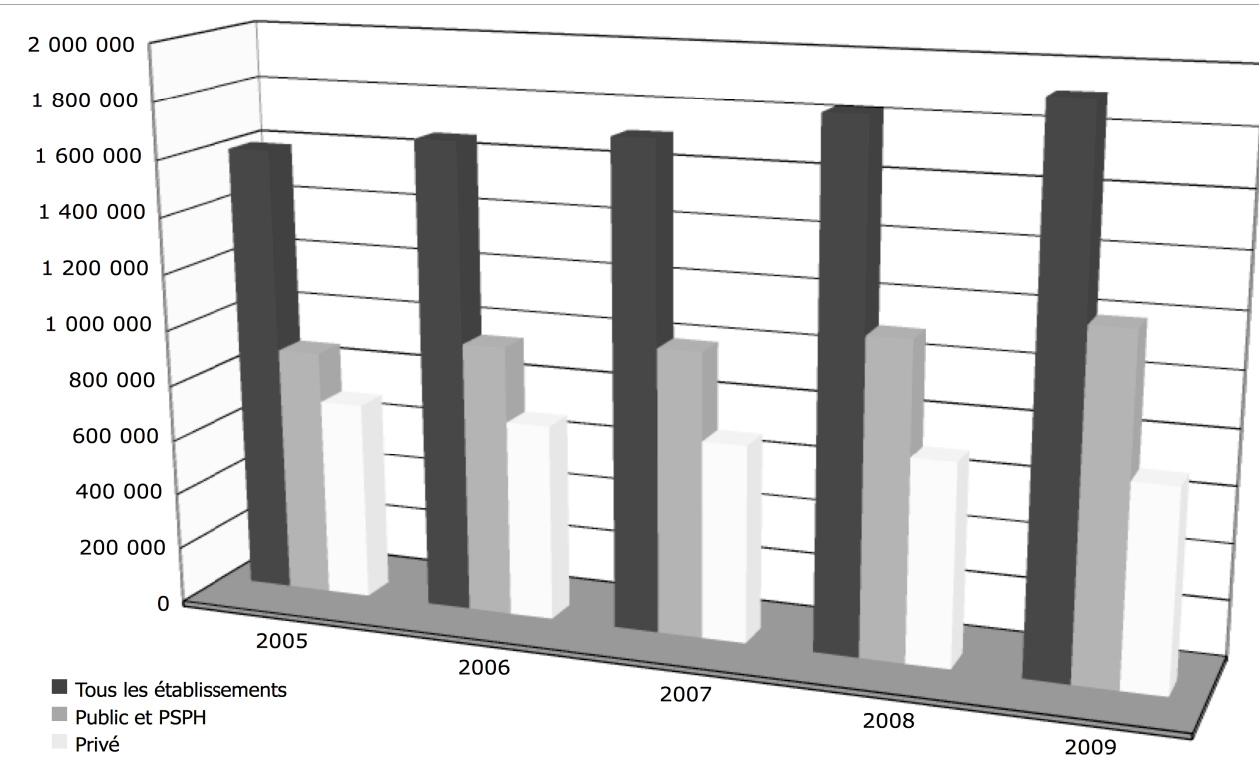

Les hôpitaux public et PSPH s'occupent en moyenne de 60\% des chimiothérapies réalisées en établissement de santé contre $40 \%$ pour le secteur privé. L'évolution progressive de l'incidence des cancers en France ces dernières années (89\% de 1980 et 2005, source INVS, Estimation nationale de l'incidence et de mortalité par cancer en France entre 1980 et 2005) se reflète bien dans les soins qui sont dispensés en chimiothérapie.

Le tableau 2 donne une idée de la répartition en 2009 par type d'hôpitaux de la base publique et PSPH. Ces données confirment que se sont essentiellement les structures techniques les plus grosses qui prennent en charge pour l'essentiel cette prestation. Les gros $\mathrm{CH}$, les $\mathrm{CHR} / \mathrm{U}$ et les CLCC en assurent 85\%.

Tableau 2 : Répartition des séjours en 2009 de la base public.

\begin{tabular}{|l|r|r|}
\hline \multicolumn{1}{|c|}{ Opérateurs } & \multicolumn{2}{|c|}{2009} \\
\hline CH supérieur à 16 000 séjours & 426783 & $35,17 \%$ \\
\hline CHR/U & 368684 & $30,38 \%$ \\
\hline CLCC & 251684 & $20,74 \%$ \\
\hline PSPH & 92579 & $7,63 \%$ \\
\hline CH de 10 001 à 16 000 séjours & 46610 & $3,84 \%$ \\
\hline CH de 5501 à 10 000 séjours & 21940 & $1,81 \%$ \\
\hline CHR de moins de 5500 séjours & 5214 & $0,43 \%$ \\
\hline TOTAL & 1213494 & $100,00 \%$ \\
\hline
\end{tabular}

Nous observons également que les CLCC sont placés en troisième position en ce qui concerne le nombre de séances, pour des raisons de nombre et de taille de ces structures. On dénombre actuellement seulement 20 CLCC contre plus de $450 \mathrm{CH}$ (Fédération Hospitalière Française).

De façon très ponctuelle, certains hôpitaux locaux administrent les chimiothérapies à des patients de leur périmètre géographique, sur demande des hôpitaux de cancérologie et en accord avec les tutelles de financement pour le financement des molécules. Cet accord des 
tutelles est indispensable pour des établissements gérés en budget global, pour qui le financement de molécules souvent très onéreuses mettrait en péril le budget des produits pharmaceutiques. Il n'existe pas de recensement de ces pratiques, nous pouvons considérer qu'elles sont à ce jour très confidentielles.

\subsection{Les séances potentiellement éligibles à une externalisation à domicile}

A l'hôpital, le séjour sera classé dans ce GHM pour toutes les chimiothérapies réalisées en hôpital de jour, quelle que soit leur durée, et compte tenu de l'existence d'autres soins et examens qui peuvent être réalisés concomitamment. Se rendre à l'hôpital peut permettre au patient de bénéficier des soins dans la même journée. Nous avons procédé à une analyse systématique des actes classants et non classants des bases ATIH, en classant les actes relevant de la chimiothérapie elle-mêmes (injections de produits anticancéreux) et les autres. La codification utilisée est présentée dans le tableau 3.

Tableau 3: Classification des actes classants et non classants

\begin{tabular}{|c|c|}
\hline $\mathrm{X}$ & Administration de produits anticancéreux \\
\hline $\mathrm{E}$ & Examen médical (radiologie et autres) \\
\hline $\mathrm{CE}$ & $\begin{array}{c}\text { Complément d'examen médical, actes } \\
\text { accompagnant un examen médical (notamment } \\
\text { administration des agents réactifs pour la } \\
\text { radiologie) }\end{array}$ \\
\hline $\mathrm{R}$ & Radiothérapie \\
\hline $\mathrm{CR}$ & $\begin{array}{c}\text { Complément Radiothérapie, acte } \\
\text { accompagnant une radiothérapie (préparation } \\
\text { des séances) }\end{array}$ \\
\hline $\mathrm{S}$ & Soin \\
\hline $\mathrm{CS}$ & $\begin{array}{c}\text { Complément Soin, actes accompagnant un soin } \\
\text { (anesthésie par exemple) }\end{array}$ \\
\hline $\mathrm{CR}$ & $\begin{array}{c}\text { Complément Radiothérapie, acte } \\
\text { accompagnant une radiothérapie (préparation } \\
\text { des séances) }\end{array}$ \\
\hline $\mathrm{S}$ & Soin \\
\hline $\mathrm{CS}$ & $\begin{array}{c}\text { Complément Soin, actes accompagnant un soin } \\
\text { (anesthésie par exemple) }\end{array}$ \\
\hline
\end{tabular}

Nous avons comptabilisé tous les actes qui n'étaient pas des chimiothérapies. Nous avons associé les actes qui constituent des compléments à d'autre, comme par exemple une anesthésie, qui est concomitante à un soin, une reconstitution tridimensionnelle d'image en radiologie, qui est concomitante d'un examen d'imagerie. Compte tenu de la modification du GHM intervenue à partir de 2006, nous avons mené l'analyse sur les années 2006-2009. Nous ne connaissons pas la répartition moyenne des actes réalisés en plus de la chimiothérapie, et avons donc arbitrairement considéré qu'ils étaient répartis sur différents séjours. Cela nous permet de déterminer a minima le nombre de séance où le patient n'est venu que pour recevoir sa ou ses molécules de chimiothérapie.

Tableau 4 : dénombrement des séances réalisées pour seul motif de chimiothérapie 


\begin{tabular}{|c|c|c|c|c|c|c|c|c|}
\hline & \multicolumn{4}{|c|}{ Public et PSPH } & \multicolumn{4}{|c|}{ Privé } \\
\hline & $\begin{array}{c}\text { Nombre de } \\
\text { séjour dans la } \\
\text { base ATIH }\end{array}$ & $\begin{array}{c}\text { Actes autres } \\
\text { que } \\
\text { chimiothérapie } \\
\text { réalisés dans } \\
\text { les séjours }\end{array}$ & \begin{tabular}{|c|} 
Nombre \\
d'actes \\
rapportés au \\
nombre de \\
séjours
\end{tabular} & $\begin{array}{l}\text { Nombre de } \\
\text { séjour pour } \\
\text { seule chimio }\end{array}$ & \begin{tabular}{|} 
Nombre de \\
séjour dans la \\
base ATIH
\end{tabular} & $\begin{array}{c}\text { Actes autres } \\
\text { que } \\
\text { chimiothérapie } \\
\text { réalisés dans } \\
\text { les séjours }\end{array}$ & $\begin{array}{c}\text { Nombre } \\
\text { d'actes } \\
\text { rapportés au } \\
\text { nombre de } \\
\text { séjours }\end{array}$ & $\begin{array}{l}\text { Nombre de } \\
\text { séjour pour } \\
\text { seule chimio }\end{array}$ \\
\hline 2006 & 972026 & 191890 & $19,74 \%$ & 780136 & 705685 & \begin{tabular}{r|}
18903 \\
\end{tabular} & $2,68 \%$ & 686782 \\
\hline 2007 & 1017031 & 186212 & $18,31 \%$ & 830819 & 707236 & 20480 & $2,90 \%$ & 686756 \\
\hline 2008 & 1120087 & 174056 & $15,54 \%$ & 946031 & 719685 & 19618 & $2,73 \%$ & 700067 \\
\hline 2009 & 1213658 & 167518 & $13,80 \%$ & 1046140 & 711998 & 19176 & $2,69 \%$ & 692822 \\
\hline
\end{tabular}

On constate une présence beaucoup plus forte d'autres actes dans le secteur public et PSPH que dans le secteur privé. Cependant, si le ratio du nombre d'autres actes sur nombre de séjour est resté stable dans le secteur privé, il a décru dans le secteur public. On y voit sans doute les traces d'une préoccupation quant à la gestion des séjours. Rémunérées forfaitairement quels que soient les autres actes réalisés, les structures hospitalières ont intérêt à ne pas regrouper des prestations de soins qui ne modifient pas la rémunération du séjour. Même la consultation de l'oncologue, si elle a lieu le jour de la chimiothérapie, ne peut pas, dans le secteur public et PSPH, être facturée à part. Nous ne sommes cependant pas en mesure d'identifier dans quelle proportion elle a lieu pendant le séjour en hôpital de jour.

Cette estimation permet de déterminer que la grande majorité des séjours (en 2009 86,2\% dans le secteur public et PSPH et 97,31\% dans le privé) ne sont réalisés que pour administrer les molécules. Il n'est pas possible de déterminer quel est la part des molécules qui ne sont pas rétrocédables, ni si l'état de tous les patients autoriserait que l'injection ait lieu ailleurs qu'à l'hôpital. Nous estimerons donc que le chiffre de 1738962 séance en 2009 constitue un maximum pur ce que nous considérons comme « chimiothérapies externalisables ».

\section{Les séances de chimiothérapies à domicile}

Deux acteurs sont identifiés comme pouvant organiser les chimiothérapies à domicile : les HAD et les réseaux de soins. Bien qu'ils puissent apparaître comme concurrent dans la prise en charge, leur rôle est très différents.

\subsection{Les séances de chimiothérapie en HAD}

Les HAD ont été crée pour représenter une alternative aux hospitalisations longues, et non aux hospitalisations de jour. La chimiothérapie étant traditionnellement une activité hospitalière, et les administrations à domicile ne pouvant être effectuées que dans le cadre d'un réseau de soins ou d'une HAD, ces dernières sont intervenues assez naturellement, et se trouve parfois en position de prendre en charge, à la place de l'hôpital de jour, des séances qui auraient été réalisées en ambulatoire. Cette tâche devrait logiquement être dévolue aux réseaux, mais ces derniers étant peu nombreux sur le territoire, les HAD suppléent par défaut. Cependant, La longueur des hospitalisations à domicile pour seul motif de chimiothérapie $(3,8$ jours) peut également laisser penser que ce ne sont pas les mêmes profils de patients que ceux qui sont pris en charge dans les réseaux, ou que les séances dans les hôpitaux de jour pourraient se prolonger pour ces patients.

Les bases de données de l'ATIH donne des éléments rassemblés dans le tableau 5. 
Tableau 5 : description des séjours en HAD comportant des chimiothérapies

\begin{tabular}{|c|c|c|c|c|c|c|c|c|c|c|c|c|}
\hline & \multicolumn{4}{|c|}{\begin{tabular}{|c|} 
Motif Principal \\
$(05$-chimiothérapie)- MA \\
$(00$-aucun)
\end{tabular}} & \multicolumn{4}{|c|}{$\begin{array}{c}\text { Motif Principal } \\
\begin{array}{c}\text { (05-chimiothérapie)- MA } \\
\text { (tous) }\end{array}\end{array}$} & \multicolumn{4}{|c|}{$\begin{array}{l}\text { Motif Principal (tous)-MA (05- } \\
\text { chimiothérapie) }\end{array}$} \\
\hline & $\begin{array}{c}\text { Nombre de } \\
\text { journées }\end{array}$ & \begin{tabular}{|c|} 
Durée \\
moyenne \\
de séjour \\
\end{tabular} & $\mathrm{K}$ & $\begin{array}{l}\text { Nombre de } \\
\text { séquence } \\
\text { ou séjour }\end{array}$ & $\begin{array}{c}\text { Nombre de } \\
\text { journées }\end{array}$ & $\begin{array}{c}\text { Durée } \\
\text { moyenne } \\
\text { de séjour }\end{array}$ & $\mathrm{K}$ & $\begin{array}{c}\begin{array}{c}\text { Nombre } \\
\text { de }\end{array} \\
\text { séquence } \\
\text { ou séjour }\end{array}$ & $\begin{array}{c}\text { Nombre } \\
\text { de } \\
\text { journées }\end{array}$ & \begin{tabular}{|c|} 
Durée \\
moyenne \\
de séjour
\end{tabular} & $\mathrm{K}$ & $\begin{array}{l}\text { Nombre de } \\
\text { séquence } \\
\text { ou séjour }\end{array}$ \\
\hline 2005 & 22091,00 & 3,4 & 70 & 6497 & 50431 & 4,4 & 70 & 11462 & 7404 & 10,4 & 50 & 712 \\
\hline 2006 & 27056,00 & 2,9 & 70 & 9330 & 65943 & 3,9 & 70 & 16908 & 13077 & 15 & 50 & 872 \\
\hline 2007 & 24359,00 & 2,9 & 70 & 8400 & 69102 & 3,9 & 70 & 17718 & 16612 & 11,6 & 50 & 1432 \\
\hline 2008 & 26979,00 & 3 & 70 & 8993 & & 4 & 70 & 18305 & 20035 & 14,5 & 50 & 1382 \\
\hline 2009 & 47206,00 & 3,8 & 70 & 12423 & 79943 & 4,3 & 70 & 18591 & 23653 & 17,3 & 50 & 1367 \\
\hline
\end{tabular}

La chimiothérapie peut être réalisée à titre de prise en charge principale, associée ou non à d'autres soins, ou à titre de prise en charge associée, avec d'autres motifs de prise en charge principale. $\mathrm{K}$ indique l'indice de Karnosky, qui mesure l'état de dépendance de la personne. Un indice de 70 indique une personne qui peut assurer ses soins, mais incapable d'exécuter une activité normale ou de faire un travail actif. Un indice de 50 indique une personne dans une dépendance totale, exige une assistance considérable et un soin médical fréquent. On trouvera dans cette catégorie des patients dans un état de santé dégradé, qui sont entrés dans une logique de soins plus conséquents et bénéficient de chimiothérapie à visée plus palliative.

Les patients susceptibles de recevoir leurs chimiothérapies en hôpital de jour, et de ne s'y rendre que pour cela, sont les patient inclus en HAD pour seul motif de chimiothérapie. Malgré un doublement du nombre de séjours (de 6497 à 12422) sur les cinq années étudiées, la chimiothérapie à domicile pratiquée par les HAD est très faible. Le graphique ci-dessous montre bien le poids important des chimiothérapies exercées en ambulatoire, celles à domicile restant très faibles par rapport au 1,7 millions identifiées comme potentiellement externalisables dans le paragraphe précédent.

\subsection{Les séances de chimiothérapie en réseau}

Le second intervenant potentiel pour la chimiothérapie à domicile est le réseau territorial de cancérologie. Il n'existe pas de bases statistiques centralisées, aussi est-il difficile de connaître les volumes de chimiothérapies qui y sont réalisées.

En 2005, un rapport de la Haute Autorité de Santé faisait état de 4 réseaux pratiquant la chimiothérapie à domicile. Nous avons cherché les informations sur internet, "réseaux territoriaux de cancérologie ou conologie», puis contacté ceux qui faisait état d'expérience de chimiothérapie à domicile. En sus du réseau Source à Lyon, nous en avons contacté 5 (Réseau territorial de Nantes, Réseau Oncorient, Réseau Onco Pays de Loire, Réseau Onco Loire Estuaire, Réseau Oncolor et réseau Onconord). Oncolor et Onconord nous a répondu qu'ils ne pratiquaient pas la chimiothérapie à domicile. Nantes et Oncorient ne nous ont pas répondu. Les informations que nous avons pu collecter sont rassemblées dans le tableau 6.

Tableau 6: Chimiothérapie à domicile identifiée dans les réseaux

\begin{tabular}{|l|r|r|}
\cline { 2 - 3 } \multicolumn{1}{c|}{} & $\mathbf{2 0 0 8}$ & $\mathbf{2 0 0 9}$ \\
\hline Source (Lyon) & 35 & 532 \\
\hline OncoLoire (Saint & & \\
Nazaire, St Herlin)) & 357 & 464 \\
\hline Onco Loire Estuaire & & 1229 \\
\hline TOTAL & $\mathbf{3 9 2}$ & $\mathbf{2 2 2 5}$ \\
\hline
\end{tabular}


Cet inventaire est probablement incomplet, mais le informations manquantes sont probablement assez faible, comme le sont les chiffres présentés ici. La pratique de la chimiothérapie via les réseaux de soins est très faiblement développée.

\section{Conclusion : La faiblesse de la chimiothérapie ambulatoire réalisée en dehors de l'hôpital}

Nous récapitulons dans le tableau 7 le dénombrement des séances de chimiothérapie « simples », c'est-à-dire non accompagnées d'autres soins réalisés de façon concomitante.

Tableau 7 : récapitulatif des séances de chimiothérapie ambulatoire « simples » réalisées par les différents opérateurs

\begin{tabular}{|l|r|r|r|r|r|r|r|r|}
\hline & \multicolumn{2}{|c|}{2006} & \multicolumn{2}{c|}{2007} & \multicolumn{2}{c|}{2008} & & \\
\hline $\begin{array}{l}\text { Hôpitaux } \\
\text { Publics et } \\
\text { PSPH }\end{array}$ & 780136 & $52,8 \%$ & 830819 & $54,4 \%$ & 946031 & $57,1 \%$ & 1046140 & $59,7 \%$ \\
\hline $\begin{array}{l}\text { Hôpitaux } \\
\text { privés }\end{array}$ & 686782 & $46,5 \%$ & 686756 & $45,0 \%$ & 700067 & $42,3 \%$ & 692822 & $39,5 \%$ \\
\hline HAD & 9330 & $0,6 \%$ & 8400 & $0,6 \%$ & 8993 & $0,5 \%$ & 12423 & $0,7 \%$ \\
\hline $\begin{array}{l}\text { Réseaux de } \\
\text { cancérologie }\end{array}$ & & & & & 392 & $0,0 \%$ & 2225 & $0,1 \%$ \\
\hline TOTAL & 1476248 & $100 \%$ & 1525975 & $100 \%$ & 1655483 & $100 \%$ & 1753610 & $100 \%$ \\
\hline
\end{tabular}

La chimiothérapie à domicile représentait en 2009 moins de $1 \%$ des prises en charge. Les réseaux ont pour le moment une place insignifiante. Leur émergence tardive dans le paysage sanitaire peut expliquer leur faible place, mais ce qui se passe dans les lieux où ils sont présents tendrait à montrer que leur existence ne suffit à provoquer une externalisation importante. Pour le réseau Source, intervenant sur une bonne partie du département du Rhône, le nombre de séance réalisées en 2009 représente $0,81 \%$ de l'activité de chimiothérapie ambulatoire du département (65 026 séances enregistrées dans la bases de l'ATIH sur le Rhône, tous établissements confondus). Pour son principal prescripteur, le Centre Léon Bérard, cela a représenté $2,89 \%$ de l'activité prescrite (17 866 séjours déclarés en 2009). Certes, la jeunesse du réseau nécessite une montée en charge progressive, mais la demande début 2010 reste en relativement faible croissance. L'abandon par les Hospices Civils de Lyon de leur projet de réseau pour la chimiothérapie à domicile, va dans le même sens.

L'activité de chimiothérapie à domicile ne s'est donc pas vraiment développée, malgré la mesure 41 du plan cancer. La principale raison semble être liée aux modèles d'activité des hôpitaux, qui absorbe la montée- en charge. Par ailleurs, cette posture est renforcée par les modalités d'allocations de ressources associées à la T2A, qui sont relativement dissuasives pour les hôpitaux. Ces derniers n'ont pas grand intérêt, sauf à avoir un hôpital de jour véritablement saturé, à faire appel à des solutions à domicile pour administrer les chimiothérapies les plus courtes. La solution du domicile devient alors plutôt une variable d'ajustement de la stratégie hospitalière plutôt qu'une solution pérenne, et cela ne permet pas à des structures de réseau stables d'émerger sur cette activité. 


\section{ANNEXE 3 : \\ NOTE DE SYNTHÈSE SUR LA QUALITÉ ET LA SÉCURITÉ DES CHIMIOTHÉRAPIES A DOMICILE}

Nous avons souhaité vérifier dans la littérature scientifique comment les prises en charges des chimiothérapies anticancéreuses à domicile et en hospitalisation conventionnelle avaient été comparées et quels en avaient été les résultats. Nous voulons ainsi exclure l'hypothèse que le moindre développement des solutions à domicile pourraient être lié à une crainte quant à la moindre efficacité des soins qui aurait été identifiée dans la littérature.

\section{Stratégie de recherche}

La recherche de la littérature scientifique a été effectuée dans différentes bases de données. La période étudiée est de 1980 à nos jours. Nous avons utilisé des Bases de données bibliographiques automatisées comme MEDLINE (National library of medicine, États-Unis), PASCAL (CNRS-INIST, France), WEB OF SCIENCE (ISI Web of knowledge). Les autres sources sont: BDSP (Banque de données en santé publique, Rennes), Internet avec des moteurs de recherche (google...).

Les termes de recherche sont soit des termes issus d'un thesaurus (descripteurs du MESH pour MEDLINE), soit des termes du titre ou du résumé (mots libres). Ils sont combinés en autant d'étapes que nécessaire à l'aide des opérateurs « ET » « OU » «SAUF ». La stratégie de recherche est détaillée dans le tableau 1.

Tableau1: Stratégie de recherche bibliographique

\begin{tabular}{|c|c|c|c|}
\hline Sujet & Termes Utilisés & Période & $\begin{array}{l}\text { Nombre de } \\
\text { références }\end{array}$ \\
\hline Recommandation & $\begin{array}{l}\# 1: \text { « Home » or « outpatient } » \text { or } \\
\text { « home care » or « domiciliary } \\
\text { chemotherapy» } \\
\text { AND } \\
\# 2 \text { « chemotherapy* » or } \\
\text { « intravenous chemotherapy » } \\
\text { AND } \\
\# 3 \text { « cancer*» }\end{array}$ & $\begin{array}{c}1980 \text { à } \\
\text { aujourd'hui }\end{array}$ & 496 \\
\hline feasability & $\begin{array}{l}1 \\
\text { AND } \\
\# 2 \\
\text { AND } \\
\# 3 \\
\text { AND } \\
\# 4 \text { « quality » or « security » }\end{array}$ & $\begin{array}{c}1980 \text { à } \\
\text { aujourd'hui }\end{array}$ & 142 \\
\hline Complications & $\begin{array}{l}1 \\
\text { AND } \\
\# 2 \\
\text { AND } \\
\# 3 \\
\text { AND } \\
\# 5 \text { « side effects » or « adverses } \\
\text { effects» }\end{array}$ & $\begin{array}{c}1980 \text { à } \\
\text { aujourd'hui }\end{array}$ & 211 \\
\hline
\end{tabular}


Le nombre total de référence obtenues est de 849 . Le nombre d'articles pertinents retenus est de 11 .

\section{Comparaison des études}

Les études prises en compte sont inventoriées dans le tableau 2.

Tableau 2: descriptif des articles retenus

\begin{tabular}{|c|c|c|c|}
\hline $\begin{array}{l}\text { Référen } \\
\text { ce }\end{array}$ & Traitement & Méthodologie & Résultats \\
\hline $\begin{array}{l}\text { Vokes } \\
\qquad \begin{array}{l}\text { \& } \\
\text { al.,1 } \\
989\end{array}\end{array}$ & $\begin{array}{l}\text { Cancer tête et cou. } \\
\text { Même traitement } \\
\text { dans les } 2 \text { groupes } \\
(5-F U \text { IV) } \\
\mathrm{N}=19\end{array}$ & $\begin{array}{l}\text { Essai randomisé } \\
\text { croisé. }\end{array}$ & $\begin{array}{l}1 \text { décès au domicile, aucune preuve } \\
\text { significative ne met en cause le domicile. } \\
\text { Pas de défaillance technique, pas de } \\
\text { différence significative dans la fréquence } \\
\text { ou la gravité des effets indésirables } \\
\text { domicile vs hôpital. }\end{array}$ \\
\hline $\begin{array}{l}\text { Rishin } \\
\& \text { al. } \\
2000\end{array}$ & $\begin{array}{l}\text { Cancer (non } \\
\text { précisé) traitement } \\
\text { IV N=20 }\end{array}$ & $\begin{array}{l}\text { Essai randomisé } \\
\text { croisé. }\end{array}$ & $\begin{array}{l}\text { Aucune complication majeure de la } \\
\text { chimiothérapie à domicile n'a été } \\
\text { répertoriée. } \\
\text { Les patients préfèrent la chimiothérapie à } \\
\text { domicile à un traitement en milieu } \\
\text { hospitalier. }\end{array}$ \\
\hline $\begin{array}{l}\text { Borras } \\
\& \text { al. } \\
2001\end{array}$ & $\begin{array}{l}\text { Cancer colorectal } \\
\text { Même traitement } \\
\text { dans les } 2 \text { groupes } \\
(5-\mathrm{FU} \text { IV }) \\
\mathrm{N}=45 \text { vs } \mathrm{N}=42 \mathrm{a}\end{array}$ & $\begin{array}{l}\text { Essai contrôlé } \\
\text { randomisé. }\end{array}$ & $\begin{array}{l}\text { La toxicité des traitements anticancéreux } \\
\text { était similaire dans les } 2 \text { groupes. } \\
\text { La chimiothérapie à domicile semble être } \\
\text { une alternative acceptable et sécuritaire } \\
\text { pour les traitements ambulatoires et } \\
\text { pourrait améliorer la satisfaction et } \\
\text { l'observance du traitement. }\end{array}$ \\
\hline $\begin{array}{l}\text { Lowent } \\
\text { hal } \\
\text { \& al. } \\
1996\end{array}$ & $\begin{array}{l}\text { Différents cancers. } \\
\text { Même ttt ds les } 2 \\
\text { grpes. } \\
\mathrm{N}=65 \text { (domicile) vs } \\
\mathrm{N}=119 \text { (hôpital) }\end{array}$ & Etude contrôlé & $\begin{array}{l}\text { La plupart des complications restent } \\
\text { mineurs à l'hôpital comme au domicile. } \\
\text { Le traitement à domicile a été bien } \\
\text { accueilli par les patients atteints de } \\
\text { cancer et s'est avéré être tout à fait sûr. }\end{array}$ \\
\hline $\begin{array}{l}\text { Brown } \\
\& \text { al. } \\
1997\end{array}$ & $\begin{array}{l}\text { Différents cancers. } \\
\text { Même ttt ds les } 2 \\
\text { grpes. } \\
\mathrm{N}=152 \text { (32 } \\
\text { exclusivement à } \\
\text { domicile et120 à } \\
\text { domicile et hôpital } \\
\text { ou seulement à } \\
\text { l'hôpital) }\end{array}$ & $\begin{array}{l}\text { Etude } \\
\text { rétrospective }\end{array}$ & $\begin{array}{l}\text { Aucune différence dans les } \\
\text { complications pour les patients recevant } \\
\text { une administration de la chimiothérapie à } \\
\text { domicile vs milieu hospitalier n'a été } \\
\text { relevée. }\end{array}$ \\
\hline $\begin{array}{l}\text { Malone } \\
\text { \& al. }\end{array}$ & $\begin{array}{l}\text { Cancer } \\
\text { gynécologique }\end{array}$ & Essai contrôlé & $\begin{array}{l}\text { Absence d'incidents grave de type } \\
\text { réaction anaphylactique ou }\end{array}$ \\
\hline
\end{tabular}




\begin{tabular}{|c|c|c|c|}
\hline 1986 & $\begin{array}{l}\text { Polychimiothérapi } \\
\text { e avec de la } \\
\text { cisplatine, ttt à } \\
\text { domicile après une } \\
\text { première cure à } \\
\text { l'hôpital. } \\
\mathrm{N}=15\end{array}$ & & $\begin{array}{l}\text { extravasation. } \\
\text { Pas de ré hospitalisation liés aux effets } \\
\text { secondaires. }\end{array}$ \\
\hline $\begin{array}{l}\text { DeMoos } \\
\& \text { al. } \\
1980\end{array}$ & $\begin{array}{l}\text { Polychimiothérapi } \\
\text { e } \\
\text { ttt à domicile (IV) } \\
\mathrm{N}=70\end{array}$ & $\begin{array}{l}\text { Etude } \\
\text { rétrospective }\end{array}$ & $\begin{array}{l}\text { trop médical et pas lié au domicile, } \\
\text { mettre ici les conclusions de l'étude sur } \\
\text { le domicile }\end{array}$ \\
\hline $\begin{array}{l}\text { Virally } \\
\& \text { al. } \\
2003\end{array}$ & $\begin{array}{l}\text { CBNPC } \\
\text { Vinorébline (IV) + } \\
\text { gemcitabine (IV). } \\
\mathrm{N}=74(401 \\
\text { injections à } \\
\text { domicile) }\end{array}$ & $\begin{array}{l}\text { Etude } \\
\text { rétrospective (sur } \\
\text { 5ans) }\end{array}$ & $\begin{array}{l}\text { Présence de quelques } \\
\text { dysfonctionnements et incidents mais } \\
\text { insuffisants pour empêcher la réalisation } \\
\text { de la chimiothérapie prescrite. Toxicités } \\
\text { sont mineures et gérées au domicile. } \\
\text { La chimiothérapie à domicile du cancer } \\
\text { bronchique par Vinorébline ou } \\
\text { Gemcitabine est réalisable mais nécessite } \\
\text { une organisation spécifique pour en } \\
\text { assurer la sécurité. }\end{array}$ \\
\hline $\begin{array}{l}\text { Anderso } \\
\text { n \& al. } \\
2003\end{array}$ & $\begin{array}{l}\text { CBNPC non } \\
\text { opérables } \\
\text { Gemcitabine (IV) } \\
\mathrm{N}=24(249 \\
\text { injections à } \\
\text { domicile) }\end{array}$ & $\begin{array}{l}\text { Etude contrôlé } \\
\text { Le premier cycle } \\
\text { du traitement } \\
\text { anticancéreux } \\
\text { s'est fait à } \\
\text { l'hôpital }\end{array}$ & $\begin{array}{l}\text { Déces : AVC ( } \mathrm{n}=1) \text { et cancer du poumon } \\
\text { ( } \mathrm{n}=6) \text { mais aucune donnée ne relie ces } \\
\text { décès au soins à domicile. L'ensemble } \\
\text { des réactions toxiques est dans } \\
\text { l'ensemble de gravité moyenne (grade I } \\
\text { et II, échelle OMS). Aucune toxicité de } \\
\text { grade 4. Cette étude de faisabilité } \\
\text { indique que le transfert de } \\
\text { l'administration d'un agent unique } \\
\text { comme la Gemcitabine à domicile, avec } \\
\text { soins infirmiers efficaces, soutien interne } \\
\text { et communication claire) est acceptable } \\
\text { pour les patients et les soignants. }\end{array}$ \\
\hline $\begin{array}{l}\text { Réseau } \\
\text { OCLE } \\
2007\end{array}$ & $\begin{array}{l}\text { Différents cancers } \\
\text { (non précisé). } \\
\text { Polychimiothérapi } \\
\text { e (IV à domicile) } \\
\mathrm{N}=499\end{array}$ & $\begin{array}{l}\text { Etude } \\
\text { rétrospective sur } \\
3 \text { ans (non } \\
\text { contrôlé). }\end{array}$ & Aucun incident notable relevé. \\
\hline $\begin{array}{l}\text { Lewden } \\
- \\
\text { Bernada } \\
\text { c \& al. } \\
2008\end{array}$ & $\begin{array}{l}\text { Cancers (sein, } \\
\text { ORL, pancréas, } \\
\text { côlon, ovaire et } \\
\text { vessie). } \\
\text { Polychimiothérapi } \\
\text { e (IV et à }\end{array}$ & Essai contrôlé & $\begin{array}{l}\text { Aucun incident majeur n'a été détecté. } \\
\text { Cette expérimentation valide la } \\
\text { faisabilité d'une prise en charge à } \\
\text { domicile à des patients atteints de cancer } \\
\text { dont la typologie a été validée par le } \\
\text { réseau Onco Pays-de-la-Loire, ce en }\end{array}$ \\
\hline
\end{tabular}




\begin{tabular}{|l|l|l|}
\hline domicile) & $\begin{array}{l}\text { s'appuyant sur une coordination } \\
\text { infirmière et pharmaceutique. } \\
\text { Effets secondaires ou indésirables non } \\
\text { précisés dans l'étude }\end{array}$ \\
\hline
\end{tabular}

Les articles trouvés ont été écrits dans les années 90 et plus tard. Cette représentation est dû au fait que la pertinence de cette question est récente.

Un essai randomisé datant de 1989 [Vokes \& al., 1989] mené sur 19 patients traités par 5-FU³ (IV) pour des cancers de la tête et du cou soit à domicile (avec un appareil Infusor), soit à l'hôpital (avec une pompe standard ou Infusor). Les traitements étaient appliqués en plan croisé (domicile ou hôpital) avec chacun une période de cinq jours. Certains patients ont eu des effets indésirables des traitements anticancéreux avec une différence de symptomatologie domicile vs hôpital. A l'hôpital on retrouve une infection locale, une tubulure bouchée, une thrombose veineuse profonde et une thrombocytopénie liées au dispositif d'accès veineux, chez cinq patients. A domicile les effets sont plus d'ordre toxiques (fièvre, inflammation de la muqueuse, aplasie médullaire). La différence marquante dans cette étude est un décès survenu à domicile lié à une défaillance cardiaque qui reste un facteur de risque important des cancers de la tête et du cou. Notant qu'il n'y avait aucune différence significative sur les 19 patients, dans la fréquence ou la gravité des effets indésirables entre le traitement à domicile et à l'hôpital, l'étude semble nous indiquer implicitement que la mort à domicile aurait pu aussi bien se déclarer à l'hôpital. On ne note aucune défaillance technique ni de différence significative dans la fréquence ou la gravité des effets indésirables à domicile vs hôpital.

L'étude relevée dans le Medical Journal Australian [Rishin \& al. , 2000] au sujet d'un essai randomisé croisé comportait 20 patients qui ont été assignés au hasard pour recevoir leur traitement de chimiothérapie de cycle 1 soit à la maison ou l'hôpital, et le second cycle était permuté. Dans cette étude, les effets secondaires ou indésirables de la chimiothérapie anticancéreuse ne figurent pas. Il nous est juste indiqué qu'aucune complication majeure de la chimiothérapie à domicile n'a été reportée. Aucune donnée sur d'éventuels décès n'est notée.

Borras et ses collègues [2001] ont mené un essai randomisé contrôlé sur deux groupes adultes atteint d'un cancer colorectal et traités par 5-FU (IV), domicile $(n=45)$ vs ambulatoire $(n=42)$. Les sorties prématurées de l'étude pour raisons médicales (toxicités, progression de la maladie) étaient plutôt similaire dans les deux groupes : $24 \%$ (11 patients) à domicile et $30 \%$ (13 patients) en ambulatoire. Pour d'autres raisons que médicale, on dénombre $35 \%$ de patients sur la population total (87) ayant arrêté l'essai avec une différence observée entre les patients traités à domicile $(26,5 \%)$ et ceux traités en ambulatoire $(45 \%)$. La toxicité des traitements anticancéreux était similaire dans les 2 groupes. Aucune donnée sur d'éventuels décès n'est observée.

Lowenthal et ses collègues [1996] ont dirigé une étude rétrospective visant à traiter deux populations atteints de cancer de localisation variées, par chimiothérapie anticancéreuse*; une à domicile avec 65 patients (englobant le lieu de travail, le cabinet du médecin généraliste) et l'autre à l'hôpital avec 119 patients. Une réaction au traitement anticancéreux nommée dystonie est à noter à domicile et à nécessiter un traitement à l'hôpital. La plupart des complications restent mineures à l'hôpital comme au domicile. Le traitement à

\footnotetext{
3 5-fluoro-uracile, traitement anticancéreux appartenant à la classe des antimétabolites.
} 
domicile a été bien accueilli par les patients atteints de cancer et s'est avéré être tout à fait sûr.

L'étude rétrospective conduit par Brown et ses collègues en 1997 [Brown \& al., 1997] voulait identifier la fréquence des complications chez les patients recevant une chimiothérapie par dispositifs d'accès veineux implantables. Cette étude permettait également de préciser si des complications étaient associées au mode de prestation de chimiothérapie (schémas bolus ou en perfusion), et d'évaluer l'influence des autres facteurs de risque incluant l'administration des traitements à domicile versus hôpital. L'âge des patients, la fréquence d'accès à la chambre implantable, le type de tumeur, et la neutropénie ont été évalués comme facteurs de risque, mais aucun n'a été statistiquement significatif. On note 3 patients (sur les 32 traités exclusivement à domicile) ayant eu une ablation de la voie d'accès. D'après l'étude, $24 \%$ des patients ont eut des complications à l'hôpital et/ou à l'extérieur de l'hôpital. Ce manque de précision du nombre précis de patients traités soit au domicile soit à l'hôpital engendre certaines difficultés dans la comparaison domicile versus hôpital. Les complications liées au dispositif d'accès veineux implantables ont été peu fréquentes. Aucune différence dans les complications pour les patients recevant une administration de la chimiothérapie à domicile vs milieu hospitalier n'a été relevée.

Une analyse datant de plus de 20 ans fut pilotée au Etats-Unis [De Moos, 1980]. Le rapport de cette étude montre qu'une moyenne de 400 traitements anticancéreux a été administrée à domicile, à plus de 70 patients âgés de 29 à 90 ans et présentant différents cancers. Les effets secondaires remarqués à domicile sont minimes : des saignements légers sans gravité en rapport à des thrombocytopénie, légers saignements rectal $(\mathrm{n}=2)$, des vomissements et une hyperpigmentation d'origine veineuse. Un arrêt du traitement était en lien avec une grave inflammation de la muqueuse. D'après cette étude, plusieurs patients ont subi au domicile, une aplasie médullaire à plusieurs degrés. Celle-ci est considérée comme une conséquence de la toxicité du traitement, et aurait donc pu se dérouler en milieu hospitalier. Il n'est pas précisé d'incidents graves ou de décès au domicile.

Dans le cadre d'une étude française portant sur des patients atteints de cancer bronchique non à petites cellules (CBNPC), 74 patients ont été traités à domicile soit 401 injections $(72 \%$ vinorébline et $28 \%$ Gemcitabine) [Virally \& al. , 2003]. L'ensemble des toxicités étaient mineures : vomissement $(n=1)$, fièvre et syndrome grippal $(n=5)$, extravasation avec nécrose cutanée localisée $(n=2)$, et ont été gérées au domicile. L'essai indique la présence de quelques dysfonctionnements et incidents mais insuffisants pour empêcher la réalisation de la chimiothérapie prescrite. Les toxicités sont mineures et gérées au domicile. La chimiothérapie à domicile du cancer bronchique par Vinorébline ou Gemcitabine est réalisable mais nécessite une organisation spécifique pour en assurer la sécurité.

Dans le cadre d'une étude anglaise portant sur des patients atteints de cancer du poumon non à petites cellules (CBNPC) et non opérables, 24 patients ont reçu un traitement anticancéreux nommé Gemcitabine sur une durée de 14 mois [Anderson \& al ; , 2003]. Le but était d'évaluer la faisabilité et l'acceptabilité de l'administration de la gemcitabine en monothérapie chez les patients atteint du CBNPC, à domicile. Un total de 249 injections de gemcitabine a été réparti en six cycles. Tous les patients ont reçu leur premier cycle de chimiothérapie à l'hôpital (72 perfusions). En tout, 20 patients (sur 24) ont arrêté la chimiothérapie, cinq d'entres eux étaient dus à des vomissements (cycle 3), de l'hypertension (cycle 2), symptômes pseudo-grippaux (cycle 1) et dyspnée (cycle 2 et 4). Un patient est décédé d'un accident vasculaire cérébral et six de cancer du poumon, mais aucune donnée ne stipule que ces décès sont dus aux soins à domicile. L'ensemble des réactions toxiques est dans l'ensemble de gravité moyenne (grade I et II, échelle OMS). Aucune toxicité de grade 4 n'est constatée. Cette étude de 
faisabilité indique que le transfert de l'administration d'un agent unique au domicile comme la Gemcitabine (avec soins infirmiers efficaces, soutient interne et communication claire) est acceptable pour les patients et les soignants.

Une autre observation prospective a été réalisée par le réseau territorial de Saint Nazaire [2007] pour une période de trois ans d'activité de chimiothérapie à domicile. Un total de 499 patients a été pris en charge. Plusieurs classes de chimiothérapie ont été utilisées avec des traitements antimitotiques intraveineux ${ }^{4}$ et des biphosphanates (Zometa/Aredia). Pendant le suivi, 12 arrêts à domicile ont été dénombrés soit à la demande du patient, soit à la demande de l'oncologue pour des motifs de tolérance au traitement ou de difficultés de perfusion sur site implantable. Aucun incident notable n'est relevé.

Lewden-Bernadac et ses collègues [2008] ont mené une étude expérimentale sur 41 patients adultes pris en charge sur le site nord du Réseau Territorial de Nantes, domiciliés sur le département de Loire-Atlantique et ayant donné leur accord pour une administration d'une chimiothérapie injectable ${ }^{5}$ à domicile. Depuis le début de l'essai, il y a eu 21 arrêts de traitement (11 pour amélioration de l'état, 4 suite à l'évolution de la maladie et 6 décès). Les effets secondaires ou indésirables ne sont pas présents dans l'expérience. Aucun incident majeur n'a été détecté. Cette expérimentation valide la faisabilité d'une prise en charge à domicile à des patients atteints de cancer dont la typologie a été validée par le réseau Onco Pays-de-la-Loire, ce en s'appuyant sur une coordination infirmière et pharmaceutique.

\section{Discussion}

La cancérologie ne permet pas d'effectuer des essais randomisés en double aveugle. Il ne serait pas moral d'administrer un traitement où, ni le patient, ni le médecin, ne connaît la molécule. Dans les cas où l'on étudie le lieu de traitement, et non la molécule, le patient et le médecin ne peuvent a fortiori pas ignorer le lieu dans lequel ils se trouvent. Dès lors, les études étant ouvertes, le poids d'efficacité de celles-ci peut être remis en cause sur le plan de la norme méthodologique standard.

Toutefois, la chimiothérapie à domicile semble réalisable au regard des différentes études analysées. En effet, aucune ne relate un ou plusieurs incidents majeurs reliés strictement au domicile. D'après l'étude de Vokes et ses collègues [1989], on observe un décès au domicile. Dans celle d'Anderson et ses collègues [2003], on en dénombre également sept. Aucune de ces deux études ne mettent en cause le soin à domicile. Dans l'essai de Vokes et ses collègues [1989], la mort d'un patient au domicile tout en recevant son troisième cycle de chimiothérapie aurait pu tout aussi bien se passer à l'hôpital. L'autopsie n'a pas révélé de résultats significatifs autres que son cancer du larynx connu, et l'arythmie cardiaque a été estimée être la cause probable du décès. Ce que l'on retient c'est que plusieurs auteurs concluent que la chimiothérapie à domicile est faisable [Lowenthal \& al., 1996, Borras \& al., 2000, Virally \& al. 2003, Anderson \& al. 2003, Lewden-Bernadac \& al. 2008 ].

Cependant, beaucoup de ces études mettent en évidence des conditions de réalisation très encadrées. Des critères de sélections stricts sont employés pour décider des patients inclus dans les études randomisées. Cela tendait à montrer que tous les patients devant recevoir des chimiothérapies n'étaient pas a priori considérés comme éligibles à ce type de prise en charge. On remarque que dans cinq études, les patients étaient traités ou visités chaque jour à

\footnotetext{
${ }^{4}$ 5-fluoro-uracile, Navelbine, Gemzar, Taxol, Herceptin, Fludarabine, Methotrexate, Hycamtin.

${ }^{5}$ Pouvant comprendre de la Gemcitabine, du Trastuzamab, de la Vinorébline, de la Fludarabine, du Topotecan, du Paclitaxel, de la Fluoro-uracile, du Methotrexate, de la Bléomycine, de l'Adrimyacine et de la Vincristine.
} 
domicile par des infirmières formées de façon spécifique. [DeMoss, 1980 ; Malone \& al., 1986; Lowenthal \& al., 1996 ; Borras \& al., 2001; Rishin et Matthews, 2001]. D'après ces études, on observe que le sur-encadrement des patients pendant l'étude par les professionnels de santé dans le suivi du traitement peut améliorer les conditions de leurs succès.

A travers l'étude d'Anderson et ses collègues [2003], nous constatons que le premier cycle de chimiothérapie a été réalisé à l'hôpital. De plus, trois essais évoquent certaines exigences (coordination, organisation, soins infirmiers efficaces, communication claire) pour que soit faisable la chimiothérapie à domicile.

Enfin, nous remarquons que plusieurs études suggèrent que la chimiothérapie à domicile améliorerait la qualité de vie du patient [Lowenthal \& al., 1996 ; Brown \& al., 1997 ; Rischin \& al., 2000 ; Borras \& al., 2000 ; Virally \& al., 2003 ; Anderson \& al., 2003 ; Réseau OCLE, 2007 ; Lewden-Bernadac \& al., 2008 ]. Malheureusement peu d'entres elles sont explicites en ce qui concerne la mesure de cette qualité de vie. Cinq de ces essais sous entendent que le taux de satisfaction des patients est plus élevé en ce qui concerne les soins de chimiothérapie à domicile qu'à l'hôpital. Seulement les études d'Anderson et ses collègues (2003) et celle du Réseau OCLE ont utilisé un questionnaire pour mesurer le niveau de qualité de vie pendant une chimiothérapie à domicile («Quality of Life », "Retentissement du mode de prise en charge sur votre vie quotidienne »).

\section{Conclusion}

Les données présentes ont tendance à démontrer que le traitement à domicile peut être administré de façon sécuritaire et entraîne peu de complications ou d'accidents graves, à condition qu'il y ait un critère de sélection des patients et qu'une bonne organisation des soins soit garantie. La plupart des auteurs évoquent une meilleure qualité de vie pour le patient dans les soins de chimiothérapie à domicile mais ces affirmations sont rarement étayées par des preuves (questionnaire de qualité de vie).

Les conditions de l'organisation des ces chimiothérapies à domicile ont été reprises dans les recommandations de la réglementation de l'ANAES (2005) qui stipule que le patient doit pouvoir bénéficier d'une prise en charge coordonnée de nature à assurer la continuité des soins et la permanence 24 heures sur 24, que la réalisation du 1er cycle doit être faite à l'hôpital dans la mesure où elle permet une première appréciation de la tolérance globale à court. Outre le fait que ces possibilité ne sont considérées comme réunies que dans le cadre d'une Hospitalisation à Domicile ou d'un Réseau de soin, certaines molécules anticancéreuses actuelles ne peuvent être administrées qu'à l'hôpital (seules les molécules sur la liste de rétrocession peuvent être administrées à domicile). De plus, certains états de santé exclu la possibilité de prise en charge extra-hospitalière (ex: patient présentant un risque d'insuffisance rénale aiguë).

La chimiothérapie est donc praticable avec une organisation et une coordination des soins adéquates. Le rapport d'étape du groupe de travail sur le développement de la chimiothérapie à domicile (direction de l'hospitalisation ou d'organisation des soins, ministère délégué à la santé, septembre 2001) spécifiait qu'en plus des différentes modalités de coordination, il fallait que l'état et l'environnement du patient soit acceptable pour qu'il puisse être pris en charge à domicile. Il ne s'agit pas dans ce cas d'un restriction d'ordre médical.

On peut donc conclure que le moindre développement des solutions à domicile n'est pas lié à une crainte objective quant à la moindre qualité des soins.

\section{Bibliographie}


INCA (2009), Situation de la chimiothérapie des cancers en 2009, p7.

Direction générale de la Santé, les 70 mesures du Plan Cancer, 2003.

Haute Autorités de Santé, Analyse comparée de la chimiothérapie anticancéreuse administrée à l'hôpital ou prise en charge à domicile: aspects économiques et organisationnels, Juin 2005.

Vokes EE, Schilsky RL, Choi KE, Magid DM, Guarnieri CM, Whaling SM, et al. A randomized study of inpatient versus outpatient continuous infusion chemotherapy for patients with locally advanced head and neck cancer. Cancer 1989;63(1):30-6.

Rischin D, White MA, Matthews JP, Toner GC, Watty K, Sulkowski AJ, et al. A randomised crossover trial of chemotherapy in the home: patient preferences and cost analysis. Med J Aust 2000; 173(3):125-7.

Borras JM, Sanchez-Hernandez A, Navarro M, Martinez M, Mendez E, Ponton JL, Espinas JA, Germa JR. Compliance, satisfaction, and quality of life of patients with colorectal cancer receiving home chemotherapy or outpatient treatment: a randomised controlled trial. BMJ 2001; 322(7290):826

Lowenthal RM, Piaszczyk A, Arthur GE, O’Malley S. Home chemotherapy for cancer patients: cost analysis and safety. Med J Aust 1996;165(4):184-7.

Brown DF, Muirhead MJ, Travis PM, Vire SR, Weller J, Hauer-Jensen M. Mode of chemotherapy does not affect complications with an implantable venous access device. Cancer 1997; 80(5):966-72.

DeMoss CJ. Giving intravenous chemotherapy at home. Americal Journal of Nursing, 1980;10(12):2188-9.

Virally J, Fouques Duparc V, Lazzerrini C, Boiron C, Jagot JL, Mathieu M. Chimiothérapie à domicile du cancer bronchique. Etude rétrospective sur cinq ans. Rev Mal Respi $2003 ; 20: 683-9$

H Anderson, JM Addington-Hall, MD Peake, J McKendrik, K Keane and N Thatcher. Domiciliary chemotherapy with gemcitabine is safe and acceptable to advanced non-small-cell lung cancer patients: results of a feasibility study. British Journal of Cancer (2003) 89, 2190 - 2196.

Réseau territorial Saint Nazaire (OCLE). Chimiothérapie à domicile, évaluation à 3 ans. 2007.

B Lewden-Bernadac, M Courant-Menanteau, G Perrocheau, V Barbarot, P Thomare. Chimiothérapie à domicile et réseau ville-hôpital : expérience du réseau Onco Pays-de-la-Loire. Bull Cancer 2008 ; 95 (5) : 543-9.

Direction de l'hospitalisation ou d'organisation des soins, ministère délégué à la santé, septembre 2001. 


\section{ANNEXE 4 : \\ RECHERCHE DE LITTÉRATURE SUR LES EFFETS DE SEUIL CRITIQUE DES VOLUMES DE CHIMIOTHÉRAPIE PRATIQUÉES PAR LES INFIRMIÈRES}

Nous nous interrogeons ici sur la pertinence des notions de « seuil critique» et d' « effet de routine ». Nous parlerons de "seuil critique» pour définir le nombre de séances de chimiothérapie en dessous duquel l'IDE pourrait perdre en qualification. La routine, elle, est définie comme une habitude normée à agir [3CERIS]. Ces habitudes peuvent être personnelles et organisationnelles. L' " effet de routine » sera défini dans cette étude comme l'impact de la perte de vigilance lié à une pratique trop régulière.

La chimiothérapie à domicile fait intervenir plusieurs acteurs. Son organisation complexe et demandant une rigueur irréprochable pour garantir le maximum de sécurité au patient, nécessite une qualification particulière. Face à la diversité et à la durée des traitements anticancéreux, ainsi qu'aux différentes compétences des infirmières, nous avons voulu explorer la pertinence de la notion de seuil critique en terme de pratique de chimiothérapie par infirmière en fonction du temps. Au delà du processus de qualification, faut-il s'assurer que l'infirmière pratique de façon régulière ou, peut-on laisser l'IDE (Infirmière Diplômée d'Etat) exercer seulement avec une qualification validée sans mise à jour des connaissances et/ou compétences? De manière plus générale, ce travail a pour but de faire un état des lieux de l'effet du volume des pratiques sur la sécurité des chimiothérapies.

\section{La chimiothérapie à domicile, hypothèses et contraintes}

Nos hypothèses de départ sont liées aux faibles volumes que nous avons pu observer quant à l'exercice de la chimiothérapie à domicile. Nous nous interrogeons sur l'obstacle que pourrait provoquer la faiblesse de cette pratique.

\subsection{Hypothèses à tester}

Nous avons émis les différentes hypothèses concernant les facteurs influençant l'habilitation à pratiquer la chimiothérapie à domicile, que nous souhaitons confronté à la littérature.

H1 : La pratique irrégulière d'injections et de perfusions d'anticancéreux à domicile par les infirmières libérales n'est pas un obstacle à la pratique.

H2 : L'absence de Formation Continue n'est pas un obstacle à la chimiothérapie à domicile pour les infirmières libérales.

H3 : L'organisation de la chimiothérapie à domicile ( $1^{\circ}$ cure à 1 'hôpital, préparation de la molécule par le PUI, ...) réduit les exigences du soin comparé au milieu hospitalier.

H4 : Le rattachement à une structure (réseau, HAD) tient une place importante dans le processus d'encadrement. 
Ces hypothèses confrontent la réalité de l'organisation actuelle avec ce que la littérature produit sur les pratiques professionnelles, afin de lever l'obstacle potentiel relatif à la faiblesse des pratiques.

\subsection{Pratique de la chimiothérapie par les infirmières libérales}

La chimiothérapie anticancéreuse consiste en l'administration de médicaments cytotoxiques qui empêchent les cellules de croître et de proliférer. Elle est surtout utilisée dans le traitement du cancer pour détruire les cellules néoplasiques qui se développent de façon anarchique [4]. Ce traitement est souvent associé à d'autres stratégies antinéoplasiques comme la chirurgie ou la radiothérapie. Du fait de la toxicité cellulaire des produits utilisés, la rigueur dans les soins doit être importante. Le soin de chimiothérapie anticancéreuse à domicile est un acte demandant des compétences complexes. En effet, il faut avoir des connaissances en soins infirmières dans le domaine de la cancérologie (sémiologie, pharmacologie, techniques et risques liés aux traitements mis en œuvre), et connaître des techniques de communication en vue de mieux utiliser la relation d'aide lors de la prise en charge des patients à domicile [annexe 1].

$\mathrm{Au}$ regard des paramètres biologiques, et à l'exception de certaines molécules comme l'Herceptine巴 c'est le médecin qui donne le feu vert pour la cure de chimiothérapie. Une fois l'ordonnance réalisé par l'oncologue, la première cure aura lieu à l'hôpital, à côté de l'oncologue prescripteur, les séances suivantes pouvant être réalisées à domicile. Le patient ne devra pas revoir systématiquement son cancérologue.

Les traitements anticancéreux se sont diversifiés (molécules orales ou en intraveineuse, différents degrés de toxicité...) tant en milieu hospitalier qu'à domicile. Aussi pour adapter la réglementation à ces nouvelles pratiques, le décret du 6 octobre 1989 reconnaissait aux infirmières la possibilité d'accomplir des injections de produits anticancéreux au travers de montages d'accès vasculaires implantables mis en place par le médecin [6]. Cela ouvrait la voie aux infirmières d'administrer sans présence du médecin.

\subsection{Qualification des IDE}

Les soins de chimiothérapie à domicile sont réglementés par une formation supplémentaire pour tous les IDE ayant obtenu leur diplôme avant 1992. L'Arrêté du 20 décembre 2004 a fixé les conditions d'utilisation des anticancéreux injectables inscrits sur la liste prévue à l'article L. 5126-4 du code de la santé publique et précise [7]: " Préalablement à l'administration d'une chimiothérapie anticancéreuse à domicile, les infirmières doivent avoir suivi une formation spécifique prévue dans la circulaire DGS /OB n 381 du 2 mars 1990 ou dans le cadre de leur formation initiale » [8]. L'IDE reçoit une attestation «formation chimiothérapie » après avoir validé cette formation technique. Elle est par la suite habilitée à assurer les injections et perfusions de produits anticancéreux au domicile.

Pour les infirmières formées après 1992, une attestation spécifique de formation à la chimiothérapie n'est plus requise puisqu'une formation théorique sur la chimiothérapie fait partie intégrante du cursus général de formation des IDE [9].

Plusieurs structures, comme le Réseau Onco Poitou-Charentes, le Réseau Onco-Paca ou encore le Réseau Onconord-Ile de France, le Centre Léon Bérard à Lyon, offrent des formations spécifiques pour les soins chimiothérapiques à domicile ou en milieu hospitalier à tout autre professionnel médical ou paramédical le souhaitant. [10]. Celle-ci n'est pas obligatoire mais permet notamment à l'infirmière de s'auto-évaluer et d'actualiser ses 
connaissances, ce qui pourrait améliorer la qualité des soins du patient.

\subsection{Habilitation des IDE}

« On entend par personnel habilité, un personnel possédant les qualifications requises par les lois et règlements reconnus par son responsable fonctionnel, capable d'accomplir les tâches qui lui sont confiées. Au sein des établissements de santé, le concept d'habilitation reste encore complexe tant dans sa perception que pour sa mise en œuvre » [12].

D'un point de vue réglementaire, l'infirmière Diplômée d'Etat est habilité à administrer des traitements anticancéreux à domicile soit par l'obtention de "l'attestation chimiothérapie » validée par la DRASS, soit par la justification de son diplôme d'état acquis après 1992 [8,9].

\section{Evaluation des Pratiques Professionnelles et identifications des Dysfonctionnements.}

Chaque jour, un incident ou accident peut survenir sur le lieu professionnel. Un des objectifs premiers pour comprendre ce dysfonctionnement organisationnel, matériel ou humain, est d'en connaître la cause. C'est notamment l'une des fonctions de l'EPP (Evaluation des Pratiques Professionnelles). Depuis la loi du 13 août 2004, tous les médecins, quelles que soient leurs modalités d'exercice, sont soumis à une obligation d'évaluation de leurs pratiques professionnelles [1]. Cette définition insérée au Code de Santé Publique par le décret EPP précédemment cité, a pour but une amélioration continue de la qualité des soins et du service médical rendu [11].

Cette obligation n'est pas, à ce jour, étendue aux autres professionnels de santé exerçant une profession médicale ou paramédicale. Cependant, ceux-ci sont impliqués dans la mise en place de l'EPP à travers leur obligation de formation continue (loi du 9 août 2004) selon laquelle " l'obligation de formation est satisfaite notamment par tout moyen permettant d'évaluer les compétences et les pratiques professionnelles » [2]. Cette formation continue obligatoire a pour finalité le perfectionnement des connaissances et l'amélioration de la qualité des soins. Qu'en est-il à ce jour de l'évaluation des compétences des infirmières administrant des produits anticancéreux à domicile?

\subsection{Littérature et EPP}

Nos recherches sur la qualité et la sécurité des chimiothérapies à domicile montraient que les critères d'éligibilité des patients et la formation spécifique des IDE avaient un rôle décisif dans la rareté des incidents majeurs [Note de synthèse sur la sécurité et la qualité de la chimiothérapie à domicile]. Après plusieurs recherches (base de données, études antérieures et moteurs de recherche), nous n'avons trouvé que deux études traitant des dysfonctionnements des IDE dans la chimiothérapie à domicile. L'étude du Réseau territorial de Saint-Nazaire (OCLE) parle notamment de problèmes de coordination et d'organisation freinant la bonne transmission de l'information [13]. Lewden-Bernadac et ses collègues signalent des dysfonctionnements concernant essentiellement le flux d'informations [14]. D'autres soins ont été étudiés (Transfusion sanguine, soins de réanimation...) du point de vue des pratiques. L'analyse de ces soins nous permettrons d'établir, par proximité, un début d'argumentation au sujet de l'évaluation des pratiques professionnelles dans l'administration de traitement anticancéreux à domicile. Trois études ont retenu notre attention.

D'après un article publié en 2008 par le CHU de Dijon, une analyse de la filière a été 
effectuée pour rechercher les problèmes et les dysfonctionnements liés au retard de délai d'admission des patients à l'UNV (Unité neurologique Vasculaire) dans sa phase intrahospitalière. La mise en place des EPP a incité l'ensemble des acteurs de la filière intrahospitalière de prise en charge des AVC* (SAMU, SRAU, scanner, brancardiers, biochimie, Neurologie, Rééducation) à évaluer son fonctionnement et ses résultats [15]. L'ensemble des intervenants (personnel soignant) a été sensibilisé et formé sur la base d'un protocole standardisé sur une période de neuf mois. L'EPP a ainsi démontré qu'il était possible d'améliorer l'efficience d'une filière uniquement par la sensibilisation et la formation ciblée des différents acteurs concernés, la reconnaissance de leur rôle et de leur travail, aboutissant à une amélioration indiscutable des soins, mesurable concrètement et valorisante pour les professionnels de santé.

L'étude menée par C.Le Niger au CHU de Brest au sujet de la transfusion sanguine a permis de repérer dans un premier temps les dysfonctionnements pour les infirmières qui étaient liés aux variations de pratiques : double détermination du groupe sanguin, contrôle ultime prétransfusionnel, surveillance du patient pendant la transfusion [16]. Par la suite, une formalisation des bonnes pratiques a été mise en place (protocoles), et les protocoles ont été diffusés. Selon ces mêmes auteurs, «l'application des protocoles sur le terrain dépend de la connaissance de ceux-ci par les acteurs du soin et la compréhension de la raison d'être des différentes exigences. La diffusion des protocoles implique une formation. Le taux de pratique déclarée totalement conforme à la réglementation est passé de moins de $10 \%$ avant à plus de $90 \%$ après $»$.

Une analyse prospective des dysfonctionnements en réanimation chez 928 patients a recueillis 273 dysfonctionnements (01/2005 à 09/2006) [17]. Un quart (25,3\%) était lié aux soins infirmières notamment par des erreurs fréquentes d'administration de médicaments. Selon cette étude, parmi tous les dysfonctionnements, 101 auraient pu être évités. Des actions ont donc été entreprises (note d'information, modification ou créations de protocoles de soins, entrevue ciblées avec les agents) pour réduire ces dérèglements. Face à la multiplicité et la technicité grandissante des soins infirmières en réanimation dont certains peuvent se trouver en cancérologie, il est important d'avoir une évaluation des pratiques professionnelles afin de pouvoir diminuer ces altérations et d'améliorer la qualité des soins.

L'EPP démontre qu'il est possible d'améliorer l'efficience d'une filière uniquement par la sensibilisation et la formation ciblée des différents acteurs concernés. Pour cela il semble nécessaire d'établir au préalable une formalisation des bonnes pratiques, c'est à dire de mettre en place des protocoles.

\subsection{La Routine : un danger dans la pratique.}

La routine des gestes dans l'administration de toute molécule peut également être source de problèmes dans les soins quotidiens. Christian Sicot ${ }^{*}$ cite " plus l'acte est routinier, plus il est important d'être attentif » [19]. Il signale que la répétition d'un geste peut diminuer l'attention et la rigueur dans la pratique, ce qui peut augmenter le risque d'incidents. La cancérologie demande plus de rigueur que d'autres spécialités (dermatologie, ophtamologie... ) du faite de la toxicité des traitements. Il semble donc important de faire attention aux routines reproduites par mimétisme et stéréotypie qui ne donnent aucune assurance de bien agir.

\footnotetext{
* Accident Vasculaire Cérébrale

* Chef de service de réanimation de l'hôpital d'Eaubonne-Montmorency (1974-2003); secrétaire général du SOU Médical groupe MACSF (1993-2008); directeur de la rédaction de la Revue Responsabilité (2003-2008) ; président de l'association La Prévention Médicale (2005-2008
} 
D'après l'étude de V.Buthion et ses collègues sur la transfusion sanguine [20], « la routine apparaît presque plus dangereuse que l'absence à la pratique régulière ». Cette étude suppose qu'après un certain nombre d'années, le personnel développe des habitudes de travail qui ne sont pas forcément en accord avec les règles de bonnes pratiques mais que personne ne remet en question. Un exemple bien précis est expliqué par l'un des coordinateurs régional de la DRASS ; une infirmière n'utilisait pas du sérum physiologique pour réactiver les anti-sérum qui sont déposés sur les cartes de contrôle lors du test d'agglutination. Cela faisait des années qu'elle utilisait de l'eau distillée. Malheureusement celle-ci provoque l'éclatement des globules rouges (hémolyse). L'infirmière ne voyait donc pas d'agglutination puisque effectivement il n'y avait pas d'agglutination, et jamais personne ne s'était rendu compte qu'elle faisait une erreur technique. Cet exemple extrême montre qu'une infirmière pratiquant rarement peut être plus attentive aux protocoles, alors qu'une infirmière peut réaliser le contrôle de manière machinal et utiliser un procédé non-conforme.

Ceci tend à montrer que les infirmières qui pratiquent plusieurs séances de chimiothérapie par jour, doivent peut-être redoubler de rigueur pour ne pas se laisser « absorber » par la routine.

\subsection{Existe-t-il un Seuil Critique?}

Le Réseau Territorial de Cancérologie Source à Lyon a réalisé 532 séances de chimiothérapie à domicile pendant l'année 2009, réparties sur 69 patients, mobilisant donc au minimum 69 infirmière, soit une moyenne de 7,7 injections par infirmière. Le Centre Léon Bérard à Lyon a réalisé en 200917866 séjours pour chimiothérapies en séance (Source ATIH) dans son hôpital de jour pour un effectif moyen de 24 infirmières soit environ 744 séances par infirmière et par an. Dès lors, le nombre moyen d'actes effectués par les infirmières (ères) libérales (ales) est-il suffisant pour ne pas être déqualifié? Peut-on considérer qu'une IDE ait un niveau de qualification acceptable si elle n'a administrée aucun traitement anticancéreux au cours de l'année ? La perte ou le gain de qualification est-il seulement lié au taux de pratique de séance par période?

La pratique régulière de la chimiothérapie à domicile pourrait être moins nécessaire qu'en milieu hospitalier du fait de la simplification des exigences [21].

Tous ne sont pas admissibles en fonction de leurs antécédents, de la molécule et de leur état clinique. En effet, La circulaire DGS/DH/AFS n 98-213 du 24 mars 1998 relative à l'organisation des soins en cancérologie dans les établissements d'hospitalisation publics et privés indique que les traitements comportant pour le malade des risques aigus, immédiats ou à terme différé (notamment certaines chimiothérapies) doivent être réalisés dans des structures de soins de référence, mais que certaines séances de chimiothérapie à risque réduit, non définies par les textes, peuvent néanmoins être réalisées dans le cadre des soins à domicile [22].Ces conditions rejoignent les critères de sélection à la chimiothérapie à domicile évoqués par la DHOS* [23].

La première cure de chimiothérapie est faite à l'hôpital et pour les autres cures qui seront faites à domicile, la molécule reste la même. Cela permet de déceler d'éventuels risques et de vérifier la tolérance au traitement.

La préparation sous contrôle des molécules anticancéreuses est faite par des PUI*. l'infirmière ne manipule aucune préparation des traitements cytotoxiques. Son rôle se concentre plus précisément dans l'administration du traitement, ce qui réduit considérablement le risque d'accident.

\footnotetext{
* DHOS : Direction de l'Hospitalisation et de l'Organisation des Soins

* PUI : Pharmacies à Usage Intérieur
} 
Dans la littérature, la notion de «seuil critique » (c'est à dire le nombre d'acte en dessous duquel le professionnel perd en qualification) dans la pratique de la chimiothérapie à domicile n'est pas évoquée. L'offre très disparate entre les établissements conventionnels et le domicile au niveau des chimiothérapies (Sources ATIH, moins d'1\% à domicile) peut laisser penser que le risque de déqualification est alors plus présent à domicile. Mais les moindres exigences établies par 1'ANES (critères d'éligibilité de la chimiothérapie anticancéreuse à domicile, 2003) permettant aux infirmières de pratiquer à domicile nous laisse aussi envisager qu'il n'existe pas de seuil critique.

\section{Conclusion :}

La pratique faible et irrégulière dans l'administration de traitement cytotoxique chez les infirmières libérales par rapport aux infirmières en milieu hospitalier, ne semble pas être un frein pour continuer d'exercer, ni un problème majeur pour la qualité des soins. L'IDE qui n'a pas suivi de mise à jour de ses connaissances ou de formation continue depuis son habilitation (formation spécifique ou diplôme post 1992), n'est pas interdite de pratiquer la chimiothérapie à domicile.

On peut simplement conclure que le besoin de mise à jour des connaissances peut apparaître à la mise sur le marché de nouvelles molécule dont les effets à l'administration peuvent modifier la pratique. Le rattachement à des structures comme les HAD et les réseaux peut permettre de diffuser rapidement toutes les informations nécessaires et le cas échéant, organiser les formations nécessaires pour le mise à jour des connaissances.

\section{Bibliographie :}

[1] Art 14 Loi n 2004-810 du 13 août 2004 relative à l'assurance maladie (publiée au Journal Officiel du 17 août 2004).

[2] Art. L.4382-1, LOI n 2004-806 du 9 août 2004 relative à la politique de santé publique (publiée au Journal Officiel du 11 août 2004).

[3] Centre d'Etude et de Recherche en intervention Sociale. Émergence d'une culture, déclin d'une profession Soigner et prévenir au Sénégal et en Côte d'Ivoire, Abdou Salam Fall, Laurent Vidal. UNIVERSITÉ DU QUÉBEC EN OUTAOUAIS, Série Recherche no. 38 ISBN : 2-89251-252-2 ; Mai 2005.

[4] Swedish Council on Technology Assessment in Health Care (SBU). Chemotherapy for cancer: a critical review of the literature. Volume II. Stockholm, Suède : SBU; 2001. Rapport no 155/2.

[5] INCA (2009), Situation de la chimiothérapie des cancers en 2009, p8.

[6] Décret n89-723 du 6 octobre 1989 modifiant le décret n 84-689 du 17 juillet 1984 modifié relatif aux actes professionnels et à l'exercices de la profession d'infirmier. Journal Officiel 7 octobre 1989.

[7] Arrêté du 20 décembre fixant les conditions d'utilisation des anticancéreux injectables inscrits sur la piste prévu par l'article L.5126-4 du code de la santé publique. Journal officiel 23 décembre 2004.

[8] Direction générale de la santé. Circulaire DGS n³81 du 2 mars 1990 relative à la formation continue des infirmiers participants aux chimiothérapies anticancéreuses. Paris : DGS ; 1990.

[9] Agence national d'accréditation et d'évaluation en santé. Critères d'éligibilité des patients en chimiothérapie anticancéreuse à domicile. Consensus formalisé de professionnels. Saint-Denis p 21 : ANAES ; 2003.

[ 10 ] Formation à $1 \mathrm{a}$ chimiothérapie à domicile accessible sur: http://www.oncopaca.org/fr/professionnels/formations, $\quad$ http://www.onconord.org/formations- 
36_39.html, http://www.onco-poitou-charentes.fr/fr/soignant_social.

[11] Décret $\mathrm{n}^{\circ}$ 2005-346 du 14 avril 2005 relatif à l'évaluation des pratiques professionnelles (publiée au Journal Officiel du 15 avril 2005).

[12] Cabaud J.-J. Drass île-de-France, France Formation, habilitation et suivi des compétences. Transfusion cliniques et biologique ISSN 1246-7820 Congrès Société française de transfusion sanguine SFTS. Congrès national $\mathrm{n}^{\circ} 23$, Tours, France (02/07/2007) 2007, vol.14, $\mathrm{n}^{\circ} 1$ (162 p.) [Document : 5p] (19 ref), pp. 152-156.

[13] Réseau territorial Saint Nazaire (OCLE). Chimiothérapie à domicile, évaluation à 3 ans. 2007.

[14] B Lewden-Bernadac, M Courant-Menanteau, G Perrocheau, V Barbarot, P Thomare. Chimiothérapie à domicile et réseau ville-hôpital : expérience du réseau Onco Pays-de-la-Loire. Bull Cancer 2008 ; 95 (5) : 543-9.

[15] Accidents Vasculaire cérébraux : démarche qualité = mortalité réduite, accessible sur

http://web.reseau-chu.org/articleview

[16] C. Le Niger and L. Lepiouf, Evaluation des pratiques professionnelles sur les dysfonctionnements médicaux et paramédicaux repérés au cour du processus transfusionnel au CHU de Brest ; Transfusion Clinique et Biologique, Volume 14, Issue 5, November 2007, p. 457-463

[17] Analyse prospective des dysfonctionnements en réanimation, accessible sur http://www.srlf.org

[18] P. Bizouarn. Evidence-based medicine: methods and critics. Annales Françaises d'Anesthésie et de Réanimation 26 (2007) 334-343.

[19] Sécurité du patient. La « révolution culturelle » à venir. Bulletin de l'Ordre, vol nº, Juin-Juillet 2005, accessible sur www.conseil-national.medecin.fr

[20] V. Buthion, R. Remonay, C. Denechaud. Organisation de la transfusion sanguine thérapeutique : études des modalités alternatives de la transfusion sanguine thérapeutique organisable en ambulatoire. Rapport de recherche, Décembre $2009: 175$.

[21] ANAES. Critères d'éligibilité des patients à une chimiothérapie anticancéreuse à domicile. Argumentaire ; Septembre 2003.

[22] Direction générale de la santé, Direction des hôpitaux. Circulaire DGS/DH/AFS n 98-213 du 24 mars 1998 relative à l'organisation des soins en cancérologie dans les établissements d'hospitalisation publics et privés. Paris : DGS ;1998.

[23] Direction de l'hospitalisation ou d'organisation des soins, ministère délégué à la santé, septembre 2001.

[24] INCA (2009), Situation de la chimiothérapie des cancers en 2009, p10

[25] Donnée de la base ATIH/PMSI 2008 ; HAD (mode de prise en charge principal : chimiothérapie) 
Page 89 sur 122 


\section{ANNEXE 5 : \\ ENQUÊTE AUPRÈS DES INFIRMIÈRES LIBERALES SUR LA PRATIQUE DE LA CHIMIOTHERAPIE A DOMICILE}

Nous avons souhaité, dans une de ces zones, le Rhône, interroger les infirmières libérales. La visée de cette étude est d'appuyer le cadre d'analyse de l'étude générale en s'intéressant à la réceptivité face à la possibilité éventuelle de développer et d'augmenter le nombre de séances de chimiothérapie à domicile. Il s'agit d'une étude pilote, qui n'a donc pas cherché une représentativité statistique.

\section{Cadre conceptuel pour la construction du questionnement et de l'échantillon}

Dans un premier temps, nous avons cherché à donner un ordre d'idée du nombre de séances de chimiothérapie que chacune pratique régulièrement à domicile. Dans un second temps nous avons cherché à savoir si les infirmières accréditées seraient favorables au développement de la chimiothérapie à domicile. Enfin, nous avons essayé de déchiffrer les éventuelles difficultés rencontrées et les améliorations proposées par les infirmières.

Les hypothèses de travail que nous souhaitions tester sont les suivantes :

1) Les infirmières pratiquant la chimiothérapie à domicile seraient à priori enclines à augmenter leur nombre de séances de chimiothérapie à domicile si on le leur proposait.

2) Les incidents à domicile restent faibles et sont sans complication grave. Par ailleurs, ils ne sont pas un facteur de crainte pour les infirmières.

3) La chimiothérapie à domicile se pratique autant en milieu rural qu'en milieu urbain.

Le recueil de données a été réalisé grâce à un questionnaire semi-qualitatif appliqué à tous les cas. Le questionnaire (annexe 6) comporte des questions fermées ayant pour but un dénombrement et d'autre part un recueil d'opinions grâce à des questions ouvertes.

Les individus ont été recrutés parmi les infirmières figurant dans les bases de données du réseau Source de cancérologie de Lyon depuis sa création (570 individus) ou dans l'annuaire téléphonique, sans aucune information sur leur éventuelle habilitation à administrer des produits cytotoxiques, avec une répartition respective de 3/4, 1/4. L'inscription au réseau Source entraîne une probabilité plus forte d'être qualifiée pour pratiquer la chimiothérapie, mais ce n'est pas systématique, car le réseau prend également en charge d'autres soins ne nécessitant pas cette qualification (nutritions parentérales, prises en charge de la douleur, soins palliatifs...). La prise en compte d'infirmières non recensées dans le réseau avait pour objectif de recueillir d'autres avis que ceux des IDE déjà impliquées dans les prises en charge.

Le nombre de chimiothérapies pratiquées par année au réseau Source est faible au regard du nombre d'infirmières potentiellement disponible (532 séances de chimiothérapie en 2009). Au regard de cette très faible demande à domicile, nous en déduisons qu'il est fort probable que ces infirmières pratiquent peu ou pas du tout. Afin d'avoir un échantillon assez représentatif concernant les infirmières réalisant des chimiothérapies à domicile, nous avons pris un nombre d'individus plus important chez les infirmières inscrites au Réseau Source que chez les infirmières libérales sélectionnées dans le bottin téléphonique. L'enquête s'est déroulée sur le département du Rhône.

Afin de sélectionner nos individus, nous avons utilisé une table du hasard. Un total de 90 infirmières (sur 570) ont été choisies parmi tous les participants au Réseau Source du CLB, et 
30 infirmières (sur 1180) parmi la rubrique «infirmière libérale » dans l'annuaire téléphonique.

Au total, l'ensemble des questionnaires a été envoyé aux 120 infirmières avec une lettre de présentation (annexe 6) reprenant le but de l'enquête. Les infirmières devaient remplir le questionnaire et le renvoyer avec l'enveloppe pré-timbrée jointe dans le courrier.

Le taux de retour a été de 50 (41 avec le Réseau Source et 9 avec l'annuaire téléphonique). Un numéro a été attribué à chaque infirmière de l'échantillon afin de garantir l'anonymat dans l'analyse des données.

\section{Résultats}

Cette partie est divisée en deux analyses statistiques. Une première qui est descriptive (dénombrements, mesures, graphiques) permettra notamment d'avoir une représentation du volume d'infirmières pratiquant la chimiothérapie à domicile et du volume d'actes pratiqués. La seconde partie qui est inférentielle (test $\mathrm{du} \chi^{2}$ ) permettra d'appliquer des tests d'indépendance, c'est à dire de montrer que deux critères sont indépendants. Nous essayerons de démontrer que le nombre d'années d'exercice n'a pas d'incidence sur le nombre d'incidents vécus, et que le nombre de séances de chimiothérapie ne dépend pas du milieu d'exercice.

\subsection{Analyse descriptive}

Dans un premier temps, nous observons selon les données récoltées $(n=50)$ qu'environ $3 / 4$ des infirmières libérales (74\% soit $37 \mathrm{IDE}, 62 \%$ dans le réseau et $12 \%$ en dehors) pratiquent la chimiothérapie à domicile.

Parmi celles-ci, la majorité (92\%) possède l'agrément. Ces conditions confirment bien la réglementation de l'arrêté du 20 décembre 2004 (" Préalablement à l'administration d'une chimiothérapie anticancéreuse à domicile, les infirmiers doivent avoir suivi une formation spécifique prévue dans la circulaire DGS /OB n 381 du 2 mars 1990 ou dans le cadre de leur formation initiale »). On remarque cependant que l'habilitation peut être attribuée si l'on justifie de plusieurs années d'exercice en cancérologie ou si l'on a suivi une formation à la chimiothérapie au cours d'ancien(s) poste(s). Trois infirmières sont concernées par ce cas.

Dans un second temps, nous constatons (voir le graphique ci-après) qu'approximativement $3 / 4$ (76\%) des IDE réalisent au minima 5 chimiothérapies à domicile par an et $46 \%$ en réalisent plus de 10. 
Graphique 1: Nombre de chimiothérapies par année chez les infirmières agréées.

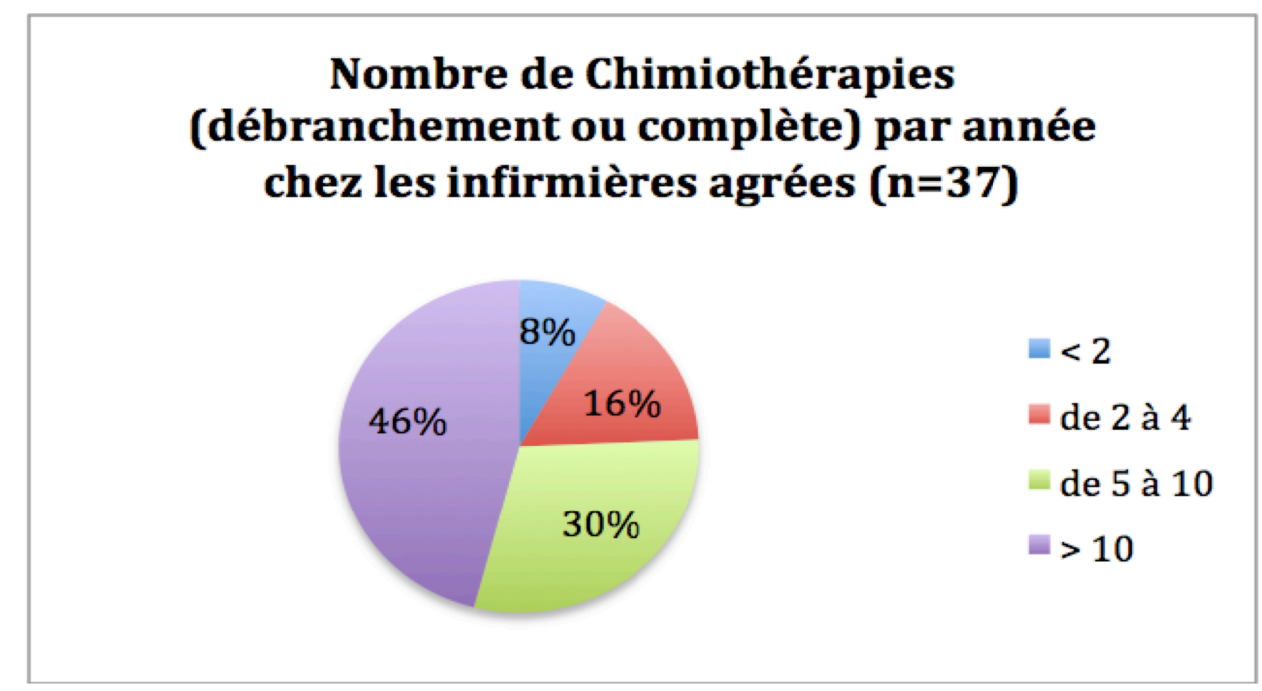

Malgré ces chiffres, l'activité reste très faible. D'autant plus que plusieurs IDE signalent une augmentation de la part des débranchements pour une diminution de la part des administrations de traitements anticancéreux.

Plus de $80 \%$ des infirmières pratiquant la chimiothérapie à domicile déclare une durée moyenne de soin inférieure à une heure, alors que les chimiothérapies qui sont susceptibles d'être confiées peuvent durer jusqu'à $1 \mathrm{~h} 30$.

Après analyse des questions ouvertes, nous notons que toutes les infirmières accréditées (37) souhaiteraient qu'on leur confie plus de prestations. Plusieurs déplorent que la formation qu'elles ont payée pour cela ne soit pas suivie de plus de sollicitations. Elles évoquent le fait que les hôpitaux et les cliniques ne « jouent pas le jeu » et « gardent les patients pour eux ».

Elles sont surtout sollicitées pour des surveillances ou des débranchements de perfusions posées en hôpital ou en clinique, alors qu'elles souhaiteraient que les hôpitaux "tiennent compte du fait qu'elles veulent faire plus de chimiothérapies complètes à domicile ».

D’une manière générale, nous constatons également qu'approximativement un quart $(24,3 \%)$ des infirmières ont eu un incident au cours de leur pratique. La majorité des incidents reste minime et concerne le matériel (voie veineuse bouchée ou problème de cathéter). Une seule complication est d'ordre plus symptomatologique : une infirmière a vécu un incident nommé " choc anaphylactique » c'est à dire une réaction allergique violente provoquant une forte perturbation de la circulation sanguine.

En ce qui concerne la rémunération, nous observons chez les infirmières libérales accréditées que $51 \%$ sont moyennement satisfaites et $38 \%$ décrivent la tarification de leur prestation comme insuffisante (graphique 2). Pour celles qui considèrent que la rémunération est insuffisante, elles vont jusqu'a émettre des contradictions dans la nomenclature des actes infirmiers : « une chimiothérapie en perfusion de moins de 1h est côté AMI10 alors qu'une perfusion standard est côté AMI12, c'est paradoxal ». 
Graphique 2 : Représentation des perceptions remuneration de la chimiothérapie à domicile

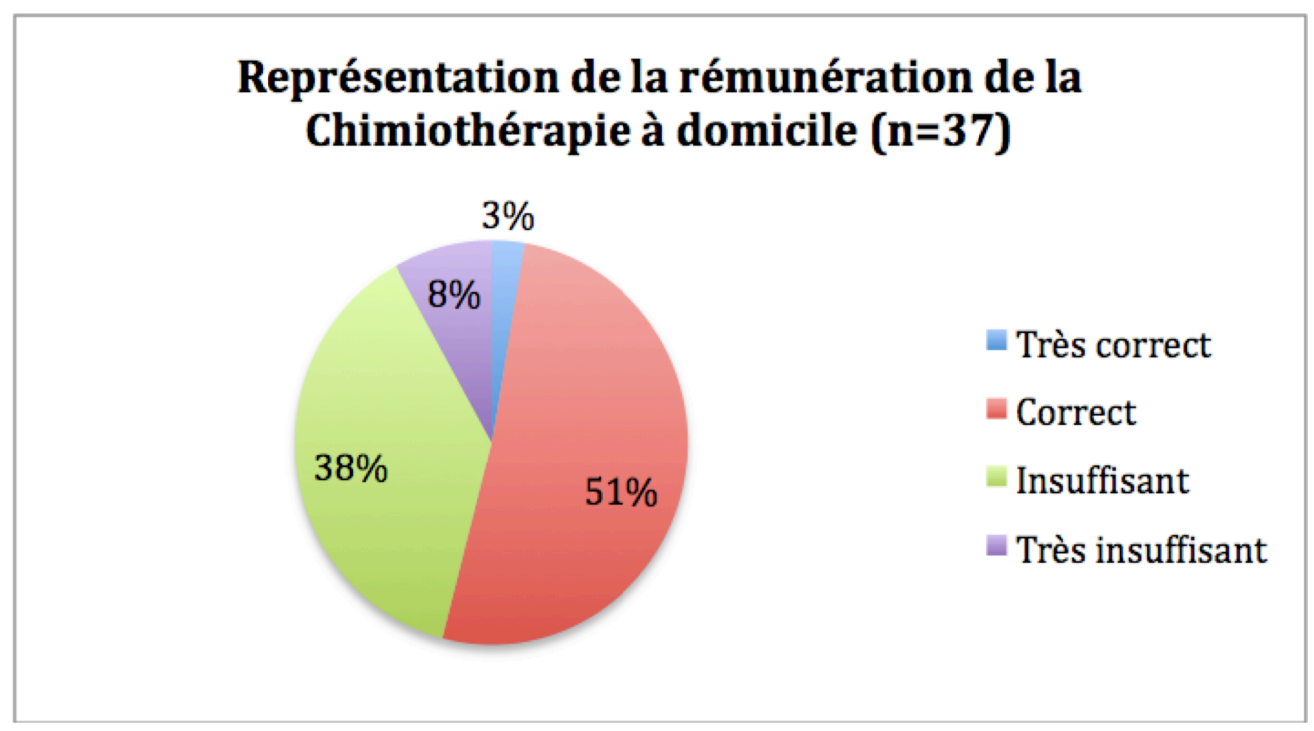

Nous avons aussi regroupé les différentes difficultés émises par les infirmières concernant l'organisation de la chimiothérapie à domicile. Les principales idées pour améliorer cette pratique sont au nombre de cinq (la première étant la plus citée), et sont les suivantes :

1) Une meilleure coordination et communication entre les différents acteurs de soins (Hôpital/libéral)

2) Plus d'information sur l'état clinique, environnemental et psychologique du patient (pathologie, traitement, caractère psycho-social...) car non accès au dossier médical.

3) Avoir plus d'information concernant les nouvelles molécules et les nouveaux protocoles.

4) Une meilleure organisation et gestion du matériel au domicile du patient, notamment des déchets.

5) Plus de formation continue.

Les mêmes propositions (sauf pour la formation continue) sont citées pour promouvoir les soins à domicile en général. Les actions de coordination, base centrale de l'organisation et donc de la réalisation des soins a domicile, sont dans l'ensemble assez critiquées. Une bonne partie considère les actions de coordinations comme moyennes, insuffisantes voire très insuffisantes. On peut se demander si le manque de coordination est un manque de compétences ou s'il y a un manque d'investissement de la part de certains acteurs.

\subsection{Analyse inférentielle}

Le test d'indépendance du $\chi^{2}$ permet de contrôler l'indépendance de deux caractères dans une population donnée. Nous avons voulu dans un premier temps démontrer l'indépendance entre le nombre d'incidents vécu et le nombre d'années d'exercice puis l'indépendance entre le nombre de séances de chimiothérapies et le milieu d'exercice.

\subsubsection{L'incident et l'ancienneté}

Nous dénombrons 9 infirmières ayant vécu un incident et 28 n'en n'ayant pas vécu. Parmi 
celles ayant eu un incident, nous constatons qu'un tiers $(n=3)$ pratiquaient la chimiothérapie depuis moins de 5 ans. La proportion des infirmières pratiquant depuis moins de 5 ans et n'ayant pas eu d'incident est quasiment de même ordre $34,61 \%(n=9)$. Nous avons donc voulu vérifier l'indépendance de ces deux critères, pour montrer que le nombre d'années d'exercice n'a pas d'incidence sur le nombre d'incidents vécus.

Soit les deux hypothèses suivantes :

H0 : Le fait d'avoir vécu un incident ne dépend pas du nombre d'années d'exercice (critère indépendant).

H1 : Le fait d'avoir vécu un incident dépend du nombre d'années d'exercice (critère lié).

Dans nos recherches nous avons noté que le Réseau Territorial de Cancérologie Source à Lyon avait réalisé 532 séances de chimiothérapie à domicile pendant l'année 2009, réparties sur 69 patients, mobilisant donc au minimum 69 infirmières, soit une moyenne de 7,7 injections par infirmière par an. Le Centre Léon Bérard à Lyon avait réalisé 17866 séjours pour chimiothérapie en séance (Source ATIH) dans son hôpital de jour pour un effectif moyen de 24 infirmière soit environ 744 séances par an et par infirmière. Au regard du faible volume de séances proposées à domicile, nous avons choisi arbitrairement une moyenne de 5 années d'exercice pour qu'une infirmière à domicile soit expérimentée, soit environ une cinquantaine d'actes (nombre moyen d'injections par infirmière participante au réseau Source multiplié par cinq ans).

Grâce aux données collectées dans l'enquête, il est possible de réaliser le tableau suivant.

\begin{tabular}{|l|l|l|l|}
\hline Valeurs observées & Incidents & Absence d'incidents & Total \\
\hline$<5$ années d'exercice & 3 & 9 & 12 \\
\hline$>5$ années d'exercice & 6 & 19 & 25 \\
\hline Total & 9 & 28 & 37 \\
\hline
\end{tabular}

Dans ce tableau, nous retrouvons les fréquences expérimentales mais aussi les totaux par lignes (12 infirmières ont moins de 5 années d'exercice contre 25 infirmières qui ont 5 ou plus de 5 ans d'exercices) et par colonnes ( 9 ont vécu un incident contre 28 qui n'en n'ont pas vécu). Il est alors possible de calculer les valeurs "théoriques» en se servant des valeurs expérimentales. La manière pour y parvenir est décrite dans le tableau suivant :

\begin{tabular}{|l|l|l|l|}
\hline Valeurs théoriques & Incidents & Absence d'incidents & \\
\hline$<5$ années d'exercice & $\left(9^{*} 12\right) / 37=2,92$ & $\left(28^{*} 12\right) / 37=9,08$ & 12 \\
\hline$>5$ années d'exercice & $(9 * 25) / 37=6,08$ & $\left(28^{*} 25\right) / 37=18,92$ & 25 \\
\hline & 9 & 28 & 37 \\
\cline { 2 - 3 } & & &
\end{tabular}

Pour déterminer le $\chi^{2}$ observé pour chaque échantillon de l'expérience, on applique la formule suivante :

$\chi^{2}=(\text { fréquence observée }- \text { fréquence théorique })^{2} /$ fréquence théorique

Nous obtenons donc le tableau suivant : 


\begin{tabular}{|l|l|l|l|}
\hline & Incidents & Absence d'incidents & Total \\
\hline $\begin{array}{l}<5 \text { années } \\
\text { d'exercice }\end{array}$ & $(3-2,92)^{2} / 2,92=0,0022$ & $(9-9,08)^{2} / 9,08=0,0007$ & 12 \\
\hline $\begin{array}{l}>5 \text { années } \\
\text { d'exercice }\end{array}$ & $(6-6,08)^{2} / 6,08=0,0011$ & $(19-18,92)^{2} / 18,92=0,0003$ & 25 \\
\hline Total & 9 & 28 & 37 \\
\hline
\end{tabular}

Pour tirer une conclusion sur la dépendance (H1) ou (H0), on somme tous les $\chi^{2}$ observés :

$\chi^{2}=0,0022+0,0007+0,0011+0,0003=0,0043$

On compare par la suite cette valeur globale à une valeur des tables. Cette table est une table à double entrée. L'entrée en ligne nécessite de connaître les degrés de libertés de l'expérience. Il se calcule de la manière suivante: (k-1).( $\mathrm{r}-1)$ avec $\mathrm{k}$ le nombre de colonnes et $\mathrm{r}$ le nombre de lignes. L'entrée en colonne est déterminée par l'expérimentateur. C'est en effet lui qui détermine l'intervalle de confiance du test.

Dans notre exemple, il n'y a que 2 lignes pour deux colonnes, soit (2-1)*(2-1) degrés de liberté. Nous prendrons un intervalle de confiance à $95 \%$ (c'est à dire un risque de se tromper de $5 \%$ ), la valeur de $\chi^{2}$ des tables est :

$\chi^{2}(1 \mathrm{ddl}, 0.95)=3,84$

Nous avons donc un $\chi^{2}$ observé de 0,0043. Cette valeur est inférieure à 3,84(valeur des tables). L'hypothèse $\mathrm{H} 0$ est donc acceptée.

Cela implique qu'avoir vécu un incident ne dépend pas du nombre d'années d'exercice (ancienneté). Une infirmière agrée depuis peu n'est pas forcément sujette à avoir plus d'incidents que son " aînée "; d'autant plus que selon notre échantillon, nous observons deux fois plus ( 6 contre 3$)$ d'incidents chez les infirmières ayant 5 années ou plus d'expérience que les infirmières pratiquant depuis moins de 5 ans.

La moyenne du nombre d'années d'exercice sans incidents est a peu près de 7 années $(n=28)$. Nous constatons alors que la proportion d'infirmières $(n=9)$ qui ont vécu un incident $(24,3 \%)$ pendant leur pratique n'est pas très significative ; il aurait été intéressant d'avoir un plus grand échantillon est de pouvoir comparer le nombre d'incidents vécu en fonction du nombre d'années d'exercice. Au regard du nombre important d'effets secondaires des produits cytotoxiques, avoir eu un incident non grave (sans complications majeurs pour le patient) en dix années de pratique par exemple ne semble pas alarmant.

\subsubsection{Volumétrie et milieu d'exercice}

Parmi les infirmières accréditées, nous en notons 16 pratiquant en milieu urbain contre 21 en milieu rural. Nous constatons que $31,25 \%(n=5)$ des infirmières exerçant en milieu rural font moins de 5 séances de chimiothérapie par an contre 19,05\% $(n=4)$ exerçant en milieu urbain. Nous avons donc voulu vérifier l'indépendance de ces deux critères, c'est-à-dire que le nombre de séance de chimiothérapies réalisé par les infirmières ne dépendait pas du lieu d'exercice.

Soit les deux hypothèses suivantes : 
H0: Le nombre de séances de chimiothérapie ne dépend pas du milieu d'exercice (critère indépendant).

H1 : Le nombre de séances de chimiothérapie dépend du milieu d'exercice (critère lié).

La question « Combien de chimiothérapies à domicile (débranchements ou complètes) faitesvous par année en moyenne ? » est constitué de 4 classes de réponses. Nous avons décidé de la regrouper en 2 classes $(<5$ et $\succ 5)$ pour traiter ce test $\chi^{2}$.

Grâce aux données collectées dans l'enquête, il est possible de réaliser le tableau suivant.

\begin{tabular}{|l|l|l|l|}
\hline Valeurs observées & milieu plutôt urbain & milieu plutôt rural & Total \\
\hline 5 séances/an & 5 & 4 & 9 \\
\hline$\succ 5$ séances/an & 11 & & \\
\hline Total & 16 & 17 & 28 \\
\hline
\end{tabular}

Dans ce tableau, nous retrouvons les fréquences expérimentales mais aussi les totaux par lignes ( 9 infirmières réalisant moins de 5 séances par contre 28 qui réalisent 5 ou plus par an) et par colonnes (21 qui pratiquent en milieu rural contre 16 qui pratiquent en milieu urbain).

Puis, il est possible de calculer les valeurs "théoriques" en se servant des valeurs expérimentales. La manière pour y parvenir est décrite dans le tableau suivant:

\begin{tabular}{|l|l|l|l|}
\hline Valeurs théoriques & milieu plutôt urbain & milieu plutôt rural & \\
\hline$<5$ séances/an & $\left(16^{* 9}\right) / 37=3,89$ & $(21 * 9) / 37=5,11$ & 9 \\
\hline$\succ 5$ séances/an & $\left(16^{*} 28\right) / 37=12,11$ & $(21 * 28) / 37=15,89$ & 28 \\
\hline & 16 & 21 & 37 \\
\hline
\end{tabular}

Pour déterminer le $\chi^{2}$ observé pour chaque échantillon de l'expérience, on applique la formule suivante :

$\chi^{2}=(\text { fréquence observée }- \text { fréquence théorique })^{2} /$ fréquence théorique

Nous obtenons donc le tableau suivant :

\begin{tabular}{|l|l|l|l|}
\hline & milieu plutôt urbain & milieu plutôt rural & \\
\hline$<5$ séances/an & $(5-3,89)^{2} / 3,89=0,317$ & $(4-5,11)^{2} / 5,11=0,241$ & 9 \\
\hline $\begin{array}{l}\succ \\
\text { séances/an }\end{array}$ & $(11-12,11)^{2} / 12,11=0,102$ & $(17-15,89)^{2} / 15,89=0,077$ & 28 \\
\hline & 16 & 21 & 37 \\
\hline
\end{tabular}

Pour tirer une conclusion sur la dépendance (H1) ou (H0), on somme tous les $\chi^{2}$ observés : 
$\chi^{2}=0,317+0,241+0,102+0,077=0,737$

On compare par la suite cette valeur globale à une valeur des tables. L'entrée en colonne est déterminée par l'expérimentateur. Nous prendrons un intervalle de confiance à $95 \%$ (c'est à dire un risque de se tromper de $5 \%$ ), la valeur de $\chi^{2}$ des tables est :

$\chi^{2}(1 \mathrm{dd} 1,0.95)=3,84$

Nous avons donc un $\chi^{2}$ observée de 0,737 . Cette valeur est inférieure à 3,84 (valeur des tables). L'hypothèse $\mathrm{H} 0$ est donc acceptée.

Le nombre de chimiothérapie ne dépend pas du milieu d'exercice. Les résultats semble indiquer une quantité à peu près similaire $(43,5 \%$ contre $56,5 \%)$ concernant les actes de chimiothérapie réalisés respectivement en milieu urbain et en milieu rural. Ainsi, le fait de travailler en milieu rural ne favorise pas l'augmentation du volume d'actes

\section{Discussion}

Dans notre enquête, une majorité (75\%) des infirmières accréditées n'a jamais vécu d'incidents pendant leur pratique. Une seule infirmière (sur 37) a vécu une complication grave (choc anaphylactique). Les autres accidents sont minimes et sans complications majeures pour le patient. Ces résultats montrent que la pratique actuelle n'a pas de raison d'être source d'inquiétude de la part des infirmières libérales.

Nous constatons également, à l'unanimité $(\mathrm{n}=37)$ des infirmières réalisant la chimiothérapie à domicile, le souhait d'augmenter le volume cette activité. Il ressort d'une manière générale une réelle envie de s'investir et de pratiquer plus, surtout d'avoir une prise en charge globale du patient ("je suis pour une augmentation des chimiothérapies à domicile car l'IDE est maintenant préparée pour y faire face », "je suis désireuse de faire des chimiothérapies à domicile avec une prise en charge globale du patient »). Comme nous le montre également les résultats, cette pratique pourrait se développer autant en milieu rural qu'en milieu urbain.

Environ 70\% (25 infirmières) considère que le nombre de séances de chimiothérapies a diminué ou stagné ces dernières années. Après la mesure $n^{\circ} 41$ du plan cancer («faciliter la chimiothérapie à domicile et plus généralement les soins à domicile). Notre enquête tend à montrer que le frein à ce développement ne vient pas des infirmières.

Certaines infirmières interrogées dénoncent une logique économique. Elles indiquent que l'instauration de la T2A en 2005 a changé le fonctionnement des établissements hospitaliers en termes d'activité. Elles évoquent le fait que les hôpitaux ou cliniques ne "jouent pas le jeu » et "gardent les patients pour eux». Ceci tend également à monter que l'encombrement des structures hospitalières ne semble pas aussi important que cela a pu être évoqué par certains acteurs, ou que ces derniers souhaitent se décharger d'autres chose que de cette prestation.

Enfin, un terme récurrent, celui du manque de coordination entre les différents acteurs de soins, est évoqué par la plupart des infirmières. Elles soulignent le manque de communication entre le milieu hospitalier et le milieu libéral. Elles ajoutent vouloir plus de relation et de disponibilité de la part des prestataires et des médecins. Les actions de coordination sont dans l'ensemble jugées moyennes voire insuffisantes. Ceci tend à nous interroger sur un manque de compétence en coordination ou un manque d'intéressement et d'investissement de la part des différents acteurs, et pose la question de la coordination de la prise en charge du patient. 


\section{ANNEXE 6 : \\ QUESTIONNAIRE A DESTINATION DES INFIRMIÈRES \\ LIBERALES}

Objet : courrier et questionnaire IDE.

Lyon, le 26/04/2010

Madame, Monsieur,

Nous faisons actuellement parti d'une équipe de recherche en sciences sociales (GATE) attachée au CNRS, à l'Université de Lyon, associée au Centre Léon Bérard et en relation avec le Réseau Source. Nous menons différentes études sur l'organisation des soins. Devant l'augmentation du nombre de chimiothérapies rendues nécessaires par l'accroissement de l'incidence des cancers, nous allons réaliser un travail portant sur l'organisation et les perspectives de la chimiothérapie anticancéreuse à domicile.

Pour cela nous avons établi un questionnaire afin de mieux connaître vos pratiques actuelles, les éventuelles difficultés rencontrées et vos souhaits pour le futur.

Vous serait-il possible de nous accorder un peu de votre temps pour y répondre, et de nous le renvoyer dans l'enveloppe pré timbrée ci-jointe.

En vous remerciant par avance de votre implication pour cette étude, veuillez agréer, Madame, Monsieur, l'expression de nos salutations distinguées.

Valérie BUTHION

Maître de Conférences des Universités
Anouar FANIDI

Infirmier Diplômé d'Etat

Etudiant Master Santé

Population 
Page 99 sur 122 


\section{Questionnaire}

Nom de la personne interrogée :

Adresse :

Nous vous informons que le traitement des résultats est anonyme.

Les questions sont recto-verso.

1) En quelle année avez-vous eu votre diplôme?

2) Exercez-vous :

Seule

En cabinet avec d'autres IDE

Autre organisation :

3) Avec quel type d'interlocuteur travaillez-vous ?

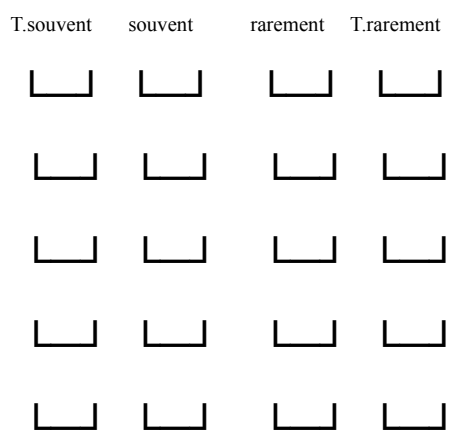

Autre type de structure

4) Milieu d'exercice principal ?

Milieu plutôt urbain

Milieu plutôt rural

5) Quels actes pratiquez-vous?

Prélèvements et injections

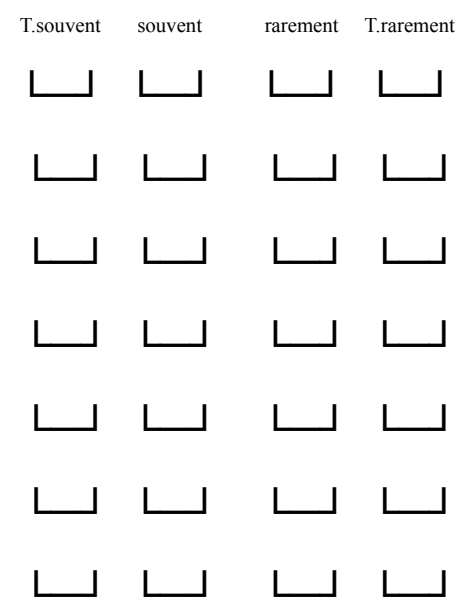

Administration traitement (IV \& per os) 
Soins spécialisés :

Entretien cathéter

Injections et perfusions par VVC
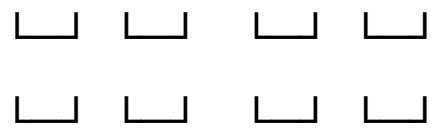

Traitement spécifique chez patient

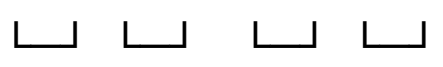

immunodéprimé ou cancéreux

6) Quels types de soins pratiquez-vous en cabinet ? (citez les plus courants)

7) Quelle est approximativement la proportion journalière de vos soins pratiqués en cabinet?

2 heures

4 heures

6 heures

8 heures

8) Quelle est la durée du soin le plus long que vous pratiquez à domicile ?

9) Vos suggestions pour améliorer les soins à domicile en générale ?

10) Comment jugez-vous les actions de coordination réalisés autour des patients recevant des soins à domicile en général?

11)Pratiquez-vous la chimiothérapie à domicile?

Oui

Non

Si non, pourquoi ? 
12) Possédez-vous l'attestation «Formation Chimiothérapie »?

Oui

Depuis combien de temps :

$$
\begin{aligned}
& <1 \text { an } \\
& 1-3 \text { ans } \\
& >\text { 3ans }
\end{aligned}
$$

\section{Non}

Si non, avez-vous suivi une formation à la chimiothérapie au cours de votre (vos) ancien(s) poste(s)?

Oui

Non

13) Si vous possédez-vous l'attestation « Formation Chimiothérapie », où l'avez-vous $\underline{\text { acquise ? }}$

14)Depuis combien de temps pratiquez vous des soins de chimiothérapie à domicile?

15)Combien de chimiothérapie à domicile (débranchement ou complète) faites-vous par année, en moyenne?

$$
\begin{aligned}
& <2 \\
& \text { de } 2 \text { à } 5 \\
& \text { de } 5 \text { à } 10 \\
& >10
\end{aligned}
$$

16) La plupart des soins de chimiothérapie que vous pratiquez ont tendance à être :

$<1$ heure

$>1$ heure

17) Vous sentez-vous à l'aise dans ce type de soins? 
beaucoup

assez

un peu

pas du tout

Si peu ou pas du tout, pourquoi ? .....

18) Avez-vous déjà eu un ou des incidents pendant une chimiothérapie?

Oui

Quel(s) type(s):

Non

19) Comment vos sollicitations pour des chimiothérapies ont-elles évolué ces derniers temps?

De combien :

En augmentation

En diminution

De combien :

En stagnation

20) Accepteriez-vous plus de soins de chimiothérapie à domicile?

Oui

Non

Si non, pourquoi ?

21) Comment jugez-vous la rémunération des actes infirmiers de la chimiothérapie à

domicile?

Très insuffisant

Insuffisant

Correct

Très correct

22) Vos suggestions pour améliorer la chimiothérapie à domicile ? 
Page 104 sur 122

\section{Pour tous}

\section{3) Commentaires libres:}


Page 105 sur 122 


\section{ANNEXE 7 : \\ COÛT POUR L'ASSURANCE MALADIE DE L'ADMINISTRATION DE LA CHIMIOTHÉRAPIE EN AMBULATOIRE}

Les études de coûts comparés hôpital versus domicile n'ont pas jusqu'à présent montré de façon probante la supériorité économique de l'une ou de l'autre solution pour l'assurance maladie. Des tarifications mouvantes, des informations sur les coûts incomplètes ou mal renseignées, n'ont pas permis d'avoir une vision claire de la situation. En 2006, la chimiothérapie réalisée en ambulatoire dans les hôpitaux de jour a été isolée dans le PMSI dans un GHM unique, le [28Z07Z Chimiothérapie en séance]. Ce dernier fourni depuis une base d'information permettant une certaine continuité dans l'analyse, le passage progressif à la T2A depuis 2005 obligeant les hôpitaux à renseigner le système d'information de façon plus fiable. L'Echelle Nationale des Coûts hospitaliers pour 2007, désormais disponible, permet de questionner les charges engagées pour ce séjour, et de les comparer aux autres modes d'administration.

Nous avons conduit l'analyse économique et prenant le point de vue de l'assurance maladie. Pour les tutelles financières du monde de la santé, coût et prix se confondent souvent. Le prix payé à un hôpital, un infirmier libéral, un prestataire de service constitue un élément du coût final de la prestation. Que le prix payé comporte une prestation « tout compris ", comme dans le cas d'une chimiothérapie réalisée en hôpital de jour, ou qu'il soit constitué d'une dotation globale de coordination d'une part et de facturation de prestations libérales d'autre part, comme dans le cas des réseaux, cela coûtera à l'assurance maladie l'addition des factures de tous les intervenants.

Le cancérologue, qui est en général rattaché à une structure hospitalière, détermine le traitement à administrer, et lui seul interviendra pour modifier les molécules et les dosages. Le patient le rencontrera régulièrement pour faire le point sur l'ensemble de son traitement et des effets secondaires liés à la toxicité des molécules. Le traitement va comporter plusieurs injections appelées «cures ». Une fois l'ordonnance réalisée, la première cure aura lieu à l'hôpital, à proximité de l'oncologue prescripteur, les séances suivantes ne nécessitant pas, à l'exception de certaines molécules, cette proximité. Un feu vert médical est requis avant l'administration de la plupart des cures, mais ne nécessite pas systématiquement un cancérologue. Un autre médecin, et notamment un généraliste, peut délivrer ce feu vert.

Quel que soit le lieu d'administration, la cure se déroulera selon le même protocole organisationnel. La veille ou l'avant-veille de la cure, le patient se rend au laboratoire d'analyse médicale pour une prise de sang, les résultats sont communiqués aux équipes médicales qui le suivent et un médecin doit, au regard de paramètres biologiques et (si besoin) de l'examen du patient, donner le feu vert pour le déroulement de la cure. Seule une molécule, l'Herceptine, ne nécessite pas de feu vert du médecin. La (ou les ) molécule(s) est (sont) alors commandée(s) à une PUI. Le patient se rend à l'hôpital de jour, ou l'équipe de soin à domicile (HAD ou Réseau) organise la venue de l'infirmière et l'acheminement de la molécule chez le patient. Le produit est administré par l'infirmière libérale. Le dossier du patient est mis à jour après retour de l'information sur le bon déroulement de l'administration du produit par l'IDE libérale.

L'injection d'une chimiothérapie peut être courte (une injection par seringue) ou longue (certaines chimiothérapies se diffusent sur $24 \mathrm{~h}$ ou plus). Les chimiothérapies intraveineuses qui sont réalisées à domicile sont des injections ou des perfusions qui ne dépassent pas une heure et demi. Lorsque les chimiothérapies sont très longues, il arrive que la perfusion soit 
posée à l'hôpital et que le patient rentre chez lui pour être débranché quelques heures plus tard ou le lendemain par une infirmière libérale.

Nous présenterons dans cette annexe le résultat détaillé de l'analyse des données financières dont nous avons pu disposer pour le réseau de cancérologie et les hôpitaux public, PSPH et privé. Les données concernant le réseau de cancérologie Source sont les données internes au réseau. Les données concernant l'hôpital sont les données retraitées à partir de l'échelle nationale de coûts. Le résultat de cette étude constitue une estimation.

\section{L'expérience d'un réseau santé en cancérologie}

Le réseau Source, Réseau Territorial de Cancérologie, créé en 2007, a pour vocation de prendre en charge tout patient atteint de cancer du début à la fin de la maladie, en partenariat avec les autres acteurs de santé. Le réseau peut assurer la coordination de soins depuis le diagnostic, en passant par les traitements curatifs comme la chimiothérapie, les soins de support et/ou les soins palliatifs. Le projet de ce réseau a été porté par le Centre Léon Bérard, Centre de Lutte Contre le Cancer de Lyon et un certain nombre de professionnels libéraux (médecins, infirmiers(ères), pharmaciens, biologistes) implanté sur les territoires 7, 8, 9 et 10 du SROS III de la région Rhône-Alpes (cf schéma 1). Ce territoire représente un bassin de population de près de 2 millions d'habitants dans des zones hétérogènes : urbaines (Lyon) et rurales (Monts du Lyonnais, Nord Beaujolais...).

\section{Figure 1: Territoire du réseau Source}

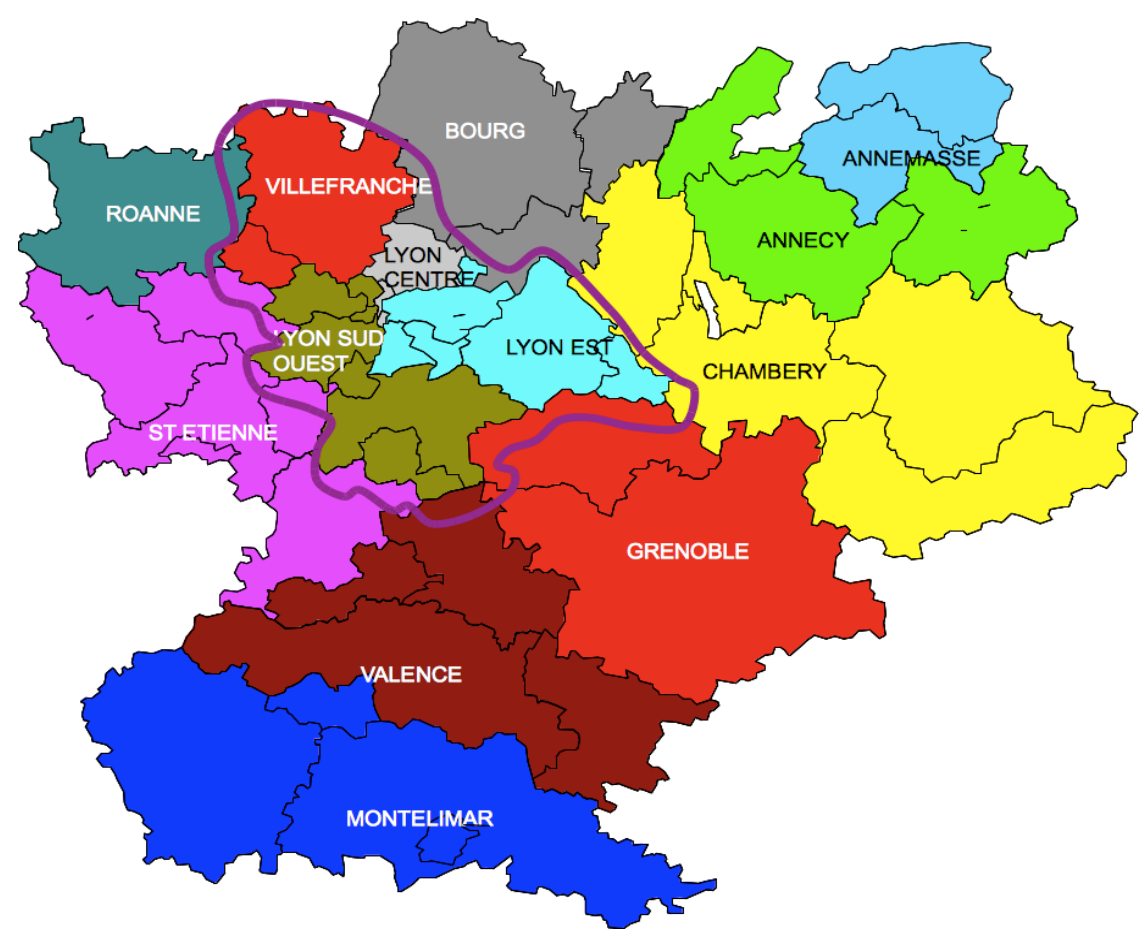

Le Réseau Source a été agréé en décembre 2007, il a commencé son activité en juin 2008 et les premiers patients ont été pris en charge à partir de septembre 2007. Dès le mois de novembre 2007, des chimiothérapies " entièrement réalisées à domicile » ont été réalisées à titre expérimental. Devant la demande importante des patients, la décision a été prise de faire de cette activité une priorité de développement pour l'année 2009. Ainsi, après avoir réalisé 35 séances de chimiothérapie en 2008, l'activité en 2009 s'est élevée à 532 séances de 
chimiothérapie réparties sur 69 patients, pour un total de 547 patients pris en charge. L'équipe a connu une montée en charge progressive pour atteindre 8 ETP (1 coordonnateur responsable administratif, 1 secrétaire, 3,5 ETP Infirmière, $1 / 2$ ETP médecin, 1 assistante sociale, 1 chauffeur-livreur) fin 2009.

Le réseau reçoit un budget de la part de l'Urcam (Union Régionale des Caisse d'Assurance Maladie) sur la dotation FIQCS (Fond d'Intervention pour la Qualité et la Coordination des Soins). Sur ce budget, le réseau doit payer les infirmières qui coordonnent les soins, la préparation par une PUI et la livraison des molécules de chimiothérapie. La ressource de base de l'activité du réseau est donc l'heure de coordination infirmière. Seront facturées directement à l'assurance maladie l'analyse de sang de contrôle préalable, la consultation du médecin traitant qui donnera le feu vert, les médicaments nécessaires dont les molécules de chimiothérapie, la prestation de l'infirmière qui administrera le médicament ainsi que ses frais de déplacement et le kit stérile de matériel permettant la perfusion.

\subsubsection{Analyse de l'activité du réseau Source pour l'année 2009}

2009 était la première année de plein exercice du réseau. Sur cette première année de montée en charge, le réseau a bénéficié de 31 mois équivalent temps plein d'infirmières (cf tableau 1)

Tableau 1: Composition de l'effectif des infirmières de coordination en 2009

\begin{tabular}{|c|c|c|c|c|c|c|}
\hline Effectifs payés sur 2009 & & $\begin{array}{c}\text { Nombre de } \\
\text { mois }\end{array}$ & $\begin{array}{l}\text { Temps de } \\
\text { travail }\end{array}$ & $\begin{array}{c}\text { Nombre de } \\
\text { mois }\end{array}$ & $\begin{array}{l}\text { date de } \\
\text { début de } \\
\text { contrat }\end{array}$ & $\begin{array}{l}\text { date de fin } \\
\text { de contrat }\end{array}$ \\
\hline \multirow[t]{6}{*}{ Infirmière } & Infirmière A & 12 & $91,43 \%$ & 11 mois & & \\
\hline & Infirmière B & 5 & $100 \%$ & 5 mois & 03-août & 31-déc. \\
\hline & Infirmière C & 8 & $50 \%$ & 4 mois & 04-mai & 30-sept. \\
\hline & Infirmière D & 7,5 & $100 \%$ & 8 mois & 01-janv. & 13-août \\
\hline & Infirmière E & 3 & $100 \%$ & 3 mois & 28-sept. & 31-déc. \\
\hline & Infirmière $\mathrm{F}$ & 1 & $50 \%$ & 1 mois & 25-nov. & 31-déc. \\
\hline Total en mois ETP & & & (I) & 31 mois & & \\
\hline
\end{tabular}

Ceci qui représente, une fois déduits les congés et les jours fériés chômés, 4 065h disponible pour la coordination des soins (cf tableau 2).

Tableau 2: Temps de travail effectif des infirmières pour 2009

\begin{tabular}{|c|c|c|}
\hline \multicolumn{3}{|l|}{ Temps de travail 2009} \\
\hline $\begin{array}{l}\text { Total de mois de travail E } \\
\text { Horaire hebdo } \\
\text { Année } \\
\text { Congés } \\
\text { Jours fériés } \\
\text { soit } \\
\text { Semaine travaillées (III) }\end{array}$ & $\begin{array}{l}P \\
52 \text { semaines } \\
5 \text { semaines } \\
10 \mathrm{~J} \\
2 \text { semaines } \\
45 \text { semaines (IV) }=(\mathrm{II}) \times(\mathrm{III})\end{array}$ & $\begin{array}{r}31 \text { mois } \\
35 \mathrm{~h} / \text { semaine }\end{array}$ \\
\hline
\end{tabular}

Ce temps de coordination a permis de prendre en charge 547 patients tous soins confondus, dont 69 qui ont reçu en tout 532 chimiothérapies à domicile, soit une moyenne de 7,7 chimiothérapies par patient. 
Le bilan financier fait état d'un total de dépenses (comptables) de fonctionnement de 489466 $€$. La permanence médicale oncologique, ainsi que les locaux, qui sont actuellement fourni gracieusement par le Centre Léon Bérard, constituent des charges supplétives de $22708 €$ qu'il convient d'ajouter. (cf tableau 3)

Tableau 3: dépenses du réseau Source en 2009

\begin{tabular}{|lr|}
\hline Dépenses du réseau en $\mathbf{2 0 0 9}$ & 2009 \\
\hline source : rapport financier 2009 & $194135 €$ \\
Charges externes & $11703 €$ \\
Impôts et taxes & $198846 €$ \\
Salaires et traitement & $80846 €$ \\
Charges sociales & $3936 €$ \\
Dotations aux amortissements & $\mathbf{4 8 9} 466 €$ \\
Total des dépenses comptables & \\
Eléments mis a disposition & $14769 €$ Supplétif \\
Astreinte médicale oncologue & $7939 €$ Supplétif \\
Mise à diposition locaux & \\
& \\
TOTAL CHARGES DE FONCTIONNEMENT (A) & $512174 €$ \\
\hline
\end{tabular}

Sur ces dépenses, une première somme (49 $864 €$ ) peut être directement affectée aux chimiothérapies: le transport des médicaments (assuré uniquement pour molécules de chimiothérapies, les autres médicaments étant fournis par la pharmacie de proximité du patient qui va les y récupérer lui-même) et la fabrication de la molécule de chimiothérapie.

Tableau 4 : Charges imputables directement aux chimiothérapies

\begin{tabular}{|lr|}
\hline Livraison par LVL & $25794 €$ \\
Salaire chauffeur pour 4 mois & $3565 €$ \\
Location voiture & $746 €$ \\
Carburant & $745 €$ \\
Péage & $311 €$ \\
Préparations chimiothérapies & $18703 €$ \\
Total des charges imputables uniquement aux & \\
chimiothérapies (B) & $49864 €$ \\
\hline
\end{tabular}

Une seconde somme (72 $683 €)$ peut être considérée comme une charge à affecter à une sousactivité. La structure de direction du réseau est calibrée pour supporter une montée en charge et la direction du réseau estime que ce niveau de charge devrait supporter une activité double de celle de 2009.

Tableau 5: Charges soumise à correction d'imputation rationnelle

\begin{tabular}{|c|c|c|c|c|}
\hline \multicolumn{5}{|c|}{ source : rapport financier et comptabilité du réseau } \\
\hline Direction $\quad 53766,00 €$ & $43,5 \%$ & $77154,21 €$ & $50 \%$ & $38577,11 €$ \\
\hline Voiture de fonction & & $7414,10 €$ & $50 \%$ & $3707,05 €$ \\
\hline Medecin coordonateı & $43,5 \%$ & $52859,66 €$ & $50 \%$ & $26429,83 €$ \\
\hline Mise à disposition loc & & $7939,00 €$ & $50 \%$ & $3969,50 €$ \\
\hline Charges associée à la sous-activité (C) & & $145366,97 €$ & $50 \%$ & $72683,49 €$ \\
\hline
\end{tabular}


Aussi avons-nous utilisé, pour les charges de structures qui répondaient à ce critère,un coefficient d'imputation rationnelle de 0,5 .

Tableau 6: Coût d'une heure de coordination

\begin{tabular}{|lr|}
\hline Total des dépenses comptables & $489466 €$ \\
Charges directement liées aux & \\
chimiothérapies & $-49864 €$ \\
Charges de direction soumise à & \\
imputation rationelle & $-72683 €$ \\
Charges supplétives & $22708 €$ \\
Total des charges à répartir sur & $389627 €$ \\
|'ensemble des soins (A) & $4065 \mathrm{~h}$ \\
Heures de coordination infirmière (B) & $95,85 €$ \\
Coût d'une heure de coordination (A)/(B)
\end{tabular}

L'heure de coordination infirmière a donc été " produite » à un coût complet de pleine activité de $95,85 €$.

\subsubsection{Les projections d'activité de coordination pour 2010}

La montée en charge se poursuit en 2010. Un effectif total de 4 infirmières, dont certaines a temps partiel, donne une disponibilité totale de 41 mois équivalent temps plein (cf tableau 7)

Tableau 7 : Composition de l'effectif des infirmières de coordination en 2010

\begin{tabular}{|lrrrr|}
\hline Effectifs payés sur 2010 & & & \\
Infirmière & Infirmière A & 12 & $91,43 \%$ & 11 mois \\
& Infirmière B & 12 & $100 \%$ & 12 mois \\
& Infirmière E & 12 & $100 \%$ & 12 mois \\
& Infirmière F & 12 & $50 \%$ & 6 mois \\
& & & & \\
Nombre de personnes & & 41 Total en nombre de mois ETP ('
\end{tabular}

Cet effectif permet de disposer d'un temps de coordination de 5449 h pour 2010 (cf tableau 8).

Tableau 8 : Structure de fonctionnement de l'effectif d'infirmières libérales pour

$$
\text { 2010) }
$$

\begin{tabular}{|lrrr|}
\hline Temps de travail 2010 & & \\
\hline Horaire hebdo & & \\
Année & 52 semaines & & \\
Congés & 5 semaines & & \\
Jours fériés & $7 \mathrm{~J}$ & & \\
soit & 1,4 semaines & & \\
Semaine travaillées $(\mathrm{VII})$ & 45,6 semaines & $(\mathrm{VIII})=(\mathrm{VII}) \times(\mathrm{II})$ & $1596 \mathrm{~h}$ \\
& & & \\
& & & \\
Temps de travail total potentiel en $2010 \quad(\mathrm{IX})=(\mathrm{VI}) / 12 \times(\mathrm{VIII})$ & $549 \mathrm{~h}$ \\
\hline
\end{tabular}


Cet effectif permet une présence moyenne de 2,5 infirmières par jour d'ouverture (cf tableau 9)

\section{Tableau 9: Structure de fonctionnement de l'effectif d'infirmières libérales pour $\underline{2010)}$}

\begin{tabular}{|c|c|}
\hline \multicolumn{2}{|l|}{ Analyse structure du temps de travail 2010} \\
\hline ETP inscrits à l'effectif 2010 & 3,41 \\
\hline Personnes présentes chaque jour & \\
\hline Temps de travail effectif 2010 & $5449 h$ \\
\hline Jours d'ouverture : 5 jours sur 7 , soit $(365-((52 * 2)+7))(X I I)$ & \\
\hline Plage de permanence $\quad 9 h-18 h(-0,5 h$ déjeuner) (XIII) & $8,50 \mathrm{~h}$ \\
\hline Heures de travail pour permanence $(\mathrm{XIV})=(\mathrm{XI}) \times(\mathrm{XII}) \times(\mathrm{XIII})$ & $5397,50 \mathrm{~h}$ \\
\hline Manque potentiellement $\quad($ IX) $-(\mathrm{XIV})$ & \\
\hline
\end{tabular}

Sur la base d'un mix des soins équivalent à 2009 , cela devrait permettre de prendre en charge 724 patients tous soins confondus, dont 91 qui pourront recevoir en tout 704 chimiothérapies à domicile.

Cette hypothèse de constance du mix patient et de proportionnalité des volumes de prise en charge va nous permettre d'estimer le coût complet de coordination d'une séance de chimiothérapie organisée par le réseau.

\subsubsection{Estimation des temps passés en routine pour la coordination des chimiothérapies}

Les infirmières du réseau ont participé à l'élaboration d'un standard des temps passés aux différentes opérations constituant leur temps de travail. Les temps passés ont été associés à différents inducteurs : les patients pris en charge, le processus de gestion des chimiothérapies, les patients pris en charge pour les chimiothérapies, les séances de chimiothérapies. 
Tableau 9: Inventaire des temps passés à affecter aux chimiothérapies

\begin{tabular}{|c|c|c|c|c|c|c|c|}
\hline & & & & & & & \\
\hline & & & & $\begin{array}{c}\begin{array}{c}\text { Pour } \\
\text { I'organiszion } \\
\text { générale des } \\
\text { soins }\end{array} \\
\end{array}$ & $\begin{array}{c}\text { Pour } \\
\text { I'organisation } \\
\text { générale des } \\
\text { chi miothérapies }\end{array}$ & $\begin{array}{l}\text { Par patient } \\
\text { recevant des } \\
\text { chi miothérapie }\end{array}$ & $\begin{array}{l}\text { Par séance de } \\
\text { chi miothérapie }\end{array}$ \\
\hline \multicolumn{2}{|c|}{$\begin{array}{l}\text { Chaque fois qu'un nouveau patient est } \\
\text { pris en charge pour des chi miothérapies } \\
\text { àdomicile }\end{array}$} & $65 \mathrm{mn}$ & $1,08 \mathrm{~h}$ & & & $1,08 \mathrm{~h}$ & \\
\hline \multirow{2}{*}{$\begin{array}{l}\text { réseau, expliquer aux } \\
\begin{array}{l}\text { Corét tutution de léquipe d'IDE } \\
\text { litéraux }\end{array}\end{array}$} & 1 fois & $20 \mathrm{mn}$ & $0,33 \mathrm{~h}$ & & & $0,33 \mathrm{~h}$ & \\
\hline & 1 fois & $15 \mathrm{mn}$ & $0,25 \mathrm{~h}$ & & & $0,25 \mathrm{~h}$ & \\
\hline $\begin{array}{l}\text { Récupération protocole } \\
\text { ordonance }\end{array}$ & 1 fois & $10 \mathrm{mn}$ & $0,17 \mathrm{~h}$ & & & $0,17 \mathrm{~h}$ & \\
\hline \multirow{2}{*}{$\begin{array}{l}\text { Contact phar macie de ville } \\
\text { Commande matériel à } \\
\text { prestataire }\end{array}$} & 1 fois & $10 \mathrm{mn}$ & $0,17 \mathrm{~h}$ & & & $0,17 \mathrm{~h}$ & \\
\hline & 1 fois & $5 \mathrm{mn}$ & $0,08 \mathrm{~h}$ & & & $0,08 \mathrm{~h}$ & \\
\hline \multirow{2}{*}{$\begin{array}{l}\text { Programmation sur le table au } \\
\text { d'organisation des chimio } \\
\text { Achaque séance de chi miot }\end{array}$} & 1 fois & $5 \mathrm{mn}$ & $0,08 \mathrm{~h}$ & & & $0,08 \mathrm{~h}$ & \\
\hline & therapie & $60 \mathrm{mn}$ & $0,33 \mathrm{~h}$ & & & & $0,33 \mathrm{~h}$ \\
\hline \multirow{2}{*}{$\begin{array}{l}\text { Récupération feu vert } \\
\text { Go informatique, Appel } \\
\text { infirmière, patient, fiche de } \\
\text { transport }\end{array}$} & $\begin{array}{l}\text { chaque fois. } \\
\text { tous sauf } \\
\text { herceptine }\end{array}$ & $10 \mathrm{mn}$ & $0.17 \mathrm{~h}$ & & & & $0,17 \mathrm{~h}$ \\
\hline & chaque fois & $10 \mathrm{mn}$ & $0,17 \mathrm{~h}$ & & & & $0,17 \mathrm{~h}$ \\
\hline \multicolumn{2}{|c|}{$\begin{array}{l}\text { A chaque incident pendant la séance, } \\
\text { avec une fréquence en } 2009 \text { de } 10 \\
\text { incident pour } 532 \text { chi miothéræpies, soit } \\
\text { pour } 2010,10532^{*} 704=13,23 \text { incidents }\end{array}$} & $20 \mathrm{mn}$ & $0,33 \mathrm{~h}$ & & $4,41 \mathrm{~h}$ & & \\
\hline Gestion incidents & 2009 pour & $20 \mathrm{~min}$ & $0,33 \mathrm{~h}$ & & $4,41 \mathrm{~h}$ & & \\
\hline \multicolumn{2}{|c|}{$\begin{array}{l}\text { Chaque semaine, pour l'organi stion } \\
\text { générale des chi miothérapies }\end{array}$} & $40 \mathrm{mn}$ & $0,67 \mathrm{~h}$ & & $34,67 \mathrm{~h}$ & & \\
\hline $\begin{array}{l}\text { Mises à jour du tableau } \\
\text { d'organis ation générale des } \\
\text { chimiothérpies }\end{array}$ & $\begin{array}{l}1 \text { pers } \\
\text { chaque } \\
\text { semaine }\end{array}$ & $20 \mathrm{mn}$ & $0,33 \mathrm{~h}$ & & $17,33 \mathrm{~h}$ & & \\
\hline $\begin{array}{l}\text { Validation des chimio (post } \\
\text { traitement) }\end{array}$ & $\begin{array}{l}\text { pers } \\
\text { chaque } \\
\end{array}$ & $20 \mathrm{mn}$ & $0,33 \mathrm{~h}$ & & $17,33 \mathrm{~h}$ & & \\
\hline \multicolumn{2}{|c|}{$\begin{array}{l}\text { Par an, pour le fonctionnement général } \\
\text { de l'équipe de coordination }\end{array}$} & & $889,08 \mathrm{~h}$ & $889,08 \mathrm{~h}$ & & & \\
\hline $\begin{array}{l}\text { Réunion équipe } \\
\text { hebdomadaire }\end{array}$ & \begin{tabular}{|l} 
tous les \\
présents
\end{tabular} & $60 \mathrm{mn}$ & $1,00 \mathrm{~h}$ & $52,00 \mathrm{~h}$ & & & \\
\hline Prépa de la journée de travail & $\begin{array}{l}\text { chaque } \\
\text { personne } \\
\text { présente }\end{array}$ & $30 \mathrm{mn}$ & $0,50 \mathrm{~h}$ & $317,50 \mathrm{~h}$ & & & \\
\hline Relève SAD & $\begin{array}{l}1 \text { personne } \\
\text { chaque jour }\end{array}$ & $30 \mathrm{mn}$ & $0,50 \mathrm{~h}$ & $127,00 \mathrm{~h}$ & & & \\
\hline Echange dinfos sur SAD & $\begin{array}{l}\text { chaque } \\
\text { personne } \\
\text { présente }\end{array}$ & $10 \mathrm{mn}$ & $0.17 \mathrm{~h}$ & $105,83 \mathrm{~h}$ & & & \\
\hline $\begin{array}{l}\text { Autres besoins d'échange } \\
\text { dans la joumée }\end{array}$ & $\begin{array}{l}\text { chaque } \\
\text { personne } \\
\text { présente }\end{array}$ & $15 \mathrm{mn}$ & $0,25 \mathrm{~h}$ & $158,75 \mathrm{~h}$ & & & \\
\hline Formation & \begin{tabular}{|l|}
4 à 5 jours par \\
an par \\
infirmière \\
coordonnatrice \\
\end{tabular} & & $32,00 \mathrm{~h}$ & $128,00 \mathrm{~h}$ & & & \\
\hline \multicolumn{2}{|c|}{ TOTAL PAR CATEGORIE } & & & $889,08 \mathrm{~h}$ & $39,08 \mathrm{~h}$ & $1,08 \mathrm{~h}$ & $0,33 \mathrm{~h}$ \\
\hline \multirow{2}{*}{\multicolumn{2}{|c|}{ Base de la mutualisation }} & & & 724 patients & 704 séances & 7,7 séancelPat & \\
\hline & & & & 7,7 séanceip at & & & \\
\hline \multicolumn{2}{|l|}{ Coefficient de mutualisation } & & & 0,000179 & 0,0014 & 0,1297 & 1,0000 \\
\hline \multicolumn{2}{|c|}{ Temps répartipar chimiothérapie } & & $0,6887 \mathrm{~h}$ & $0,1594 \mathrm{~h}$ & $0,0555 \mathrm{~h}$ & $0,1405 \mathrm{~h}$ & $0,3333 \mathrm{~h}$ \\
\hline
\end{tabular}

La coordination d'une chimiothérapie à domicile en réseau est réalisée à un coût moyen estimé à $66,01 €(0,6887 \mathrm{~h} \times 95,85 €)$. Le transport des chimiothérapies a été estimé, sur la base d'une étude réalisée sur les six premiers mois de fonctionnement du service de livraison du réseau, à 27,72€ par livraison en moyenne (cf tableau 10). 
Tableau 10: Coût de transport des chimiothérapies

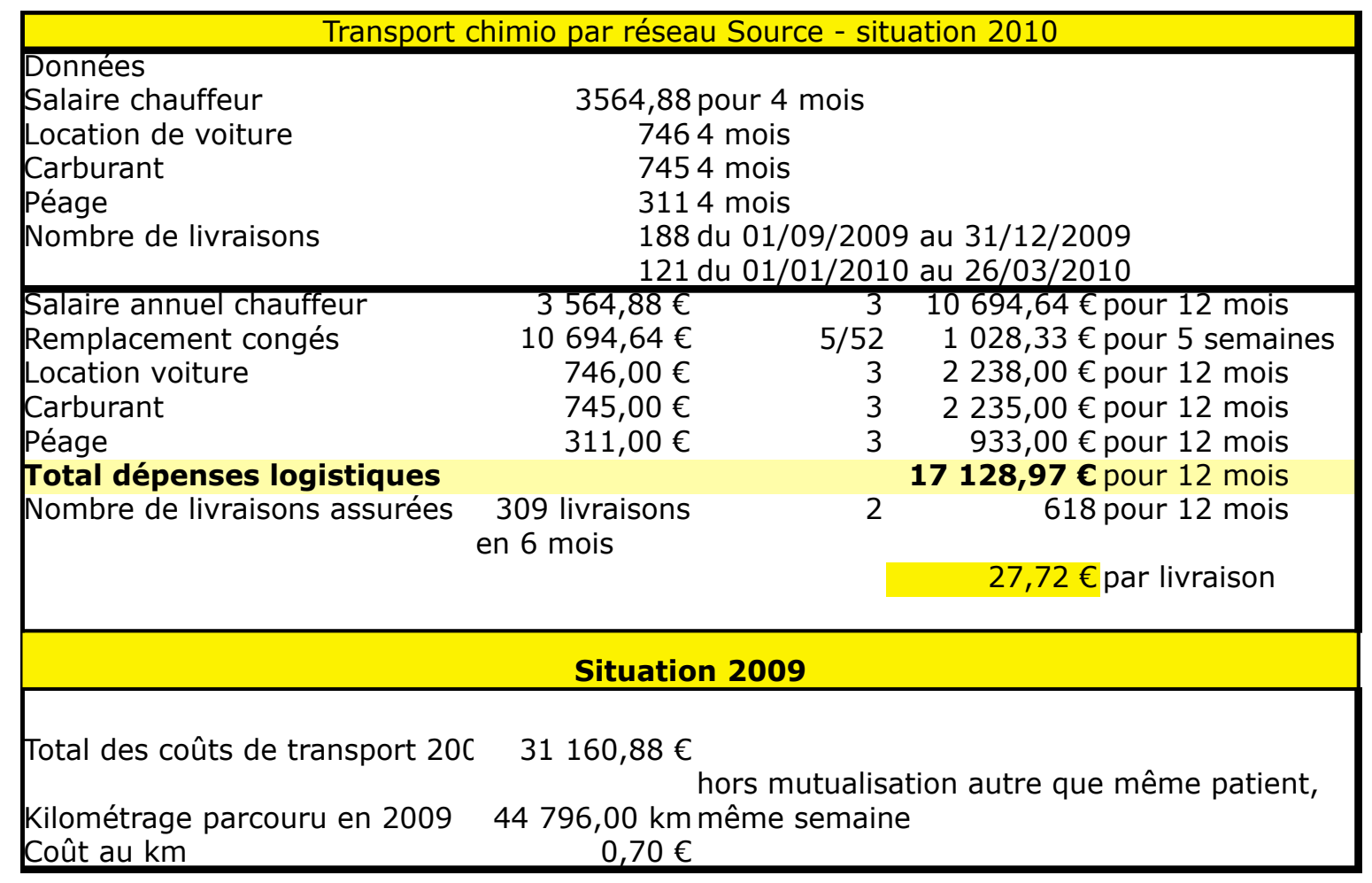

La préparation de chimiothérapie est facturée par la PUI $35 €$ par chimiothérapie. L'injection par l'infirmière varie en fonction de la durée d'administration de la molécule. Le feu vert du médecin est nécessaire quelle que soit la molécule, sauf l'Herceptine.

Tableau 10: Coût complet d'une chimiothérapie réalisée en réseau

\begin{tabular}{|c|c|c|c|}
\hline \multicolumn{4}{|c|}{ Coût complet chimio réseau Source } \\
\hline Coûts de coordination & Chimio courte & $\begin{array}{l}\text { Chimio longue } \\
\text { (1h30 max })\end{array}$ & $\begin{array}{c}\text { Herceptine } \\
\text { (pas de feu } \\
\text { vert } \\
\text { médecin } \\
\text { nécessaire) }\end{array}$ \\
\hline $\begin{array}{l}\text { organisation, coordination, mise à jour } \\
\text { dossier patient } \\
\text { Coûts directs }\end{array}$ & $66,01 €$ & $66,01 €$ & $66,01 €$ \\
\hline 1. Préparation de la chimiothérapie & $35,00 €$ & $35,00 €$ & $35,00 €$ \\
\hline 2. Livraison de la chimiothérapie & $27,72 €$ & $27,72 €$ & $27,72 €$ \\
\hline 3. Feu vert médecin & $23,00 €$ & $23,00 €$ & \\
\hline $\begin{array}{l}\text { 4. Injection par l'infirmière } \\
\text { 5. Déplacement IDE (habite au maximum à }\end{array}$ & $4,73 €$ & $47,25 €$ & $31,50 €$ \\
\hline $10 \mathrm{~km}$ du patient) & $12,30 €$ & $12,30 €$ & $12,30 €$ \\
\hline 6. Kit de perfusion & $15,35 €$ & $15,35 €$ & $15,35 €$ \\
\hline COUT TOTAL POUR UNE CHIMIO & $184,11 €$ & $226,63 €$ & $187,88 €$ \\
\hline
\end{tabular}

Le coût complet d'une chimiothérapie pour l'assurance maladie varie donc, selon notre estimation, de $184,11 €$ à $226,63 €$ hors molécule. 


\section{Le dispositif de facturation des prestations hospitalières}

Les différentes modalités de tarification des quatre solutions existantes pour l'administration des chimiothérapies est présenté dans le tableau 11. Les tarifs hospitaliers retenus sont les tarifs de référence nationaux pour la T2A 2010. Certains ajustements sont spécifiquement apportés comme le correcteur géographique, particulièrement en région parisienne. Cependant, notre réseau comparateur est un réseau lyonnais. Il peut donc être comparé au tarif de référence national.

Tableau 11 : structure de tarification des chimiothérapies

\begin{tabular}{|c|c|c|c|c|c|}
\hline $\begin{array}{l}\text { Mode d'organisation de } \\
\text { hprise en cliarge }\end{array}$ & $\begin{array}{c}\text { Structure tarifaire visble pour l'assurance } \\
\text { mahdie }\end{array}$ & \multicolumn{2}{|c|}{$\begin{array}{l}\text { Totalde la } \\
\text { prestation }\end{array}$} & \multicolumn{2}{|c|}{$\begin{array}{c}\text { Total de la } \\
\text { prestation avec } \\
\text { analyse de saug si } \\
\text { non inc huse dans le } \\
\text { totalprécédent }\end{array}$} \\
\hline $\begin{array}{l}\text { A. Structure hospitalière } \\
\text { type CHU, CH, Hôpital de } \\
\text { cancérologie }\end{array}$ & $\begin{array}{l}\text { T2A } 2010: 379,14 € \text { la séance en ambula toire } \\
\text { (hôpital de jour) }\end{array}$ & $379,14 €$ & $379,14 €$ & $405,60 €$ & $448,80 €$ \\
\hline $\begin{array}{l}\text { B. Structure hospitaliè re } \\
\text { privée (clinique) } \\
\text { (proximité du patient de } \\
30 \mathrm{~km} \text { ) }\end{array}$ & $\begin{array}{l}\text { T2A } 2010 \text { : 286,67€+éventuellement des } \\
\text { honoraires médicaux de } 23 € \text { pour une } \\
\text { consultation de spécialiste }\end{array}$ & $286,67 €$ & $309,67 €$ & $313,13 €$ & $356,33 €$ \\
\hline C. $\mathrm{HAD}$ & $\begin{array}{l}\text { Pour une prise en charge pour ss ul motif de } \\
\text { chimiothérapie, théoriquement, } 181,28 € \text { pour } \\
\text { une joumée sur la base d'un patient avec un IK } \\
\text { de } 70 \text { (moyenne des IK des patients en } \\
\text { chimiothérapie à domicile). Durée moyenne } \\
\text { des séjours en HAD pour seul motif } \\
\text { d'hospitalisation de } 3,8 \text { jours. }\end{array}$ & $181,28 €$ & $688,86 €$ & $362,56 €$ & $688,86 €$ \\
\hline $\begin{array}{l}\text { D. Domicile coordonné } \\
\text { par un rése au te nitorial de } \\
\text { cancérologie }\end{array}$ & $\begin{array}{l}\text { de } 174,38 € \text { à } 228,38 € \text { pour l' administration et } \\
\text { coordination hors moléc ule, pour un patient } \\
\text { habitant à moins de } 10 \mathrm{~km} \text { de l'infímière }\end{array}$ & $174,38 €$ & $228,38 €$ & $200,84 €$ & $298,04 €$ \\
\hline
\end{tabular}

A l'hôpital, la tarification du séjour est valable pour toutes les chimiothérapies réalisées en hôpital de jour, quelle que soit leur durée, et rémunère également les autres soins et examens qui peuvent être réalisés concomitamment. De nombreuses molécules (mais pas toutes) sont facturées en sus (molécules dites sur la liste en sus). La plupart du temps, l'analyse de sang est faite en ville par le patient et facturée en plus à l'assurance maladie. Il arrive cependant dans certains cas qu'elle soit réalisée lors de l'arrivée à l'hôpital.

Le cas de l'Hospitalisation à Domicile est assez particulier et l'analyse difficile en raison de l'absence de détails sur les actes pratiqués. Sur la base des statistiques PMSI 2009 les séjours 
pour seul motif de chimiothérapie ont une moyenne de 3,8 jours, ce qui représente une tarification moyenne assez élevée. La tarification inclut tout autres soins réalisés et tous les médicaments délivrés pendant le séjour. Suivant les HAD, la prise de sang est incluse (le séjour commence la veille et une infirmièreest envoyée au domicile du patient pour effectuer la prise de sang). D'autres ne l'inclus pas, (le patient va au laboratoire, l'HAD n'hospitalise qu'une journée et n'intervient qu'un jour pour la chimiothérapie). Les cures de plusieurs injections sur plusieurs journées successives vont sans doute générer des hospitalisations à domicile en continues sur toute la durée de la cure.

La longueur des hospitalisations à domicile pour seul motif de chimiothérapie peut également laisser penser que ce ne sont pas les mêmes profils de patients que ceux qui sont pris en charge dans les réseaux ou dans les hôpitaux de jour. Ceci est assez logique, puisque les HAD ont été crées pour représenter une alternative aux hospitalisations longues, et pas aux hospitalisations de jour. Cependant, la chimiothérapie à domicile ne pouvant être effectuée que dans le cadre d'un réseau de soins ou d'une HAD, ces dernières interviennent par défaut en l'absence de réseau, ces derniers étant peu nombreux sur le territoire. Elles devraient normalement dans l'avenir leur laisser la place pour tous les cas ou la chimiothérapie ne nécessite pas une hospitalisation de plusieurs jours et conserver la réalisation des chimiothérapies au sein des prises en charge motivées par d'autres considérations.

La tarification est d'ailleurs parfaitement inadaptée pour ce type de soins. Actuellement, une journée d'hospitalisation en HAD pour seule motif de chimiothérapie, pour un patient parfaitement valide, pourrait être facturée en 2010 à 149,33€, soit moins que ce que coûte la plus courte des chimiothérapie en réseau. Or l'HAD est un établissement de santé, avec une structure de fonctionnement plus lourde qu'un réseau. Nous ne conserverons donc pas l'HAD comme une modalités organisationnelle à comparer au réseau de soins.

La comparaison des structures tarifaires existant ne permet pas de se prononcer en raison de l'hétérogénéité des prestations qui sont incluses dans les tarifications hospitalières. Il est possible d'approcher des éléments de comparaison plus pertinents en utilisant l'échelle nationale des coûts hospitaliers 2007 publié sur le site de l'ATIH.

\subsection{Analyse des prestations associées aux facturations}

Pour rendre les données comparables, nous avons utilisé l'Echelle Nationale de Coût 2007 mise en ligne par l'ATIH.

\subsubsection{Les coûts associés aux séjours hospitaliers}

L'Echelle Nationale de coûts disponible sur le site de l'Agence Technique de l'Information Hospitalière présente une estimation des coûts des séjours hospitaliers par groupe homogène de malade. L'Etude Nationale des Coûts a été constituée à partir des données de coûts collectées par 55 établissements ex-DGF et 44 établissements ex-OQN et jugées de qualité suffisante.

Le coût complet est obtenu après plusieurs corrections qui permettent d'homogénéiser les conditions de calcul nationales : (1) abattements liés aux MERRI (mission d'enseignement et de recherche) qui conduisent de fait à allouer entre 9 et $17 \%$ de ressources supplémentaires à certains établissements (2) neutralisation du coefficient géographique, qui conduit à allouer plus de ressources à certaines zones (environs $7 \%$ en île de France) (3) élimination des séjours extrêmes en durée et en coût 
Tableau 12: Analyse de 1'Echelle Nationale de coûts pour les séjours hospitaliers

\begin{tabular}{|c|c|c|c|c|c|}
\hline Personnel autre clinique (hors SI SC réa) & $24,77 €$ & $4,93 €$ & $\mathrm{X}$ & & \multirow{3}{*}{$\begin{array}{l}\text { avant comparaison pour estımer la part de la seule } \\
\text { chimiothérapie. Dans le cadre d'un réseau de soins, toute autre } \\
\text { prestation sera tarifée à part et représentera un coût } \\
\text { supplémentaire. }\end{array}$} \\
\hline Personnel médical clinique (hors SI SC réa) & $32,38 €$ & $0,00 €$ & $\mathrm{X}$ & & \\
\hline Personnel soignant clinique (hors SI SC réa) & $73,34 €$ & $32,07 €$ & $\mathrm{X}$ & & \\
\hline Dépenses de Surveillance Continue & $0,06 €$ & $0,00 €$ & & $\mathrm{X}$ & \multirow{3}{*}{$\begin{array}{l}\text { Le transfert d'un patient en réanimation ou dans un service de } \\
\text { soins intensif serait facturé en sus pour une prise en charge en } \\
\text { réseau. }\end{array}$} \\
\hline Dépenses Soins Intensifs & $0,10 €$ & $0,00 €$ & & $\bar{X}$ & \\
\hline Dépenses Réanimation & $0,00 €$ & $0,00 €$ & & $\mathrm{X}$ & \\
\hline Dépenses totales Anesthésie & $0,23 €$ & $0,00 €$ & & $\mathrm{X}$ & \multirow{10}{*}{$\begin{array}{l}\text { Les dépenses correspondantes attestent de la réalisation d'autres } \\
\text { actes que la chimiothérapie, qui dans le cadre du réseau seraient } \\
\text { facturées en sus. Elles sont donc exclues pour la comparaison. }\end{array}$} \\
\hline Dépenses totales Bloc & $1,22 €$ & $0,10 €$ & & $\mathrm{X}$ & \\
\hline Dépenses totales Bloc Obstétrique & $0,00 €$ & $0,00 €$ & & $\mathrm{X}$ & \\
\hline Dépenses totales Dialyse & $0,00 €$ & $0,00 €$ & & $\bar{X}$ & \\
\hline Dépenses totales Exploration fonctionnelle & $1,28 €$ & $0,00 €$ & & $\mathrm{X}$ & \\
\hline Dépenses totales Imagerie & $5,18 €$ & $0,01 €$ & & $\bar{X}$ & \\
\hline Dépenses totales Imagerie interventionnelle & $0,04 €$ & $0,00 €$ & & $\mathrm{X}$ & \\
\hline Dépenses totales Laboratoire & $6,39 €$ & $0,00 €$ & & $\mathrm{X}$ & \\
\hline Dépenses totales Laboratoire Hors nomenclature & $1,25 €$ & $0,00 €$ & & $\mathrm{X}$ & \\
\hline Dépenses totales Radiothérapie & $1,60 €$ & $0,00 €$ & & $\mathrm{X}$ & \\
\hline Dépenses totales SMUR terrestre & $0,03 €$ & $0,00 €$ & & $\mathrm{X}$ & \multirow{3}{*}{$\begin{array}{l}\text { Les dépenses de SMUR et d'Urgence représenteraient pour le } \\
\text { réseau, l'utilisation de structures de repli. Elles sont exclus de } \\
\text { notre comparaison. }\end{array}$} \\
\hline Dépenses totales SMUR aérien & $0,00 €$ & $0,00 €$ & & $\mathrm{X}$ & \\
\hline Dépenses totales Urgences & $0,01 €$ & $0,01 €$ & & $\bar{X}$ & \\
\hline Dépenses totales Autres Medico-technique & $0,42 €$ & $0,00 €$ & & $\mathrm{X}$ & Seraient facturées en sus dans la prise en charge en réseau \\
\hline L GG (Logistique Générale) Blanchisserie & $3,20 €$ & $2,88 €$ & $\mathrm{X}$ & & \multirow{9}{*}{$\begin{array}{l}\text { Une partie des coûts de logistique générale et médicale sont } \\
\text { déclenchés par d'autres soins que la chimiothérapie, aussi } \\
\text { conviendrait-il de retraiter ce poste avant comparaison pour } \\
\text { estimer la part de la seule chimiothérapie. Dans le cadre d'un } \\
\text { réseau de soins, toute autre prestation sera tarifée à part et } \\
\text { représentera un coût supplémentaire. }\end{array}$} \\
\hline LGG Restauration & $4,85 €$ & $4,41 €$ & $\mathrm{X}$ & & \\
\hline LGG Accueil et gestion des malades & $5,02 €$ & $6,32 €$ & $\bar{X}$ & & \\
\hline LGG Services administratifs à caractère général & $24,77 €$ & $25,68 €$ & $\mathrm{X}$ & & \\
\hline LGG Services administratifs liés au personnel & $10,87 €$ & $1,44 €$ & $\mathrm{X}$ & & \\
\hline LGG DIM & $3,04 €$ & $1,78 €$ & $\mathrm{X}$ & & \\
\hline LGG DSIO & $7,43 €$ & $2,69 €$ & $\bar{X}$ & & \\
\hline LGG Entretien maintenance & $9,91 €$ & $7,95 €$ & $\bar{X}$ & & \\
\hline LGG Services hôteliers & $17,01 €$ & $9,57 €$ & $\bar{X}$ & & \\
\hline $\begin{array}{l}\text { LGG Brancardage et transport pedestre des } \\
\text { patients }\end{array}$ & $2,50 €$ & $1,09 €$ & & $\mathrm{X}$ & \multirow{2}{*}{$\begin{array}{l}\text { La moyenne des Indices de Karnosky en réseau est de } 80 \text {. Le } \\
\text { patient est donc autonome. Toute autre examen nécessitant } \\
\text { déplacement serait facturé en sus. }\end{array}$} \\
\hline LGG Transport motorisé des patients (hors SMUR) & $1,72 €$ & $0,00 €$ & & $\mathrm{X}$ & \\
\hline LM (Logistique Médicale) Pharmacie & $49,09 €$ & $26,72 €$ & $\mathrm{X}$ & & \multirow{6}{*}{$\begin{array}{l}\text { Une partie des coûts correspondants peuvent être générée par } \\
\text { d'autres soins pris en charge en même temps que la } \\
\text { chimiothérapie, aussi conviendrait-il de retraiter les coûts pour } \\
\text { en tenir compte. }\end{array}$} \\
\hline LM Stérilisation & $0,76 €$ & $0,04 €$ & $\mathrm{X}$ & & \\
\hline LM Génie Biomédical & $0,46 €$ & $0,08 €$ & $\mathrm{X}$ & & \\
\hline LM Hygiène et Vigilance & $7,36 €$ & $1,88 €$ & $\bar{X}$ & & \\
\hline Autre LM & $0,80 €$ & $0,00 €$ & $\bar{X}$ & & \\
\hline Coûts Directs (CD) Autres consommables & $12,82 €$ & $7,95 €$ & $\bar{X}$ & & \\
\hline CD DMI non facturables en sus & $0,84 €$ & $0,19 €$ & & $\mathrm{X}$ & \multirow{4}{*}{ Seraient facturées en sus dans la prise en charge en réseau } \\
\hline CD DMI facturables en sus & $0,20 €$ & $0,05 €$ & & $\mathrm{X}$ & \\
\hline CD Médicaments ATU & $5,68 €$ & $0,00 €$ & & $\bar{X}$ & \\
\hline CD Sang & $6,49 €$ & $0,93 €$ & & $\bar{X}$ & \\
\hline $\begin{array}{l}\text { CD Spécialités pharmaceutiques non facturables en } \\
\text { sus }\end{array}$ & $24,16 €$ & $22,32 €$ & & $\mathrm{X}$ & \multirow{2}{*}{$\begin{array}{l}\text { Toutes les molécules seraient facturées en sus dans la prise en } \\
\text { charge en réseau }\end{array}$} \\
\hline CD Spécialités pharmaceutiques facturables en sus & $668,41 €$ & $721,07 €$ & & $\mathrm{X}$ & \\
\hline CD Sous-traitance SMUR & $0,03 €$ & $0,00 €$ & & $\bar{X}$ & Non pris en compte en l'état (voir SMUR ci-avant) \\
\hline CD Sous-traitance Imagerie & $0,26 €$ & $0,00 €$ & & $\bar{X}$ & \multirow{13}{*}{ Seraient facturées en sus dans la prise en charge en réseau } \\
\hline CD Sous-traitance Laboratoire & $2,19 €$ & $0,00 €$ & & $\bar{X}$ & \\
\hline CD Sous-traitance Laboratoire Hors nomenclature & $0,62 €$ & $0,00 €$ & & $\bar{X}$ & \\
\hline CD Sous-traitance Hospitalisation à l'extérieur & $0,01 €$ & $0,09 €$ & & $\bar{X}$ & \\
\hline CD Sous-traitance Autre & $0,68 €$ & $2,11 €$ & & $\mathrm{X}$ & \\
\hline CD Honoraires des $\mathrm{PH}$ & $0,15 €$ & & & $\mathrm{X}$ & \\
\hline CD Rémunération à l'acte du personnel & $0,52 €$ & & & $\mathrm{X}$ & \\
\hline $\begin{array}{l}\text { Honoraires médicaux - Laboratoire d'anatomie } \\
\text { pathologie }\end{array}$ & & $0,01 €$ & & $\mathrm{X}$ & \\
\hline Honoraires médicaux - Laboratoires autres & & $0,67 €$ & & $\mathrm{X}$ & \\
\hline Honoraires médicaux - Imagerie & & $0,33 €$ & & $\mathrm{X}$ & \\
\hline Honoraires médicaux - Anesthésie & & $0,00 €$ & & $\mathrm{X}$ & \\
\hline Honoraires médicaux - Obstétrique & & $0,00 €$ & & $\mathrm{X}$ & \\
\hline Honoraires médicaux - Chirurgie & & $0,02 €$ & & $\mathrm{X}$ & \\
\hline Honoraires médicaux - Autres actes médicaux & & $29,94 €$ & $\mathrm{X}$ & & $\begin{array}{l}\text { Pris en compte à hauteur de } 23 € \text { identifié dans la structure } \\
\text { tarifaire }\end{array}$ \\
\hline Honoraires soignants & & $0,02 €$ & & $\mathrm{X}$ & \multirow{2}{*}{ Seraient facturées en sus dans la prise en charge en réseau } \\
\hline Honoraires autres & & $0,00 €$ & & $\mathrm{X}$ & \\
\hline Structure Immobilier & $7,12 €$ & $2,79 €$ & $\mathrm{X}$ & & \multirow{2}{*}{$\begin{array}{l}\text { Pris en compte au titre des coûts immobiliers et des besoins } \\
\text { financiers liés, pour partie liés aux autres soins prodigués dans } \\
\text { les mêmes séjours. }\end{array}$} \\
\hline Structure financier & $16,97 €$ & $28,79 €$ & $\mathrm{X}$ & & \\
\hline
\end{tabular}


Lorsque le nombre de séjour est supérieur à 30, le coût moyen affiché est la moyenne des établissements sélectionnés. Le coût complet peut varier d'un établissement à l'autre en fonction des techniques employées. Le calcul d'un intervalle de confiance permet de situer que le coût réel a statistiquement $95 \%$ de chance de se trouver dans l'intervalle entre une borne haute et une borne basse ce qui permet de donner une indication sur la précision de la moyenne ainsi calculée. Dans le cas des séjours ambulatoires de chimiothérapie que nous étudions, le nombre de séjours analysés : 207766 pour le secteur public et PSPH (20,43\% des séjours) et 89721 pour le secteur privé (12,69\% des séjours). La moyenne du coût du GHM pour le secteur ex DGF se situe à $1021,70 €$ avec un intervalle de confiance situé entre $969,68 €$ et $1073,70 €$. Soit une variation de 5\% autour du coût moyen. Cette estimation est donc assez précise. La moyenne du coût du GHM pour le secteur ex OQN se situe à 886,17€ avec un intervalle de confiance situé entre $658,71 €$ et 1 182,56 $€$. Soit une variation de $33,45 \%$ autour du coût moyen. Cette estimation est donc beaucoup moins précise.

Le coût complet est présenté hors frais de structure, défini dans l'ENC en deux postes de dépenses. D'une part les frais immobiliers sont constitués par les charges liées au patrimoine immobilier des établissements : charges locatives et de copropriétés, entretien et réparation des biens immobiliers, taxe foncière et autres impôts locaux, dotations aux amortissements des constructions, des agencements et aménagements de terrains. D'autre part les frais financiers constitués par les charges financières incorporables dans l'ENC : intérêts des emprunts et dettes, intérêts des crédits bail retraités et intérêts des comptes courants créditeurs.

Dans la perspective de la comparaison des coûts de réalisation de la chimiothérapie en Etablissement de Santé versus un réseau de santé, il convient de retraiter cette base des coûts hospitaliers. Certaines charges sont liées à des prestations qui, si elles devaient être organisées par le réseau de santé, viendrait augmenter les coûts de la prise en charge. Nous allons donc détailler le retraitement poste par poste et les raisons de nos choix dans le tableau ci-après.

Après avoir déterminé quels étaient les postes de dépenses qui permettaient de rendre comparables la chimiothérapie réalisée en réseau et la même prestation réalisée en hôpital de jour, il nous a fallu estimer ce que pouvait représenter les autres soins dans cette base de coûts. Nous nous sommes livrés à une approximation à partir des données disponibles sur les bases de l'ATIH. Nous avons utilisé le dénombrement de l'intégralité des actes enregistrés dans les séjours de la base pour l'année 2007, année qui a servi de base à l'ENC, et qui n'étaient pas des injections de produits anticancéreux. Les actes enregistrés ne sont sans doute pas exhaustif, mais cela donne une première approche. Nous avons arbitrairement considéré le ratio entre actes autres que chimiothérapie et nombre de séjours $(13,80 \%$ pour le public et $2,69 \%$ pour le privé) pour corriger les postes de charge impactés par la présence d'autres actes dans le séjour.

Tableau 13: Estimation corrigée du coût de la chimiothérapie en Etablissement Hospitalier

\begin{tabular}{|c|c|c|c|c|c|c|}
\hline \multirow{2}{*}{ Postes de coîts coût complet } & \multirow{2}{*}{$\begin{array}{l}\text { Fubric et } \\
\text { FSPH }\end{array}$} & \multirow{2}{*}{ Frivé } & \multicolumn{2}{|c|}{$\begin{array}{l}\text { Coefificient de } \\
\text { prise en canqte }\end{array}$} & \multirow{2}{*}{$\begin{array}{c}\text { Fublic et } \\
\text { PSYH }\end{array}$} & \multirow{2}{*}{ Frivé } \\
\hline & & & $\begin{array}{l}\text { Fublic } \\
\text { et PSPY }\end{array}$ & Frivé & & \\
\hline 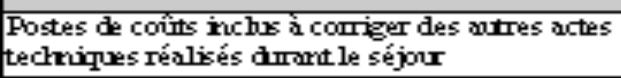 & $313,54 \mathrm{E}$ & $109, \pi 9 \notin$ & $8169 \%$ & $97,10 \%$ & $256,13 \mathrm{E}$ & $164,87 €$ \\
\hline Hanarires méticanx - Antres actes méticanx & & $2994 €$ & & $23,00 €$ & & $23,00 €$ \\
\hline \multicolumn{5}{|l|}{ Tatoldes coûts retraités } & $256,13 €$ & $187,87 €$ \\
\hline
\end{tabular}


La différence entre les coûts retraités structure publique et structure privée est plus faible que lorsque l'on compare les tarifs, mais elle reste cependant significative (Tableau 14).

$\underline{\text { Tableau } 14 \text { : Coût comparé de l'injection d'une chimiothérapie, hors molécule }}$

\begin{tabular}{|lcc|}
\hline Réseau de soins & de $184,11 €$ à $226,63 €$ pour une durée maximum d'1,30 heure \\
\hline Hôpital de jour public & $256,13 €$ & toute durée \\
\hline Hôpital de jour privé & $187,87 €$ & toute durée \\
\hline
\end{tabular}

Il convient pourtant de ne pas trop s'y attarder sans études plus approfondies. Les structures hospitalières accueillent par ailleurs des chimiothérapies de durées très variables, qui vont de l'injection simple à la perfusion de plusieurs heures, alors que celles réalisées en réseau ne dépassent pas une heure trente. Les CHU et les CLCC qui accueillent souvent les patients les plus complexes à traiter ont sans doute des durées plus longues que les structures privées, mais nous ne disposons pas des données nécessaires pour en estimer l'impact.

Cette estimation permet cependant de donner du sens aux comparaisons en mettant en perspective des éléments plus comparables que les données brutes. Les fourchettes de coûts sont proches et compte tenu des hypothèses et remarques formulés, les éléments analysés ne permettent pas de discriminer un mode d'administration sur la base de son coût de fonctionnement. Bien que cela ne soit pas parfaitement démontré, il semblerait qu'à soins et durée égale, les coûts d'administration soient relativement proches.

Il reste cependant un élément non négligeable du coût des chimiothérapies pour être complet sur l'analyse comparée, celui du transport des patients.

\section{Le problème particulier du transport des patients}

Le coût de transport des patients est une donnée extrêmement variable en fonction de l'éloignement de ces derniers du lieu où la chimiothérapie doit leur être administrée, et du moyen de transport qu'ils vont utiliser. Ils peuvent bénéficier d'un transport sanitaire, qui sera pris en charge par la sécurité sociale lorsqu'il est prescrit par un médecin. Le cancer est considéré comme une affection longue maladie, et les patients atteints de cancer sont pris en charge à $100 \%$. Les centres de traitement des cancers sont des centres urbains, et la cancérologie va être un consommateur important de transports sanitaires pour acheminer les patients jusqu'aux centres de traitement.

Nous allons utiliser la grille de remboursement de 1'assurance maladie pour intégrer les transports à notre raisonnement. Les remboursements admis par la sécurité sociale pour les trois solutions sont présentées dans le tableau 15.

Tableau 15 : Coût de transport des patients, données URCAM

\begin{tabular}{|l|r|r|r|}
\cline { 2 - 4 } \multicolumn{1}{c|}{} & \multicolumn{2}{c|}{ Fixe } & \multicolumn{1}{c|}{ Variable } \\
\hline & $\begin{array}{c}\text { Fixe } \\
\text { minimum }\end{array}$ & $\begin{array}{c}\text { Fixe } \\
\text { maximum }\end{array}$ & $\begin{array}{c}\text { Prix du km } \\
\text { (max) }\end{array}$ \\
\hline Sans prise en charge & $0,00 €$ & $0,00 €$ & $0,35 €$ \\
\hline Voiture personnelle & $11,48 €$ & $13,28 €$ & $0,83 €$ \\
\hline VSL & $51,30 €$ & $57,37 €$ & $2,12 €$ \\
\hline Ambulance & &
\end{tabular}

L'administration de la chimiothérapie nécessite soit que le patient se rende dans un hôpital de 
jour, soit que l'on achemine la (ou les) molécules de chimiothérapie chez lui, pour que l'infirmière libérale de proximité puisse lui les administrer.

Le schéma ci-après présente nos différentes alternatives pour la chimiothérapie ambulatoire, assorties ou non de coûts de transport selon les différentes modalités possibles et en fonction de la distance qui sépare le patient et la structure de soin hospitalière. Pour permettre cette simulation, le coût de transport des chimiothérapies organisées par le réseau, qui avait été calculé de manière forfaitaire a été rendu variable sur la base des kilomètres qui ont été nécessaires à la livraison des patients en 2009. Les dépenses totales se montaient à $31160 €$ pour $44796 \mathrm{~km}$ environ, soit $0,70 €$ par $\mathrm{km}$. Ce coût au kilomètre tient compte de la mutualisation permise par l'organisation de tournée et les livraisons groupées (une livraison par semaine lorsqu'il y a plusieurs chimiothérapies dans la même semaine). Nous n'avons pas retenu d'hypothèse de mutualisation concernant le transport du patient.

\section{Figure 1: Simulation du coût pour l'assurance maladie des solutions organisationnelles en fonction de l'éloignement des patients}

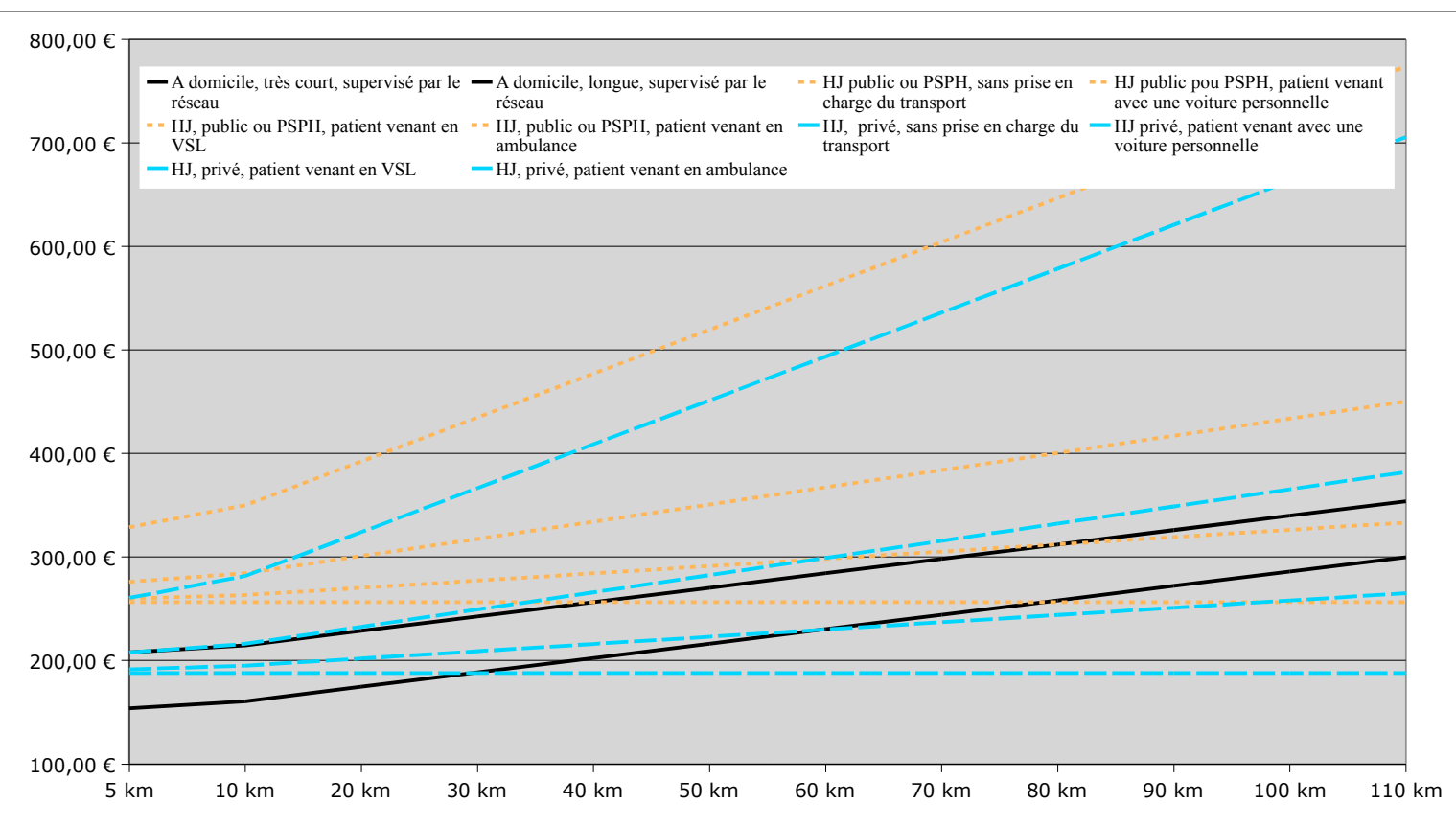

A ce jour, les chimiothérapies étant préparées dans les pharmacies hospitalières, la distance à parcourir par le patient pour aller de chez lui à l'hôpital est la même que la distance que doit parcourir la chimiothérapie pour aller de l'hôpital a chez lui. La figure 1 présente les coûts des différentes alternatives présentées ci-avant, en fonction de la distance entre le patient et la structure de soins, qui à ce jour est également le lieu de préparation de la molécule administrée à domicile.

Cette simulation met en évidence de façon claire l'impact des coûts de transport sur le coût global de la séance de chimiothérapie. Sur notre schéma, on peut par exemple lire que lorsque la séance consiste en une injection et que le patient réside à moins d'environ $30 \mathrm{~km}$ d'une structure privée qui peut le prendre en charge, alors le réseau est moins coûteux. S'il habite à plus de $30 \mathrm{~km}$ et si son déplacement ne fait pas l'objet d'une prise en charge financière, une structure hospitalière privée peut être moins coûteuse. Cette dernière sera moins coûteuse si la chimiothérapie dure 1 heure et demi, que le patient habite à $40 \mathrm{~km}$ vient avec son véhicule personnel, et ce même s'il demande un remboursement kilométrique. Enfin, lorsque le patient 
utilise une ambulance pour recevoir une simple chimiothérapie, le réseau devient bien plus performant que toute autre solution.

\section{Conclusion}

Notre simulation montre la sensibilité de la fonction de coût à un paramètre qui n'est pas médical, mais souligne également qu'il n'existe pas une réponse unique quant à la solution la plus intéressante pour l'assurance maladie. Cette dernière est fonction de l'éloignement du patient, de la durée de la séance, mais également de la possibilité d'organiser le même jour des actes qui, ne pouvant se dérouler à domicile comme la radiothérapie ou les examens de radiologie, mutualiseront le frais de transport.

\section{Bibliographie :}

INCA (2009), Situation de la chimiothérapie des cancers en 2009, publication disponible sur www.ecancer.fr.

Direction générale de la Santé (2003) les 70 mesures du Plan Cancer.

Haute Autorités de Santé (2005) Analyse comparée de la chimiothérapie anticancéreuse administrée à l'hôpital ou prise en charge à domicile: aspects économiques et organisationnels,rapport, Juin.

Réseau territorial Saint Nazaire (Onco Pays de Loire) (2007) Chimiothérapie à domicile, évaluation à 3 ans, document d'évaluation disponible sur internet

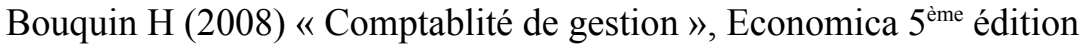


Page 121 sur 122 


\section{RESUME}

Le traitement du cancer requiert l'usage de molécules de chimiothérapie, administrées en plusieurs séquences successives, à ce jour principalement par voie intraveineuse. Il nécessite l'intervention d'un personnel soignant qualifié et est effectué principalement dans les services de jour des hôpitaux et des cliniques qui ont organisé en 2009 près de deux millions de séances.

L'idée de réaliser les chimiothérapies au domicile des patients, administrées par les infirmières libérales de ville, s'est développée dès les années 90 suite à l'effet combiné de l'augmentation des cancers qui saturaient les plateaux techniques de cancérologie et d'une volonté d'externaliser certaines prestations hospitalières pour réduire les coûts de prise en charge. Aujourd'hui, de nombreuses molécules de chimiothérapie peuvent être administrées dans le cadre d'une structure d'Hospitalisation à Domicile (HAD) ou à domicile par des libéraux coordonnés par un réseau de soins. Or, en 2009, la chimiothérapie à domicile représente moins de $1 \%$ de près de 2 millions de séances ambulatoires organisées chaque année en France.

L'objet de ce rapport est de présenter les résultats d'une étude sur l'organisation de la chimiothérapie ambulatoire et de ses alternatives à domicile, que nous avons mené dans le cadre d'un projet portant sur l'Organisation des Soins à Domicile (Projet OSAD, financé par la région Rhône Alpes). Cette étude investigue les données relatives aux coûts des alternatives, mais également les stratégies des différents acteurs de soins au regard de cette prestation.

Cette étude approfondie montre plus une complémentarité des modes d'administration sur le plan économique. La chimiothérapie est compétitive sous certaines conditions. Le lieu de vie du patient et les modes de transports auxquels il doit avoir recours jouent un rôle majeur dans la comparaison des solutions hôpital ou domicile, et plus que le fait d'être à domicile, c'est pouvoir recevoir son traitement à proximité de son domicile qui a un impact sur les coûts de prise en charge. L'étude nous permet également de montrer que les modèles d'activité des hôpitaux freinent le développement de la chimiothérapie à l'extérieur de l'hôpital. 